\title{
Adiabatic theorems with and without spectral gap condition for non-semisimple spectral values
}

\author{
Jochen Schmid \\ Fachbereich Mathematik, Universität Stuttgart, D-70569 Stuttgart, Germany \\ jochen.schmid@mathematik.uni-stuttgart.de
}

\begin{abstract}
We establish adiabatic theorems with and without spectral gap condition for general operators $A(t): D(A(t)) \subset X \rightarrow X$ with possibly time-dependent domains in a Banach space $X$. We first prove adiabatic theorems with uniform and non-uniform spectral gap condition (including a slightly extended adiabatic theorem of higher order). In these adiabatic theorems the considered spectral subsets $\sigma(t)$ have only to be compact - in particular, they need not consist of eigenvalues. We then prove an adiabatic theorem without spectral gap condition for not necessarily (weakly) semisimple eigenvalues: in essence, it is only required there that the considered spectral subsets $\sigma(t)=\{\lambda(t)\}$ consist of eigenvalues $\lambda(t) \in \partial \sigma(A(t))$ and that there exist projections $P(t)$ reducing $A(t)$ such that $\left.A(t)\right|_{P(t) D(A(t))}-\lambda(t)$ is nilpotent and $\left.A(t)\right|_{(1-P(t)) D(A(t))}-\lambda(t)$ is injective with dense range in $(1-P(t)) X$ for almost every $t$. In all these theorems, the regularity conditions imposed on $t \mapsto A(t)$, $\sigma(t), P(t)$ are fairly mild. We explore the strength of the presented adiabatic theorems in numerous examples. And finally, we apply the adiabatic theorems for time-dependent domains to obtain - in a very simple way - adiabatic theorems for operators $A(t)$ defined by symmetric sesquilinear forms.
\end{abstract}

2010 Mathematics Subject Classification: 34E15, 34G10, 35Q41, 47D06

Key words and phrases: adiabatic theorems with and without spectral gap condition for general operators and not necessarily (weakly) semisimple spectral values, adiabatic theorems for operators with time-independent or time-dependent domains

\section{Introduction}

Adiabatic theory for general - as opposed to skew self-adjoint - operators is a quite young area of research which was initiated in [39] and developed further in [2], 25], [8]. It is concerned - in general terms - with densely defined linear operators $A(t): D(A(t)) \subset$ $X \rightarrow X$ in a Banach space $X$ over $\mathbb{C}$, subsets $\sigma(t)$ of $\sigma(A(t))$ and bounded projections $P(t)$ in $X$ (for $t \in I:=[0,1])$ such that 
- $A(t)$ is closed for every $t \in I$ and the initial value problems

$$
x^{\prime}=A(\varepsilon s) x \quad\left(s \in\left[0, \frac{1}{\varepsilon}\right]\right) \quad \text { or } \quad x^{\prime}=\frac{1}{\varepsilon} A(t) x \quad(t \in[0,1])
$$

with initial conditions $x\left(s_{0}\right)=y$ or $x\left(t_{0}\right)=y$ (and initial times $s_{0} \in\left[0, \frac{1}{\varepsilon}\right)$, $\left.t_{0} \in[0,1)\right)$ are well-posed on the spaces $D(A(t))$ for every value of the slowness parameter $\varepsilon \in(0, \infty)$ (Condition 1.1).

- $\sigma(t)$ is a compact subset of $\sigma(A(t))$ for every $t \in I$ (Condition 1.2).

- $P(t)$ commutes with $A(t)$ and $\left.A(t)\right|_{P(t) D(A(t))}$ resp. $\left.A(t)\right|_{(1-P(t)) D(A(t))}$, in some natural sense (specified below), is spectrally related to $\sigma(t)$ as much resp. as little as possible for every $t \in I$ except possibly for some few $t$ (Condition 1.3).

What one wants to know first of all in adiabatic theory is the following: when under which additional conditions on $A, \sigma$ and $P$ - does the evolution $U_{\varepsilon}$ generated by the operators $\frac{1}{\varepsilon} A(t)$ (Condition 1.1) approximately follow the spectral subspaces $P(t) X$ related to the spectral subsets $\sigma(t)$ of $A(t)$ (Condition 1.3) as the slowness parameter $\varepsilon$ tends to 0? Shorter (and more precisely): under which conditions is it true that

$$
(1-P(t)) U_{\varepsilon}(t) P(0) \longrightarrow 0 \quad(\varepsilon \searrow 0)
$$

(with respect to a certain operator topology) for all $t \in I$ ? Adiabatic theorems are, by definition, theorems that give such conditions. And this way of speaking is, etymologically, quite appropriate since the word "adiabatos" simply means "admitting no transitions" and, after all, adiabatic theorems just state that for small $\varepsilon$ there will be almost no more transitions from the space $P(0) X$ to the spaces $(1-P(t)) X$. We distinguish adiabatic theorems with spectral gap condition (uniform or non-uniform) and adiabatic theorems without spectral gap condition depending on whether $\sigma(t)$ is isolated in $\sigma(A(t))$ for every $t \in I$ (uniformly or non-uniformly) or not (Section 2.1).

In this paper, we are going to prove adiabatic theorems with and without spectral gap condition for not necessarily (weakly) semisimple spectral values $\lambda(t) \in \sigma(t)$ of general not necessarily skew self-adjoint - linear operators $A(t)$ with time-independent domains (Section 3 and 4) or time-dependent domains (Section 5 and 6). We thereby carry further the adiabatic theory of [2], 25], [8, for instance, and develop a general and systematic adiabatic theory with a special emphasis on the case without spectral gap. In more detail, the contents of the present paper can be described as follows.

In the adiabatic theorems with (uniform or non-uniform) spectral gap condition (Section 3 and 5.1), the relation between $P(t)$ and $A(t), \sigma(t)$ - vaguely described in Condition 1.3 above - will be assumed to be as follows: for all but countably many $t \in I, P(t)$ is a bounded projection commuting with $A(t)$, such that $P(t) D(A(t))=P(t) X$ and

$$
\sigma\left(\left.A(t)\right|_{P(t) D(A(t))}\right)=\sigma(t) \quad \text { whereas } \quad \sigma\left(\left.A(t)\right|_{(1-P(t)) D(A(t))}\right)=\sigma(A(t)) \backslash \sigma(t) .
$$


(We will, more briefly, call such projections associated with $A(t)$ and $\sigma(t)$.) Such projections always exist in the case with spectral gap and they are uniquely determined by $A(t)$ and $\sigma(t)$. Indeed, they are, of course, just given by

$$
P(t)=\frac{1}{2 \pi i} \int_{\gamma_{t}}(z-A(t))^{-1} d z,
$$

where $\gamma_{t}$ is a cycle in $\rho(A(t))$ with indices $\mathrm{n}\left(\gamma_{t}, \sigma(t)\right)=1$ and $\mathrm{n}\left(\gamma_{t}, \sigma(A(t)) \backslash \sigma(t)\right)=1$ (Riesz projection). In particular, in the special situation where $\sigma(t)=\{\lambda(t)\}$ and $\lambda(t)$ is a pole of the resolvent of $A(t)$, these projections satisfy

$$
P(t) X=\operatorname{ker}(A(t)-\lambda(t))^{k} \quad \text { and } \quad(1-P(t)) X=\operatorname{rg}(A(t)-\lambda(t))^{k}
$$

for all $k \in \mathbb{N}$ with $k \geq m(t)$, where $m(t)$ is the order of the pole. In [39], [2, 25], 8] which, so far, are the only works proving adiabatic theorems with spectral gap for not necessarily skew self-adjoint operators $A(t)$ - the abovementioned special situation of singletons $\sigma(t)=\{\lambda(t)\}$ consisting of poles is studied: [39], 2], 8] deal with semisimple eigenvalues $\lambda(t)$ - which means that the order $m(t)$ of the pole in (1.2) is equal to 1 for every $t \in I$ - and [25], under analyticity conditions, deals with not necessarily semisimple eigenvalues $\lambda(t)$ of finite algebraic multiplicity $m_{0}$. In the present paper the spectral subsets $\sigma(t)$ are also allowed to consist of essential singularities $\lambda(t)$ of the resolvent of $A(t)$ (Example 3.4).

In the adiabatic theorems without spectral gap condition (Section 4 and 5.2), it will be assumed that $\sigma(t)=\{\lambda(t)\}$ for eigenvalues $\lambda(t) \in \partial \sigma(A(t))$, and the relation between $P(t)$ and $A(t), \sigma(t)$ - vaguely described in Condition 1.3 above - will, fairly naturally, be assumed to be as follows: for almost every $t \in I, P(t)$ is a bounded projection commuting with $A(t)$, such that $P(t) D(A(t))=P(t) X$ and

$$
\begin{gathered}
\left.A(t)\right|_{P(t) D(A(t))}-\lambda(t) \text { is nilpotent whereas }\left.A(t)\right|_{(1-P(t)) D(A(t))}-\lambda(t) \text { is injective } \\
\text { with dense range in }(1-P(t)) X .
\end{gathered}
$$

(We will, more briefly, call such projections weakly associated with $A(t)$ and $\lambda(t)$.) Such projections do not always exist in the case without spectral gap - but in case of existence they are uniquely determined by $A(t)$ and $\lambda(t)$. Indeed (by the first theorem of Section 2.1), such projections satisfy

$$
P(t) X=\operatorname{ker}(A(t)-\lambda(t))^{k} \quad \text { and } \quad(1-P(t)) X=\overline{\operatorname{rg}(A(t)-\lambda(t))^{k}}
$$

for all $k \in \mathbb{N}$ with $k \geq m(t)$, where $m(t)$ is the order of nilpotence. Apart from regularity conditions and some resolvent estimate, the assumption that $P(t)$ be weakly associated with $A(t)$ and $\lambda(t) \in \partial \sigma(A(t))$ is already everything that has to be required in the adiabatic theorems without spectral gap condition of the present paper. In [8] and [46]which, so far, are the only works establishing adiabatic theorems without spectral gap for not necessarily skew self-adjoint operators $A(t)$ - the more special situation is studied where the $\lambda(t)$ are weakly semisimple eigenvalues in the sense that the order $m(t)$ of 
nilpotence in (1.3) is equal to 1 for almost every $t \in I$. In numerous other papers, adiabatic theorems without spectral gap for skew self-adjoint operators $A(t)$ have been established - both for eigenvalues $\lambda(t)$ (as in [7], [13], [52, [53], [1]) and for resonances $\lambda(t)$ (as in [3], [19]).

As has already been indicated, we will develop here - in contrast to the existing literature - adiabatic theorems for general operators $A(t)$ both in the case of time-independent domains (Section 3 and 4) and in the case of time-dependent domains (Section 5), which two kinds of adiabatic theorems are related as follows: if in the presented adiabatic theorems for time-independent domains the regularity conditions are strenghtened, then these theorems become special cases of the respective adiabatic theorems for time-dependent domains. We organize the theory in such a way that - apart from some few modifications - the proofs carry over from the case of time-independent to the case of time-dependent domains. Section 5.3 is devoted to an extension of the adiabatic theorem of higher order (also called superadiabatic theorem) from [24] or [40] to the case of time-dependent domains, which extension - albeit a bit technical - is not difficult given the preliminaries of Section 2.

And finally, in Section 6, we apply the general adiabatic theorems for time-dependent domains of Section 5 to obtain - in a very simple way - adiabatic theorems with and without spectral gap condition (Section 6.2 and 6.3) for the special case where the $A(t)=$ $i A_{a(t)}$ are skew self-adjoint operators defined by symmetric sesquilinear forms $a(t)$ having a time-independent form domain. In particular, the adiabatic theorem from [13 is a special case of the theorem in Section 6.3, but we obtain it in a completely different and also simpler way.

All presented adiabatic theorems are furnished with fairly mild regularity conditions on $t \mapsto A(t), \sigma(t), P(t)$. Indeed, in the adiabatic theorems for time-independent domains, for instance, it suffices to require of $A$ that $t \mapsto A(t)$ be $W_{*}^{1, \infty}$-regular (Section 2.1) and $(M, 0)$-stable (Section 2.2), which two things are satisfied if, for instance, $t \mapsto A(t) x$ is continuously differentiable for all $x \in D=D(A(t))$ and $A(t)$ for every $t \in I$ generates a contraction semigroup in $X$. In particular, these regularity conditions are more general than those of the previously known adiabatic theorems. See the discussion after Theorem 2.11 and also see the examples (especially Example 3.3).

We complement the presented adiabatic theorems with examples in order to explore their scope and to demonstrate that some selected hypotheses are essential. All these examples will be of a simple standard structure explained in Section 2.4.

A few words on the interdependence of the various sections of this paper seem appropriate: the sections about the case with spectral gap (Section 3.1, 3.2, 5.1, 6.2 (interdependent in that order)) are independent of the sections about the case without spectral gap (Section 4.1, 4.2, 5.2, 6.3 (interdependent in that order)), and Section 5.3 can be read independently of all sections other than Section 2. Section 2 provides the most important preliminaries, most importantly, those on spectral theory (Section 2.1), well-posedness and evolution systems (Section 2.2), and adiabatic evolution systems (Section 2.3), but also those related to the regularity conditions of our adiabatic theorems. At 
first reading, however, one may well ignore the less common notions of $W_{*}^{m, \infty}$-regularity or $(M, 0)$-stability (explained in Section 2.1 and 2.2) and replace them by the simpler notions of $m$ times strong continuous differentiability and contraction semigroups.

In the entire paper, $X, Y, Z$ denote Banach spaces over $\mathbb{C}$ and NOT, SOT, WOT stand for the norm, strong, weak operator topology of the Banach space $L(X, Y)$ of bounded linear operators from $X$ to $Y$ or - unless explicitly stated otherwise - of the Banach space $L(X)=L(X, X)$. We will abbreviate

$$
I:=[0,1] \quad \text { and } \quad \Delta:=\left\{(s, t) \in I^{2}: s \leq t\right\},
$$

and for evolution systems $U$ defined on $\Delta$ we will write $U(t):=U(t, 0)$ for all $t \in I$, while $U_{\varepsilon}$ for $\varepsilon \in(0, \infty)$ will always denote the evolution system for $\frac{1}{\varepsilon} A$ on $D(A(t))$ provided it exists. As far as notational conventions on general spectral theory are concerned (in particular, concerning the not completely universal notion of continuous and residual spectrum), we follow the standard textbooks [18], [51] or [54]. And finally, the notation employed in the examples will be explained in Section 2.4 .

\section{Some preliminaries}

In this section, we provide the most important preliminaries for the adiabatic theorems of the subsequent sections. Section 2.1 introduces the central spectral-theoretical notions of associatedness and weak associatedness including the notion of weak semisimplicity, properly defines uniform and non-uniform spectral gaps, explains the notion of $W_{*}^{m, \infty}$ regularity of operator-valued maps (including its well-behavedness under the formation of products and inverses), and finally furnishes continually used lemmas centering on one-sided differentiability. Section 2.2 then recalls the concept of well-posedness and evolution systems for non-autonomous linear evolution equations as well as the notion of $(M, 0)$-stability due to Kato. And finally, Section 2.3 introduces the terminology of adiabatic evolution systems while Section 2.4 explains the standard structure of the examples of this paper.

\subsection{Spectral theory, uniform and non-uniform spectral gaps, regularity properties of operator-valued functions, and one-sided differentiability}

As is clear from the setting described in Section 1, a first important point in general adiabatic theory is to precisely define what it means for projections $P(t)$ to be spectrally related to $A(t)$ and $\sigma(t)$ in the sense of Condition 1.3. In order to do so we introduce the following notion of associatedness (which is completely canonical) and weak associatedness (which - for non-normal, or at least, non-spectral operators is not canonical). Suppose $A: D(A) \subset X \rightarrow X$ is a densely defined closed linear map with $\rho(A) \neq \emptyset, \sigma \neq \emptyset$ is a compact isolated subset of $\sigma(A), \lambda$ a not necessarily isolated spectral value of $A$, and $P$ a bounded projection in $X$. We then say, following [51, that $P$ is associated with $A$ and $\sigma$ if and only if $P$ commutes with $A$ (in short: $P A \subset A P$ ), $P D(A)=P X$ and

$$
\sigma\left(\left.A\right|_{P D(A)}\right)=\sigma \quad \text { whereas } \quad \sigma\left(\left.A\right|_{(1-P) D(A)}\right)=\sigma(A) \backslash \sigma .
$$


We say that $P$ is weakly associated with $A$ and $\lambda$ if and only if $P$ commutes with $A$, $P D(A)=P X$ and

$$
\begin{gathered}
\left.A\right|_{P D(A)}-\lambda \text { is nilpotent whereas }\left.A\right|_{(1-P) D(A)}-\lambda \text { is injective and } \\
\text { has dense range in }(1-P) X .
\end{gathered}
$$

If above the order of nilpotence is at most $m$, we will often, more precisely, speak of $P$ as being weakly $m$-associated with $A$ and $\lambda$. Also, we call $\lambda$ a weakly semisimple eigenvalue of $A$ if and only if $\lambda$ is an eigenvalue and there is a projection $P$ weakly 1 -associated with $A$ and $\lambda$.

We point out that, for spectral values $\lambda$ of a densely defined operator $A$ with $\rho(A) \neq \emptyset$ and bounded projections $P$ commuting with $A$, it is fairly natural to take the vaguely described spectral relatedness of Condition 1.3 to mean precisely that $P$ is weakly associated with $A$ and $\lambda$. Indeed, it is more than natural to take this condition to mean at least that $\left.A\right|_{P D(A)}$ is bounded with $\sigma\left(\left.A\right|_{P D(A)}\right)=\{\lambda\}$ (or in other words, that $P D(A)=P X$ and $\left.A\right|_{P D(A)}-\lambda$ is quasinilpotent) and that $\lambda \notin \sigma_{p}\left(\left.A\right|_{(1-P) D(A)}\right)$. (It is important to notice here that, if $\lambda$ is non-isolated in $\sigma(A)$, then it must belong to $\sigma\left(\left.A\right|_{(1-P) D(A)}\right)$ by the closedness of spectra.) And then it is natural to furhter require that $\left.A\right|_{P D(A)}-\lambda$ be even nilpotent (instead of only quasinilpotent) and that $\lambda$ belong to the continuous (instead of the residual) spectrum of $\left.A\right|_{(1-P) D(A)}$, which finally is nothing but the weak associatedness of $P$ with $A$ and $\lambda$.

It is well-known that if $A$ is densely defined with $\rho(A) \neq \emptyset$ and $\sigma \neq \emptyset$ is compact and isolated in $\sigma(A)$, then there exists a unique projection $P$ associated with $A$ and $\sigma$, namely

$$
P:=\frac{1}{2 \pi i} \int_{\gamma}(z-A)^{-1} d z
$$

(occasionally called the Riesz projection of $A$ onto $\sigma$ ), where $\gamma$ is a cycle in $\rho(A)$ with indices $\mathrm{n}(\gamma, \sigma)=1$ and $\mathrm{n}(\gamma, \sigma(A) \backslash \sigma)=0$. (See, for instance, Theorem 2.14 and Proposition 2.15 of [46] or the standard textbook [23] for detailed proofs of these well-known facts.) Also, if $\sigma=\{\lambda\}$ consists of a pole of $(.-A)^{-1}$ of order $m$ and if $P$ is associated with $A$ and $\sigma$, then

$$
P X=\operatorname{ker}(A-\lambda)^{k} \quad \text { and } \quad(1-P) X=\operatorname{rg}(A-\lambda)^{k}
$$

for all $k \in \mathbb{N}$ with $k \geq m$ (Theorem 5.8-A of [50]). We now prove a similar theorem for weak associatedness revealing, in particular, that on the one hand, for a given operator $A$ and a spectral value $\lambda$ of $A$ no weakly associated projection need exist in general and that, on the other hand, if there exists any weakly associated projection then it is already unique. (See the third remark following the theorem.) This theorem complements Corollary 2.2 of [36], which covers only the case of isolated spectral values. It will be crucial in the presented adiabatic theorems without spectral gap condition. 
Theorem 2.1. Suppose $A: D(A) \subset X \rightarrow X$ is a densely defined closed linear map with $\rho(A) \neq \emptyset, \lambda \in \sigma(A)$, and $P$ is a bounded projection in $X$. If $P$ is weakly $m$-associated with $A$ and $\lambda$, then

$$
P X=\operatorname{ker}(A-\lambda)^{k} \quad \text { and } \quad(1-P) X=\overline{\operatorname{rg}(A-\lambda)^{k}}
$$

for all $k \in \mathbb{N}$ with $k \geq m$ and, moreover, $\lambda$ is a pole of $(.-A)^{-1}$ if and only if $\operatorname{rg}(A-\lambda)^{k}$ is closed for some (all) $k \geq m$, in which case $P$ is the projection associated with $A$ and $\lambda$.

Proof. We may clearly assume that $\lambda=0$ because $P$, being weakly associated with $A$ and $\lambda$, is also weakly associated with $A-\lambda$ and 0 . Set $M:=P X$ and $N:=(1-P) X$. We first show that $M=\operatorname{ker} A^{k}$ for all $k \geq m$. Since $\left.A\right|_{P X}=\left.A\right|_{P D}$ is nilpotent of order $m,\left.A^{k}\right|_{P X}=\left(\left.A\right|_{P X}\right)^{k}=0$ and hence $M=P X \subset \operatorname{ker} A^{k}$ for all $k \geq m$. And since $\left.A\right|_{(1-P) D(A)}$ is injective,

$$
\left.A^{k}\right|_{(1-P) D\left(A^{k}\right)}=\left(\left.A\right|_{(1-P) D(A)}\right)^{k}
$$

is injective as well and hence $\operatorname{ker} A^{k} \subset P X=M$ for all $k \in \mathbb{N}$. We now show that $N=\overline{\operatorname{rg} A^{k}}$ for all $k \geq m$. As $P X=\operatorname{ker} A^{k}$ for $k \geq m$, we have

$$
\operatorname{rg} A^{k}=A^{k} P D\left(A^{k}\right)+A^{k}(1-P) D\left(A^{k}\right)=(1-P) A^{k} D\left(A^{k}\right) \subset(1-P) X=N
$$

and therefore $\overline{\operatorname{rg} A^{k}} \subset N$ for all $k \geq m$. It remains to show that the reverse inclusion $N \subset \overline{\operatorname{rg} A^{k}}$ holds true for all $k \in \mathbb{N}$ and this will be done by induction over $k$. Since $\left.A\right|_{(1-P) D(A)}$ has dense range in $(1-P) X=N$, the desired inclusion is clearly satisfied for $k=1$. Suppose now that $N \subset \overline{\operatorname{rg} A^{k}}$ is satisfied for some arbitrary $k \in \mathbb{N}$. Since

$$
\left.\operatorname{rg} A\right|_{(1-P) D(A)}=A(1-P) D(A)=A\left(z_{0}-A\right)^{-1} N
$$

and since $A\left(z_{0}-A\right)^{-1}$ is a bounded operator for every $z_{0} \in \rho(A)$, it then follows by the induction hypothesis that $A\left(z_{0}-A\right)^{-1} N \subset \overline{\operatorname{rg} A^{k+1}}$ and hence

$$
N=\overline{\left.\operatorname{rg} A\right|_{(1-P) D(A)}} \subset \overline{\operatorname{rg} A^{k+1}},
$$

as desired. In order to see the characterization of the pole property for $\lambda=0$ (under the weak associatedness hypothesis), we have only to observe that, by what has just been shown, the ascent of $A$ is less than or equal to $m$ and to use Theorem 5.8-A and Theorem 5.8-D of [50] or Theorem V.10.1 and Theorem V.10.2 of [51].

Some remarks, answering in particular the existence and uniqueness question for weak associatedness, are in order.

1. We also have the following simple converse of the above theorem: if one has a direct sum decomposition

$$
X=\operatorname{ker}(A-\lambda)^{m} \oplus \overline{\operatorname{rg}(A-\lambda)^{m}}
$$


for some $m \in \mathbb{N}$ (where $A: D(A) \subset X \rightarrow X$ is densly defined with $\rho(A) \neq \emptyset$ and $\lambda \in \sigma(A))$ and and if the projection $P$ corresponding to the above decomposition of $X$ commutes with $A$, then $P$ is weakly $m$-associated with $A$ and $\lambda$. (It should be noticed here that $P$ is automatically bounded because $(A-\lambda)^{m}$ is closed by $\rho(A) \neq \emptyset$.)

2. It is easy to see - with the help of Volterra operators and shift operators (Section 2.4) as building blocks - that the nilpotence, injectivity, and dense range requirements (encapsulated in the weak associatedness) are all essential for the conclusion of the above theorem. And - as opposed to the case where $A$ is normal - isolatedness of $\lambda$ in $\sigma(A)$ alone does not imply the closedness of the spaces $\operatorname{rg}(A-\lambda)^{k}$ in the theorem above. (Just take $A:=\operatorname{diag}(0, V)$ on $X:=L^{2}(I) \times L^{2}(I)$ with the Volterra operator $V$ defined by $(V f)(t):=\int_{0}^{t} f(\tau) d \tau, \lambda:=0$, and $P$ the orthogonal projection onto $L^{2}(I) \times 0$.)

3. If $A: D(A) \subset X \rightarrow X$ is densely defined with $\rho(A) \neq \emptyset$ and $\lambda \in \sigma(A)$, then the above theorem implies the following two facts about weak associatedness. (i) In general, there exists no projection weakly associated with $A$ and $\lambda$. (Indeed, for $A:=\operatorname{diag}(0, S)$ on $X:=\ell^{2}(\mathbb{N}) \times \ell^{2}(\mathbb{N})$ and $\lambda:=0$, where $S$ is the right shift operator on $\ell^{2}(\mathbb{N})$, one has

$$
\operatorname{ker}(A-\lambda)^{k}+\overline{\operatorname{rg}(A-\lambda)^{k}} \neq X
$$

for all $k \in \mathbb{N}$. Also see the remarks after Example 4.7 and Example 5.8) (ii) If, however, there exists a projection weakly associated with $A$ and $\lambda$, then there is only one such projection. (Indeed, if $P$ and $Q$ are two projections weakly associated with $A$ and $\lambda$ and if the orders of nilpotence of $\left.A\right|_{P D(A)}-\lambda$ and $\left.A\right|_{Q D(A)}-\lambda$ are $m$ and $n$ respectively, then

$$
\begin{gathered}
P X=\operatorname{ker}(A-\lambda)^{m}=\operatorname{ker}(A-\lambda)^{n}=Q X, \\
(1-P) X=\overline{\operatorname{rg}(A-\lambda)^{m}}=\overline{\operatorname{rg}(A-\lambda)^{n}}=(1-Q) X
\end{gathered}
$$

and therefore $P=Q$.)

A rather well-understood general class of operators $A$ for which the existence of weakly associated projections is always guaranteed is furnished by the class of spectral operators of scalar type (Definition XVIII.2.12 in [18]) or bounded spectral operators of finite type (Definition XV.5.3 in [18]). (Spectral operators of scalar type comprise, for instance, the normal operators or the generic one-dimensional periodic Schrödinger operators (Remark 8.7 of [22]).) In both cases the weakly associated projections are just given by $P^{A}(\{\lambda\})$ where $P^{A}$ denotes the spectral measure - or, in the terminology of [18], the spectral resolution - of the spectral operator $A$ (Definition XV.2.5 and XVIII.2.1 of [18]). (In order to see this, use Theorem XVIII.2.11 of [18] in the former case and in the latter case notice that

$$
\begin{gathered}
\left.A\right|_{P X}-\lambda \text { is quasinilpotent whereas }\left.A\right|_{(1-P) X}-\lambda \text { is injective and } \\
\text { has dense range in }(1-P) X
\end{gathered}
$$

by Corollary XV.8.5 of [18] and that the quasinilpotence above is even a nilpotence by the finite type assumption.) We point out, however, that adiabatic theory is by no means confined to spectral operators $A(t)$ (Example 4.7). 
Associatedness and weak associatedness, for isolated spectral values $\lambda$ of an operator $A$, are related as follows. If $\lambda$ is a pole of $(.-A)^{-1}$, then associatedness and weak associatedness coincide: a projection $P$ is then associated with $A$ and $\lambda$ if and only if it is weakly associated with $A$ and $\lambda$ (by Theorem 2.1 and the first remark following it). If, however, $\lambda$ is an essential singularity of $(.-A)^{-1}$, then associatedness and weak associatedness have nothing to do with each other: a projection $P$ associated with $A$ and $\lambda$ can then not possibly be weakly associated with $A$ and $\lambda$, and vice versa. (Indeed, if a projection $P$ is both associated and weakly $m$-associated with $A$ and $\lambda$, then

$$
\begin{aligned}
z \mapsto(z-A)^{-1} & =(z-A)^{-1} P+(z-A)^{-1}(1-P) \\
& =\sum_{k=0}^{m-1} \frac{\left(\left.A\right|_{P D(A)}-\lambda\right)^{k}}{(z-\lambda)^{k+1}} P+\left(z-\left.A\right|_{(1-P) D(A)}\right)^{-1}(1-P)
\end{aligned}
$$

has a pole of order $m$ at $\lambda$.) Additionally, since semisimple spectral values of $A$ are, by definition, poles of $(.-A)^{-1}$ of order 1 (and, in particular, eigenvalues), it follows that semisimplicity and weak semisimplicity coincide for isolated points $\lambda$ of $\sigma(A)$ that are poles.

We close this paragraph on spectral theory by noting that in reflexive spaces weak associatedness carries over to the dual maps - provided that some core condition is satisfied, which is the case for semigroup generators, for instance (Proposition II.1.8 of [20]). In the presented adiabatic theorem without spectral gap condition for reflexive spaces, this will be important. Associatedness carries over to dual maps as well, of course (Section III.6.6 of [31]) - but this will not be needed in the sequel.

Proposition 2.2. Suppose $A: D(A) \subset X \rightarrow X$ is a densely defined closed linear map in the reflexive space $X$ such that $\rho(A) \neq \emptyset$ and $D\left(A^{k}\right)$ is a core for $A$ for all $k \in \mathbb{N}$. If $P$ is weakly m-associated with $A$ and $\lambda \in \sigma(A)$, then $P^{*}$ is weakly m-associated with $A^{*}$ and $\lambda$.

Proof. We begin by showing - by induction over $k \in \mathbb{N}$ - the preparatory statement that

$$
\left(A^{k}\right)^{*}=\left(A^{*}\right)^{k}
$$

for all $k \in \mathbb{N}$, which might also be of independent interest (notice that $D\left(A^{k}\right)$ being a core for $A$ is dense in $X$, so that $\left(A^{k}\right)^{*}$ is really well-defined). Clearly, (2.1) is true for $k=1$ and, assuming that it is true for some arbitrary $k \in \mathbb{N}$, we now show that $\left(A^{k+1}\right)^{*}=\left(A^{*}\right)^{k+1}$ holds true as well. It is easy to see that $\left(A^{*}\right)^{k+1} \subset\left(A^{k+1}\right)^{*}$ and it remains to see that $D\left(\left(A^{k+1}\right)^{*}\right) \subset D\left(\left(A^{*}\right)^{k+1}\right)$. So let $x^{*} \in D\left(\left(A^{k+1}\right)^{*}\right)$. We show that

$$
x^{*} \in D\left(\left(A^{k}\right)^{*}\right) \text { and }\left(A^{k}\right)^{*} x^{*} \in D\left(A^{*}\right),
$$

from which it then follows - by the induction hypothesis - that $x^{*} \in D\left(\left(A^{*}\right)^{k+1}\right)$ as desired. In order to prove that $x^{*} \in D\left(\left(A^{k}\right)^{*}\right)$ we show that

$$
x^{*} \in D\left(\left(A^{l}\right)^{*}\right)
$$


for all $l \in\{1, \ldots, k\}$ - by induction over $l \in\{1, \ldots, k\}$ and by working with suitable powers of $\left(A^{*}-z_{0}\right)^{-1}=\left(\left(A-z_{0}\right)^{-1}\right)^{*}$, where $z_{0}$ is an arbitrary point of $\rho\left(A^{*}\right)=\rho(A) \neq \emptyset$ (Theorem III.5.30 of [31]). In the base step of the induction, notice that for all $y \in D(A)$

$$
\begin{aligned}
& \left\langle\left(A^{*}-z_{0}\right)^{-k}\left(A^{k+1}\right)^{*} x^{*}, y\right\rangle=\left\langle x^{*}, A^{k+1}\left(A-z_{0}\right)^{-k} y\right\rangle \\
& =\left\langle x^{*},\left(A-z_{0}\right) y\right\rangle+\sum_{i=0}^{k}\left(\begin{array}{c}
k+1 \\
i
\end{array}\right) z_{0}^{k+1-i}\left\langle\left(A^{*}-z_{0}\right)^{-k+i} x^{*}, y\right\rangle,
\end{aligned}
$$

from which it follows that $x^{*} \in D\left(\left(A-z_{0}\right)^{*}\right)=D\left(A^{*}\right)$. In the inductive step, assume that $x^{*} \in D\left(A^{*}\right), \ldots, D\left(\left(A^{l}\right)^{*}\right)$ for some arbitrary $l \in\{1, \ldots, k-1\}$. Since for all $y \in D\left(A^{l+1}\right)$

$$
\begin{aligned}
\left\langle\left(A^{*}-z_{0}\right)^{-(k-l)}\left(A^{k+1}\right)^{*} x^{*}, y\right\rangle=\left\langle x^{*}, A^{k+1}\left(A-z_{0}\right)^{-(k-l)} y\right\rangle & \\
= & \left\langle x^{*},\left(A-z_{0}\right)^{l+1} y\right\rangle+\sum_{i=k-l+1}^{k}\left(\begin{array}{c}
k+1 \\
i
\end{array}\right) z_{0}^{k+1-i}\left\langle x^{*},\left(A-z_{0}\right)^{-(k-l)+i} y\right\rangle \\
& +\sum_{i=0}^{k-l}\left(\begin{array}{c}
k+1 \\
i
\end{array}\right) z_{0}^{k+1-i}\left\langle\left(A^{*}-z_{0}\right)^{-(k-l)+i} x^{*}, y\right\rangle,
\end{aligned}
$$

it follows by the induction hypothesis of the $l$-induction and by applying the binomial formula to $\left(A-z_{0}\right)^{-(k-l)+i} y$ for $i \in\{k-l+1, \ldots, k+1\}$ that $x^{*} \in D\left(\left(A^{l+1}\right)^{*}\right)$. So the $l$-induction is finished and it remains to show that $\left(A^{k}\right)^{*} x^{*} \in D\left(A^{*}\right)$. Since $D\left(A^{k+1}\right)$ by assumption is a core for $A$, there is for every $y \in D(A)$ a sequence $\left(y_{n}\right)$ in $D\left(A^{k+1}\right)$ such that

$$
\left\langle\left(A^{k}\right)^{*} x^{*}, A y\right\rangle=\lim _{n \rightarrow \infty}\left\langle\left(A^{k}\right)^{*} x^{*}, A y_{n}\right\rangle=\lim _{n \rightarrow \infty}\left\langle x^{*}, A^{k+1} y_{n}\right\rangle=\left\langle\left(A^{k+1}\right)^{*} x^{*}, y\right\rangle .
$$

It follows that $\left(A^{k}\right)^{*} x^{*} \in D\left(A^{*}\right)$ and this yields - together with the induction hypothesis of the $k$-induction - that $x^{*} \in D\left(\left(A^{*}\right)^{k+1}\right)$, which finally ends the proof the preparatory statement (2.1).

After this preparation we can now move on to the main part of the proof where we assume, without loss of generality, that $\lambda=0$ and exploit the first remark after Theorem 2.1 to show that $P^{*}$ is weakly $m$-associated with $A^{*}$ and $\lambda=0 . A^{*}$ is densely defined (due to the reflexivity of $X$ (Theorem III.5.29 of [31])) with $\rho\left(A^{*}\right)=\rho(A) \neq \emptyset$ (Theorem III.5.30 of [31]) and

$$
P^{*} A^{*} \subset(A P)^{*} \subset(P A)^{*}=A^{*} P^{*}
$$

because $A P \supset P A$. Since $\left(A^{m}\right)^{*}=\left(A^{*}\right)^{m}$ by (2.1) and since $P X=\operatorname{ker} A^{m}$ and $(1-$ $P) X=\overline{\operatorname{rg} A^{m}}$ (by Theorem 2.1), we further have

$$
\begin{gathered}
P^{*} X^{*}=\operatorname{ker}(1-P)^{*}=((1-P) X)^{\perp}=\left(\overline{\operatorname{rg} A^{m}}\right)^{\perp}=\operatorname{ker}\left(A^{m}\right)^{*}=\operatorname{ker}\left(A^{*}\right)^{m} \\
\text { and } \\
\left(1-P^{*}\right) X^{*}=\operatorname{ker} P^{*}=(P X)^{\perp}=\left(\operatorname{ker} A^{m}\right)^{\perp}=\left(\operatorname{ker}\left(A^{m}\right)^{* *}\right)_{\perp}=\overline{\operatorname{rg}\left(A^{m}\right)^{*}}=\overline{\operatorname{rg}\left(A^{*}\right)^{m}}
\end{gathered}
$$


where in the fourth equality of the second line the closedness of $A^{m}$ (following from $\rho(A) \neq \emptyset$ ) and the reflexivity of $X$ have been used. (In the above relations, we denote by $U^{\perp}:=\left\{x^{*} \in Z^{*}:\left\langle x^{*}, U^{*}\right\rangle=0\right\}$ and $V_{\perp}:=\{x \in Z:\langle V, x\rangle=0\}$ the annihilators of subsets $U$ and $V$ of a normed space $Z$ and its dual $Z^{*}$, respectively.) It is now clear from the first remark after Theorem 2.1 that $P^{*}$ is weakly $m$-associated with $A^{*}$ and $\lambda=0$ and we are done.

We continue by properly defining what exactly we mean by uniform and non-uniform spectral gaps. Suppose that $A(t): D(A(t)) \subset X \rightarrow X$, for every $t$ in some compact interval $J$, is a densely defined closed linear map and that $\sigma(t)$ is a compact subset of $\sigma(A(t))$ for every $t \in J$. We then speak of a uniform resp. non-uniform spectral gap for $A$ and $\sigma$ if and only if $\sigma(t)$ is isolated in $\sigma(A(t)$ ) for every $t \in J$ (in short: there is a spectral gap for $A$ and $\sigma$ ) and $\sigma($.$) is resp. is not uniformly isolated in \sigma(A($.$) ). (Uniform$ isolatedness means that there is a $t$-independent constant $r_{0}>0$ such that

$$
\bar{U}_{r_{0}}(\sigma(t)) \cap \sigma(A(t)):=\left\{z \in \mathbb{C}: \operatorname{dist}(z, \sigma(t)) \leq r_{0}\right\} \cap \sigma(A(t))=\sigma(t)
$$

for every $t \in I$, of course.) Also, we say that $\sigma($.$) falls into \sigma(A().) \backslash \sigma($.$) at the point$ $t_{0} \in J$ if and only if there is a sequence $\left(t_{n}\right)$ in $J$ converging to $t_{0}$ such that

$$
\operatorname{dist}\left(\sigma\left(t_{n}\right), \sigma\left(A\left(t_{n}\right)\right) \backslash \sigma\left(t_{n}\right)\right) \longrightarrow 0 \quad(n \rightarrow \infty)
$$

It is clear that the set of points at which $\sigma($.$) falls into \sigma(A().) \backslash \sigma($.$) is closed. Also, it$ follows by the compactness of $J$ that a spectral gap for $A$ and $\sigma$ is uniform if and only if $\sigma($.$) at no point falls into \sigma(A().) \backslash \sigma($.$) . The following proposition gives a criterion (in$ terms of some mild regularity conditions on $t \mapsto A(t), \sigma(t), P(t))$ for a spectral gap for $A$ and $\sigma$ to be even uniform. It is of some interest in the third remark at the beginning of Section 3.3 (and is crucial in the applied example to neutron transport theory presented in [46]). We refer to Section IV.2.4 and Theorem IV.2.25 of 31 for a definition and a characterization of convergence (and hence, continuity) in the generalized sense and to Section IV.3 of [31] for the definition of upper and lower semicontinuity of set-valued functions $t \mapsto \sigma(t)$.

Proposition 2.3. Suppose that $A(t): D(A(t)) \subset X \rightarrow X$ is a closed linear map for every $t$ in a compact interval $J$ and that $t \mapsto A(t)$ is continuous in the generalized sense. Suppose further that $\sigma(t)$ for every $t \in J$ is a compact and isolated subset of $\sigma(A(t))$ such that $\sigma($.$) falls into \sigma(A().) \backslash \sigma($.$) at t_{0} \in J$, and let $t \mapsto \sigma(t)$ be upper semicontinuous at $t_{0}$. Finally, for every $t \in J$ let $P(t)$ be the projection associated with $A(t)$ and $\sigma(t)$. Then $t \mapsto P(t)$ is discontinuous at $t_{0}$ and

$$
\limsup _{n \rightarrow \infty}\left(\operatorname{rk} P\left(t_{n}\right)\right) \leq \operatorname{rk} P\left(t_{0}\right)-1
$$

for all sequences $\left(t_{n}\right)$ such that $t_{n} \longrightarrow t_{0}$ and $\operatorname{dist}\left(\sigma\left(t_{n}\right), \sigma\left(A\left(t_{n}\right)\right) \backslash \sigma\left(t_{n}\right)\right) \longrightarrow 0$. 
See [46] (Proposition 5.3) for a proof. Clearly, one also has the following converse of the above proposition: if $t \mapsto A(t)$ is continuous in the generalized sense as above and $t \mapsto \sigma(t)$ is even continuous (that is, upper and lower semicontinuous) then uniform isolatedness of $\sigma($.$) in \sigma(A()$.$) implies that t \mapsto P(t)$ is continuous. (Use Theorem IV.3.15 of [31].)

We now turn to regularity properties of vector-valued and, in particular, operatorvalued functions. We start by briefly recalling those facts on vector-valued Sobolev spaces that will be needed in the sequel (see [6] and [5] for more detailed expositions). We follow the notational conventions of [6]. In particular, $(\mu$-)measurability of a $Y$-valued map on a complete measure space $\left(X_{0}, \mathcal{A}, \mu\right)$ will not only mean that this map is $\mathcal{A}$-measurable but also that it is $\mu$-almost separably-valued, whereas the notion of $(\mu$-)strong measurability will be reserved for operator-valued maps that are pointwise $\mu$-measurable. Suppose $J$ is a non-trivial interval and $p \in[1, \infty) \cup\{\infty\}$. Then $W^{1, p}(J, X)$ is defined to consist of those (equivalence classes of) $p$-integrable functions $f: J \rightarrow X$ for which there is a $p$-integrable function $g: J \rightarrow X$ (called a weak derivative of $f$ ) such that

$$
\int_{J} f(t) \varphi^{\prime}(t) d t=-\int_{J} g(t) \varphi(t) d t
$$

for all $\varphi \in C_{c}^{\infty}\left(J^{\circ}, \mathbb{C}\right)$. As usual, $p$-integrability of a function $f: J \rightarrow X$ (with $p \in[1, \infty) \cup$ $\{\infty\})$ means that $f$ is measurable and $\|f\|_{p}:=\left(\int_{J}\|f(\tau)\|^{p} d \tau\right)^{1 / p}<\infty$ if $p \in[1, \infty)$ or $\|f\|_{p}:=\operatorname{ess}^{-\sup _{t \in J}}\|f(t)\|<\infty$ if $p=\infty$. If $f$ is in $W^{1, p}(J, X)$ and $g_{1}, g_{2}$ are two weak derivatives of $f$, then $g_{1}=g_{2}$ almost everywhere, so that up to almost everywhere equality there is only one weak derivative of $f$ which is denoted by $\partial f$. It is well-known that $W^{1, p}(J, X)$ is a Banach space w.r.t. the norm $\|\cdot\|_{1, p}$ whith $\|f\|_{1, p}:=\|f\|_{p}+\|\partial f\|_{p}$ for $f \in W^{1, p}(J, X)$. It is also well-known that the space $W^{1, \infty}(J, X)-$ just like $W^{1, p}(J, X)$ for $p \in[1, \infty)$ - can be characterized by means of indefinite integrals: $W^{1, \infty}(J, X)$ consists of those (equivalence classes of) $\infty$-integrable functions $f: J \rightarrow X$ for which there is an $\infty$-integrable function $g$ such that for some (and hence every) $t_{0} \in J$

$$
f(t)=f\left(t_{0}\right)+\int_{t_{0}}^{t} g(\tau) d \tau \text { for all } t \in J,
$$

or, equivalently (by Lebesgue's differentiation theorem), $W^{1, \infty}(J, X)$ consists of (equivalence classes of) $\infty$-integrable functions $f: J \rightarrow X$ which are differentiable almost everywhere and whose (pointwise) derivative $f^{\prime}$ is $\infty$-integrable such that for some (and hence every) $t_{0} \in J$

$$
f(t)=f\left(t_{0}\right)+\int_{t_{0}}^{t} f^{\prime}(\tau) d \tau \text { for all } t \in J
$$

Additionally, the pointwise derivative $f^{\prime}$ of an $f \in W^{1, \infty}(J, X)$ equals the weak derivative $\partial f$ almost everywhere. It follows from this characterization that, in case $X$ is reflexive (or more generally, satisfies the Radon-Nikodým property), $W^{1, \infty}(J, X)$ consists exactly 
of the (equivalence classes of) Lipschitz continuous functions (where one inclusion is completely trivial and independent of the Radon-Nikodým property, of course).

We now move on to define - following the introduction of Kato's work [32] $-W_{*}^{m, \infty}$ regularity which shall be used in all our adiabatic theorems with time-independent domains. An operator-valued function $J \ni t \mapsto A(t) \in L(X, Y)$ on a compact interval $J$ is said to belong to $W_{*}^{0, \infty}(J, L(X, Y))=L_{*}^{\infty}(J, L(X, Y))$ if and only if $t \mapsto A(t)$ is strongly measurable and $t \mapsto\|A(t)\|$ is essentially bounded. And $t \mapsto A(t)$ is said to belong to $W_{*}^{1, \infty}(J, L(X, Y))$ if and only if there is $B \in L_{*}^{\infty}(J, L(X, Y))$ (called a $W_{*}^{1, \infty}$-derivative of $A$ ) such that for some (and hence every) $t_{0} \in J$

$$
A(t) x=A\left(t_{0}\right) x+\int_{t_{0}}^{t} B(\tau) x d \tau \text { for all } t \in J \text { and } x \in X .
$$

$W_{*}^{m, \infty}(J, L(X, Y))$ for arbitrary $m \in \mathbb{N}$ is defined recursively, of course.

We point out that the $W_{*}^{m, \infty}$-spaces (unlike the $W^{m, \infty}$-spaces), by definition, consist of functions (of operators) rather than equivalence classes of such functions. It is obvious from the characterization of $W^{1, \infty}(J, Y)$ by way of indefinite integrals that, if $t \mapsto A(t)$ is in $W_{*}^{1, \infty}(J, L(X, Y))$, then $t \mapsto A(t) x$ is (the continuous representative of an element) in $W^{1, \infty}(J, Y)$. In particular, $W_{*}^{1, \infty}$-regularity implies Lipschitz continuity w.r.t. the norm topology, and furthermore, $W_{*}^{1, \infty}$-regularity can be thought of as being not much more than Lipschitz continuity (in view of the above remarks in conjunction with the RadonNikodým property). A simple and important criterion for $W_{*}^{1, \infty}$-regularity is furnished by the following proposition.

Proposition 2.4. Suppose $J \ni t \mapsto A(t) \in L(X, Y)$ is SOT-or WOT-continuously differentiable, where $J$ is a compact interval. Then $t \mapsto A(t)$ is in $W_{*}^{1, \infty}(J, L(X, Y))$.

Proof. It is well-known that a weakly continuous map $J \rightarrow Y$ is almost separably valued, whence $t \mapsto A^{\prime}(t) x$ is measurable (by Pettis' characterization of measurability). With the help of the Hahn-Banach theorem the conclusion readily follows.

It follows from Lebesgue's differentiation theorem that $W_{*}^{1, \infty}$-derivatives are essentially unique, more precisely: if $t \mapsto A(t)$ is in $W_{*}^{1, \infty}(J, L(X, Y))$ and $B_{1}, B_{2}$ are two $W_{*}^{1, \infty}$ derivatives of $A$, then one has for every $x \in X$ that $B_{1}(t) x=B_{2}(t) x$ for almost every $t \in J$. It should be emphasized that this last condition does not imply that $B_{1}(t)=B_{2}(t)$ for almost every $t \in J$. (Indeed, take $J:=[0,1], X:=\ell^{2}(J)$ and define

$$
A(t):=0 \text { as well as } B_{1}(t) x:=\left\langle e_{t}, x\right\rangle e_{t} \text { and } B_{2}(t) x:=0
$$

for $t \in J$ and $x \in X$, where $e_{t}(s):=\delta_{s t}$. Then, for every $x \in X, B_{1}(t) x$ is different from 0 for at most countably many $t \in J$, and it follows that $B_{1}$ and $B_{2}$ both are $W_{*}^{1, \infty}$-derivatives of $A$, but $B_{1}(t) \neq B_{2}(t)$ for every $t \in J$.)

In the presented adiabatic theorems for time-independent domains (Section 3 and 4), we will make much use of the following lemma stating that $W_{*}^{1, \infty}$-regularity carries over to products and inverses. It is noted in the introduction of [32] for separable spaces. 
We prove it here since it is not proved in [32] and, more importantly, since it is not a priori clear - (almost) separability being crucial for measurability - that the separability assumption of [32] is actually not needed for this lemma. An analogue of this lemma for SOT- and WOT-continuous differentiability is well-known (and easily proved with the help of the theorem of Banach-Steinhaus).

Lemma 2.5. (i) Suppose that $t \mapsto A(t)$ is in $W_{*}^{1, \infty}(J, L(X, Y))$ and that $t \mapsto B(t)$ is in $W_{*}^{1, \infty}(J, L(Y, Z))$ where $J=[a, b]$. Then $t \mapsto B(t) A(t)$ is in $W_{*}^{1, \infty}(J, L(X, Z))$ and $t \mapsto B^{\prime}(t) A(t)+B(t) A^{\prime}(t)$ is a $W_{*}^{1, \infty}$-derivative of $B A$ for every $W_{*}^{1, \infty}$-derivative $A^{\prime}, B^{\prime}$ of $A$ or $B$, respectively.

(ii) Suppose that $t \mapsto A(t)$ is in $W_{*}^{1, \infty}(J, L(X, Y))$ and that $A(t)$ is bijective onto $Y$ for every $t \in J$. Then $t \mapsto A(t)^{-1}$ is in $W_{*}^{1, \infty}(J, L(Y, X))$ and $t \mapsto-A(t)^{-1} A^{\prime}(t) A(t)^{-1}$ is a $W_{*}^{1, \infty}$-derivative of $A^{-1}$ for every $W_{*}^{1, \infty}$-derivative $A^{\prime}$ of $A$.

Proof. We begin with some general preparatory considerations. Whenever an operatorvalued map $t \mapsto C(t)$ is in $W_{*}^{1, \infty}(J, L(X, Y))$, we shall write $\tilde{C}$ for the map on $\tilde{J}:=$ $[a-1, b+1]$ obtained from $C$ by trivially extending it by $C(a)$ to the left and by $C(b)$ to the right. Also we shall write

$$
\tilde{C}_{n}(t) x:=\left(j_{\frac{1}{n}} * \tilde{C}(.) x\right)(t)=\int_{\tilde{J}} j_{\frac{1}{n}}(t-r) \tilde{C}(r) x d r
$$

for all $t \in \tilde{J}$ and all $x \in X$. It is clear that $t \mapsto \tilde{C}(t)$ is in $W_{*}^{1, \infty}(\tilde{J}, L(X, Y))$. In particular, $t \mapsto \tilde{C}(t) x$ is the continuous representative of an element of $W^{1, \infty}(\tilde{J}, Y)$ and hence of $W^{1,1}(\tilde{J}, Y)$ for every $x \in X$, from which we conclude - using the usual facts on mollification (which are proved in exactly the same way as in the scalar case) - that for every $x \in X$

$$
\begin{gathered}
\left.\left.\tilde{C}_{n}(.) x\right|_{J} \underset{\|\cdot\|_{\infty}}{\longrightarrow} \tilde{C}(.) x\right|_{J}=C(.) x \quad(n \rightarrow \infty), \\
\left\|\tilde{C}_{n}(t) x\right\| \leq \sup _{\tau \in \tilde{J}}\|\tilde{C}(\tau)\|\|x\| \quad(t \in J, n \in \mathbb{N}), \\
\left\|\tilde{C}_{n}^{\prime}(t) x\right\|=\left\|\left(j_{\frac{1}{n}} * \tilde{C}^{\prime}(.) x\right)(t)\right\| \leq \underset{\tau \in \tilde{J}}{\operatorname{ess-sup}}\left\|\tilde{C}^{\prime}(\tau)\right\|\|x\| \quad(t \in J, n \in \mathbb{N}), \\
\left.\left.\tilde{C}_{n}(.) x\right|_{J} \underset{\|\cdot\|_{1,1}}{\longrightarrow} \tilde{C}(.) x\right|_{J}=C(.) x \quad(n \rightarrow \infty),
\end{gathered}
$$

where $\tilde{C}^{\prime}$ denotes an arbitrary $W_{*}^{1, \infty}$-derivative of $\tilde{C}$.

(i) We fix arbitrary $W_{*}^{1, \infty}$-derivatives $A^{\prime}, B^{\prime}$ of $A$ and $B$ and prove that $t \mapsto B^{\prime}(t) A(t)+$ $B(t) A^{\prime}(t)$ is in $L_{*}^{\infty}(J, L(X, Z))$ and that

$$
B(t) A(t) x=B(a) A(a) x+\int_{a}^{t} B^{\prime}(\tau) A(\tau) x+B(\tau) A^{\prime}(\tau) x d \tau
$$


for every $t \in J$ and $x \in X$. It is easy to see that $t \mapsto B^{\prime}(t) A(t)+B(t) A^{\prime}(t)$ is indeed in $L_{*}^{\infty}(J, L(X, Z)$ ) (by virtue of Lemma A 4 of [30] stating that strong measurability of operator-valued functions carries over to products). Additionally, it is clear from the SOT-continuous differentiability of $t \mapsto \tilde{A}_{n}(t)$ and $t \mapsto \tilde{B}_{n}(t)$ that

$$
\tilde{B}_{n}(t) \tilde{A}_{n}(t) x=\tilde{B}_{n}(a) \tilde{A}_{n}(a) x+\int_{a}^{t} \tilde{B}_{n}^{\prime}(\tau) \tilde{A}_{n}(\tau) x+\tilde{B}_{n}(\tau) \tilde{A}_{n}^{\prime}(\tau) x d \tau
$$

for every $t \in J, x \in X$, and $n \in \mathbb{N}$. We now fix $t \in J$ and $x \in X$ and choose $\tilde{a}, \tilde{b}$ such that $\|A(\tau)\|,\left\|A^{\prime}(\tau)\right\| \leq \tilde{a}$ and $\|B(\tau)\|,\left\|B^{\prime}(\tau)\right\| \leq \tilde{b}$ for almost every $\tau \in J$. In virtue of the preparatory considerations above, we have

$$
\begin{gathered}
\int_{a}^{t} \tilde{B}_{n}(\tau) \tilde{A}_{n}^{\prime}(\tau) x d \tau-\int_{a}^{t} B(\tau) A^{\prime}(\tau) x d \tau \\
=\int_{a}^{t} \tilde{B}_{n}(\tau)\left(\tilde{A}_{n}^{\prime}(\tau) x-A^{\prime}(\tau) x\right) d \tau+\int_{a}^{t}\left(\tilde{B}_{n}(\tau)-B(\tau)\right) A^{\prime}(\tau) x d \tau \longrightarrow 0
\end{gathered}
$$

as $n \rightarrow \infty$. And since for every $\varepsilon>0$ there is a partition $a=t_{0}<t_{1}<\cdots<t_{m}=t$ of $[a, t]$ such that for all $i \in\{1, \ldots, m\}$ one has $\sup _{\tau \in\left[t_{i-1}, t_{i}\right]}\left\|A(\tau) x-A\left(t_{i}\right) x\right\|<\varepsilon / 2 \tilde{b}$ we see that the norm of

$$
\begin{gathered}
\int_{a}^{t} \tilde{B}_{n}^{\prime}(\tau) \tilde{A}_{n}(\tau) x d \tau-\int_{a}^{t} B^{\prime}(\tau) A(\tau) x d \tau \\
=\int_{a}^{t} \tilde{B}_{n}^{\prime}(\tau)\left(\tilde{A}_{n}(\tau) x-A(\tau) x\right) d \tau+\sum_{i=1}^{m} \int_{t_{i-1}}^{t_{i}}\left(\tilde{B}_{n}^{\prime}(\tau)-B^{\prime}(\tau)\right)\left(A(\tau) x-A\left(t_{i}\right) x\right) d \tau \\
+\sum_{i=1}^{m} \int_{t_{i-1}}^{t_{i}}\left(\tilde{B}_{n}^{\prime}(\tau)-B^{\prime}(\tau)\right) A\left(t_{i}\right) x d \tau
\end{gathered}
$$

is less than $3 \varepsilon$ for sufficiently large $n$. Assertion (i) thus follows.

(ii) We fix an arbitrary $W_{*}^{1, \infty}$-derivative $A^{\prime}$ of $A$ and show that $t \mapsto A(t)^{-1} A^{\prime}(t) A(t)^{-1}$ is in $L_{*}^{\infty}(J, L(Y, X))$ and that

$$
A(t)^{-1} y=A(a)^{-1} y-\int_{a}^{t} A(\tau)^{-1} A^{\prime}(\tau) A(\tau)^{-1} y d \tau
$$

for every $t \in J$ and $y \in Y$. As above, it follows from Lemma A 4 of [30] that $t \mapsto$ $A(t)^{-1} A^{\prime}(t) A(t)^{-1}$ is indeed in $L_{*}^{\infty}(J, L(X, Z))$. Since $\sup _{t \in J}\left\|\tilde{A}_{n}(t)-A(t)\right\| \longrightarrow 0$ as $n \rightarrow \infty$, there is an $n_{0} \in \mathbb{N}$ such that $\tilde{A}_{n}(t)$ is invertible (bijective onto $Y$ ) for all $t \in J$ and $n \geq n_{0}$ and such that $\left\|\tilde{A}_{n}(t)^{-1}\right\| \leq \tilde{c}<\infty$ for all $t \in J$ and $n \geq n_{0}$. It follows from the SOT-continuous differentiability of $t \mapsto \tilde{A}_{n}(t)$ that $t \mapsto \tilde{A}_{n}(t)^{-1}$ is SOT-continuously differentiable as well, whence

$$
\tilde{A}_{n}(t)^{-1} y=\tilde{A}_{n}(a)^{-1} y-\int_{a}^{t} \tilde{A}_{n}(\tau)^{-1} \tilde{A}_{n}^{\prime}(\tau) \tilde{A}_{n}(\tau)^{-1} y d \tau
$$


for every $t \in J, y \in Y$ and $n \geq n_{0}$. We now fix $t \in J$ and $y \in Y$. As $\sup _{\tau \in J} \| \tilde{A}_{n}(\tau)^{-1}-$ $A(\tau)^{-1} \| \longrightarrow 0$ as $n \rightarrow \infty$, we obtain

$$
\begin{gathered}
\int_{a}^{t} \tilde{A}_{n}(\tau)^{-1} \tilde{A}_{n}^{\prime}(\tau) \tilde{A}_{n}(\tau)^{-1} y d \tau-\int_{a}^{t} A(\tau)^{-1} A^{\prime}(\tau) A(\tau)^{-1} y d \tau \\
=\int_{a}^{t} \tilde{A}_{n}(\tau)^{-1}\left(\tilde{A}_{n}^{\prime}(\tau) \tilde{A}_{n}(\tau)^{-1} y-A^{\prime}(\tau) A(\tau)^{-1} y\right) d \tau \\
\quad+\int_{a}^{t}\left(\tilde{A}_{n}(\tau)^{-1}-A(\tau)^{-1}\right) A^{\prime}(\tau) A(\tau)^{-1} y d \tau \longrightarrow 0
\end{gathered}
$$

as $n \rightarrow \infty$, where the first term is treated as in the proof of (i). Assertion (ii) thus follows.

We shall need the following simple product rule very often: it will always be used for estimating the difference of two evolution systems and for establishing adiabaticity of evolution systems. And furthermore, it will take the role of Lemma 2.5 in the adiabatic theorems for time-dependent domains (Section 5).

Lemma 2.6. Suppose $C(t)$ is a bounded linear map in $X$ for every $t \in J=[a, b]$, let $t_{0} \in[a, b)$, and let $Y_{t_{0}}$ be a dense subspace of $X$. Suppose that $t \mapsto C(t) y$ is right differentiable at $t_{0}$ for all $y \in Y_{t_{0}}$ and that the map $f: J \rightarrow X$ is right differentiable at $t_{0}$ and $f\left(t_{0}\right) \in Y_{t_{0}}$. Suppose finally that $\sup _{t \in J_{t_{0}}}\|C(t)\|<\infty$ for a neighbourhood $J_{t_{0}}$ of $t_{0}$. Then $t \mapsto C(t) f(t)$ is right differentiable at $t_{0}$ with right derivative

$$
\partial_{+}(C(.) f(.))\left(t_{0}\right)=\partial_{+} C\left(t_{0}\right) f\left(t_{0}\right)+C\left(t_{0}\right) \partial_{+} f\left(t_{0}\right) .
$$

Proof. We have

$$
\begin{aligned}
& \frac{C\left(t_{0}+h\right) f\left(t_{0}+h\right)-C\left(t_{0}\right) f\left(t_{0}\right)}{h} \\
& =C\left(t_{0}+h\right) \frac{f\left(t_{0}+h\right)-f\left(t_{0}\right)}{h}-\frac{C\left(t_{0}+h\right) f\left(t_{0}\right)-C\left(t_{0}\right) f\left(t_{0}\right)}{h}
\end{aligned}
$$

for positive and sufficiently small $h$. Since $\sup _{t \in J_{t_{0}}}\|C(t)\|<\infty$ and $Y_{t_{0}}$ is dense, we easily get that $C\left(t_{0}+h\right) \longrightarrow C\left(t_{0}\right)$ as $h \searrow 0$ w.r.t. SOT, and the desired conclusion follows.

We shall also need the following lemma on the relation between right differentiability and the class $W^{1, \infty}$. It will be used very often - especially in Section 5- in conjunction with the lemma above: Lemma 2.6 will yield right differentiability of a given product and Lemma 2.7, which is a variant of Corollary 2.1.2 of [43], will then yield an integral representation for this product.

Lemma 2.7. Suppose $f: J \rightarrow X$ is a continuous, right differentiable map on a compact interval $J=[a, b]$ such that the right derivative $\partial_{+} f:[a, b) \rightarrow X$ is bounded. Then $f$ is in $W^{1, \infty}(J, X)$ and

$$
f(t)=f\left(t_{0}\right)+\int_{t_{0}}^{t} \partial_{+} f(\tau) d \tau
$$


for all $t_{0}, t \in J$. In particular, if $\partial_{+} f$ is even continuous and continuously extendable to the right endpoint $b$, then $f$ is continuously differentiable.

Proof. Since $\partial_{+} f$ is measurable (as the pointwise limit of a sequence of difference quotients) and $\partial_{+} f$ is bounded, we have only to show that

$$
\int_{(a, b)} f(t) \varphi^{\prime}(t) d t=-\int_{(a, b)} \partial_{+} f(t) \varphi(t) d t \quad \text { for all } \varphi \in C_{c}^{\infty}((a, b), \mathbb{C})
$$

in order to get $f \in W^{1, \infty}(J, X)$ (from which, in turn, the asserted integral representation follows by the continuity of $f)$. So, let $\varphi \in C_{c}^{\infty}((a, b), \mathbb{C})$ and denote by $\tilde{\varphi}$ and $\tilde{f}$ the zero extension of $\varphi$ and $f$ to the whole real line. Then

$$
\begin{aligned}
\int_{(a, b)} f(t) \varphi^{\prime}(t) d t & =\lim _{h \searrow 0} \int_{\mathbb{R}} \tilde{f}(t) \frac{\tilde{\varphi}(t-h)-\tilde{\varphi}(t)}{-h} d t \\
& =-\lim _{h \searrow 0} \int_{\mathbb{R}} \frac{\tilde{f}(t+h)-\tilde{f}(t)}{h} \tilde{\varphi}(t) d t=\int_{(a, b)} \partial_{+} f(t) \varphi(t) d t
\end{aligned}
$$

since $\operatorname{supp} \varphi \subset[a+\delta, b-\delta]$ for some $\delta>0$ and since

$$
\left\|\frac{f(t+h)-f(t)}{h} \varphi(t)\right\| \leq \sup _{\tau \in(a, b)}\left\|\partial_{+} f(\tau)\right\|\|\varphi\|_{\infty}<\infty
$$

for all $t \in[a+\delta, b-\delta]$ and $h \in(0, \delta)$ (which mean value estimate can be derived from the continuity and right differentiability of $f$ in a similar way as Lemma III.1.36 of [31]).

\subsection{Well-posedness and evolution systems}

We briefly recall the fundamental notion of evolution systems for $A$ on $D(A(t))$ where $A$ is a family of linear operators. (See Definition VI.9.2 in [20] which also covers the more general case of evolution systems for $A$ on certain subspaces $Y_{t}$ of $D(A(t))$. We will only work with the special case $Y_{t}=D(A(t))$ since it is only in this case that an adiabatic theory with reasonably practical assumptions can be developed.) Suppose that $A(t): D(A(t)) \subset X \rightarrow X$ is a linear operator for every $t \in J=[a, b]$ and that $U(t, s)$ is a bounded linear operator in $X$ for every $(s, t) \in \Delta_{J}:=\left\{(s, t) \in J^{2}: s \leq t\right\}$. Then $U$ is called an evolution system for $A$ on (the spaces) $D(A(t))$ if and only if the following holds true:

(i) $[s, b] \ni t \mapsto U(t, s) y$ is a continuously differentiable solution to the initial value problem $x^{\prime}=A(t) x, x(s)=y$ for $y \in D(A(s))$ and $s \in[a, b)$

(ii) $U(t, s) U(s, r)=U(t, r)$ for all $(r, s),(s, t) \in \Delta_{J}$ and $\Delta_{J} \ni(s, t) \mapsto U(t, s) x$ is continuous for all $x \in X$.

We refer to [20] (Definition VI.9.1) for the definition of well-posedness of the initial value problems corresponding to $A$ on $D(A(t))$. It is well-known that there is a one-toone correspondence between well-posedness and evolution systems (Proposition VI.9.3 
of [20]): if $A$ is a family of linear operators $A(t): D(A(t)) \subset X \rightarrow X$, then the initial value problems corresponding to $A$ are well-posed on $D(A(t))$ if and only if there is an evolution system $U$ for $A$ on $D(A(t))$. In particular, it follows (by the uniqueness requirement in the definition of well-posedness) that if there is any evolution system for a given family $A$ on $D(A(t))$, then it is already unique. In order to see the above-mentioned correspondence one essentially has only to combine Lemma 2.6 and Lemma 2.7 with the following simple lemma, which will always be used when the difference of two evolution systems has to be dealt with.

Lemma 2.8. Suppose $A(t): D(A(t)) \subset X \rightarrow X$ for every $t \in J=[a, b]$ is a densely defined linear map and suppose there is an evolution system $U$ for $A$ on $D(A(t))$. Then, for every $s_{0} \in[a, t)$ and every $x_{0} \in D\left(A\left(s_{0}\right)\right)$, the map $[a, t] \ni s \mapsto U(t, s) x_{0}$ is right differentiable at $s_{0}$ with right derivative $-U\left(t, s_{0}\right) A\left(s_{0}\right) x_{0}$. In particular, if $D(A(t))=D$ for all $t \in J$ and $s \mapsto A(s) x$ is continuous for all $x \in D$, then $[a, t] \ni s \mapsto U(t, s) x$ is continuously differentiable for all $x \in D$.

Proof. Since $U(t, s) U(s, r)=U(t, r)$ for $(r, s),(s, t) \in \Delta_{J}$ and since $\Delta_{J} \ni(s, t) \mapsto U(t, s)$ is SOT-continuous, we obtain for every $s_{0} \in[a, t)$ and $x_{0} \in D\left(A\left(s_{0}\right)\right)$ that

$$
\frac{U\left(t, s_{0}+h\right) x_{0}-U\left(t, s_{0}\right) x_{0}}{h}=-U\left(t, s_{0}+h\right) \frac{U\left(s_{0}+h, s_{0}\right) x_{0}-x_{0}}{h} \longrightarrow-U\left(t, s_{0}\right) A\left(s_{0}\right) x_{0}
$$

as $h \searrow 0$ from which the assertions follow (remember Lemma 2.7).

We also briefly recall the notion of $(M, \omega)$-stability from [29]: a family $A$ of linear operators $A(t): D(A(t)) \subset X \rightarrow X$ (where $t \in J$ ) is called $(M, \omega)$-stable (for some $M \in[1, \infty)$ and $\omega \in \mathbb{R}$ ) if and only if $A(t)$ generates a strongly continuous semigroup on $X$ for every $t \in J$ and

$$
\left\|e^{A\left(t_{n}\right) s_{n}} \cdots e^{A\left(t_{1}\right) s_{1}}\right\| \leq M e^{\omega\left(s_{1}+\cdots+s_{n}\right)}
$$

for all $s_{1}, \ldots, s_{n} \in[0, \infty)$ and all $t_{1}, \ldots, t_{n} \in J$ satisfying $t_{1} \leq \cdots \leq t_{n}$ (with arbitrary $n \in$ $\mathbb{N}$ ). Clearly, a family $A$ of linear operators in $X$ is $(1,0)$-stable if and only if each member $A(t)$ of the family generates a contraction semigroup on $X$. It should be remarked that there are very simple examples - relevant to adiabatic theory - of $(M, 0)$-stable families that fail to be $(1,0)$-stable (Example 3.3). When it comes to estimating perturbed evolution systems in Section 3 and 4, the following important fact (well-known from [29]) will always - and tacitly - be used: if $A$ is an $(M, \omega)$-stable family of linear operators $A(t): D(A(t)) \subset X \rightarrow X$ for $t \in J, B(t)$ is a bounded operator in $X$ for $t \in J$ and $b:=\sup _{t \in J}\|B(t)\|$ is finite, then $A+B$ is $(M, \omega+M b)$-stable. In our examples the following lemma will be important.

Lemma 2.9. Suppose $A_{0}$ is an $\left(M_{0}, \omega_{0}\right)$-stable family of operators $A_{0}(t): D\left(A_{0}(t)\right) \subset$ $X \rightarrow X$ for $t \in J$ and $R(t): X \rightarrow X$ for every $t \in J$ is a bijective bounded operator such that $t \mapsto R(t)$ is in $W_{*}^{1, \infty}(J, L(X))$. Then the family $A$ with $A(t):=R(t)^{-1} A_{0}(t) R(t)$ is $(M, \omega)$-stable for some $M \in[1, \infty)$ and $\omega=\omega_{0}$. 
Proof. We may assume that $\omega_{0}=0$, since $(\tilde{M}, \tilde{\omega})$-stability of a family $\tilde{A}$ is equivalent to the $(\tilde{M}, 0)$-stability of $\tilde{A}-\tilde{\omega}$. Set $\|x\|_{t}:=d e^{-M_{0} c t}\|R(t) x\|_{0 t}$ for $x \in X$ and $t \in J$, where $c:=\operatorname{ess}_{-\sup _{(s, t) \in J^{2}}}\left\|R^{\prime}(t) R(s)^{-1}\right\|$ and $d:=\sup _{t \in J} e^{M_{0} c t}\left\|R(t)^{-1}\right\|$ and the $\|\cdot\|_{0 t}$ are norms on $X$ associated with $A_{0}$ according to Proposition 1.3 of [42]. Then - as can be gathered from the proof of Theorem 4.2 in [34] - the norms $\|\cdot\|_{t}$ satisfy the conditions (a), (b), (c) of Proposition 1.3 in [42] for the family $A$ with a certain $M \in[1, \infty)$ and therefore $A$ is $(M, 0)$-stable, as desired.

We now turn to sufficient conditions for the well-posedness of the initial value problems corresponding to a given family $A$ of linear operators $A(t)$. We will make very much use of the following condition.

Condition 2.10. $A(t): D \subset X \rightarrow X$ for every $t \in I$ is a densely defined closed linear map such that $A$ is $(M, \omega)$-stable for some $M \in[1, \infty)$ and $\omega \in \mathbb{R}$ and such that $t \mapsto A(t)$ is in $W_{*}^{1, \infty}(I, L(Y, X))$, where $Y$ is the space $D$ endowed with the graph norm of $A(0)$.

As was noted (in Proposition 2.4) above, the $W_{*}^{1, \infty}$-regularity requirement of Condition 2.10 is fulfilled if, for instance, $t \mapsto A(t)$ is SOT- or WOT-continuously differentiable. It follows from a famous theorem of Kato (Theorem 1 of [30]) - and, for seperable spaces, is explicitly remarked in Section 1 of [32] - that Condition 2.10 guarantees well-posedness.

Theorem 2.11 (Kato). Suppose $A(t): D \subset X \rightarrow X$ for every $t \in I$ is a linear map such that Condition 2.10 is satisfied. Then there is a unique evolution system $U$ for $A$ on $D$, and the following estimate holds true:

$$
\|U(t, s)\| \leq M e^{\omega(t-s)} \text { for all }(s, t) \in \Delta .
$$

Condition 2.10 does not only guarantee well-posedness, but it is also essentially everything we have to require of $A$ in the adiabatic theorems of Section 3 and 4 for timeindependent domains: indeed, we have only to add the requirement that $\omega=0$ to Condition 2.10 to arrive at the hypotheses on $A$ of these theorems. In most adiabatic theorems in the literature - for example those of [9], [7], [52], [53], [1], [2] or [8] - by contrast, the hypotheses on $A$ rest upon Yosida's theorem (Theorem XIV.4.1 of [54]), which is reproduced, for instance, in Reed and Simon's book (Theorem X.70 of [45]) or Blank, Exner and Havlíček's book (Theorem 9.5.3 of [11]): in these adiabatic theorems it is required of $A$ that each $A(t)$ generate a contraction semigroup on $X$ and that an appropriate translate $A-z_{0}$ of $A$ satisfy the rather involved hypotheses of Yosida's theorem (or - for example in the case of [9] or [8] - more convenient strengthenings thereof). It is shown in [47] that this is the case if and only if $A(t)-z_{0}$, for every $t \in I$, is a boundedly invertible generator of a contraction semigroup on $X$ and

$$
t \mapsto A(t) x \text { is continuously differentiable for all } x \in D \text {. }
$$

In particular, it follows that the hypotheses on $A$ of the adiabatic theorems of the present paper are more general than the respective hypotheses of the previously known adiabatic theorems - and, of course, they are also striclty more general (which is demonstrated by the examples of Section 3 and 4). 
A trivial - but nonetheless useful - consequence of Theorem 2.11 is the following corollary establishing well-posedness for families $A$ where $A(t)=R(t)^{-1} A_{0}(t) R(t)$ and $D\left(A_{0}(t)\right)$ is time-independent. A non-trivial sufficient condition for well-posedness in the case of time-dependent domains is furnished, for instance, by [34] (which will be exploited in Section 6) or by [49], 28], 21], 44.

Corollary 2.12. Suppose $A_{0}$ is a family of linear maps $A_{0}(t): D \subset X \rightarrow X$ that satisfies Condition 2.10 and let $A(t):=R(t)^{-1} A_{0}(t) R(t)$ for $t \in I$, where $t \mapsto R(t)$ is in $W_{*}^{2, \infty}(I, L(X))$ and $R(t)$ is bijective onto $X$ for every $t \in I$. Then there is a unique evolution system $U$ for $A$ on $D(A(t))$.

Proof. Since $t \mapsto A_{0}(t)+R^{\prime}(t) R(t)^{-1}$ is in $W_{*}^{1, \infty}(I, L(Y, X))$ by Lemma 2.5 and since $A_{0}+R^{\prime} R^{-1}$ is $(M, \omega+M b)$-stable with $b:=\sup _{t \in I}\left\|R^{\prime}(t) R(t)^{-1}\right\|$, it follows from Theorem 2.11 that there is a unique evolution system $\tilde{U}_{0}$ for $A_{0}+R^{\prime} R^{-1}$ on $D$. Set $U(t, s):=R(t)^{-1} \tilde{U}_{0}(t, s) R(s)$ for $(s, t) \in \Delta$. Then $U$ is an evolution system for $A$ on $D(A(t))$, as is easily verified.

In the adiabatic theorems with spectral gap condition of Section 5 (especially in the adiabatic theorem of higher order) the following well-expected perturbative proposition will be needed. It gives a perturbation series expansion for a perturbed evolution system if only this perturbed evolution exists. (See the classical example of Phillips (Example 6.4 of [44]) showing that the existence of the perturbed evolution really has to be required.)

Proposition 2.13. Suppose that $A(t): D(A(t)) \subset X \rightarrow X$ is a densely defined linear map for every $t \in I$ and that $t \mapsto B(t) \in L(X)$ is WOT-continuous. Suppose further that there is an evolution system $U$ for $A$ on $D(A(t))$ and an evolution system $V$ for $A+B$ on $D(A(t))$. Then

(i) $V(t, s)=\sum_{n=0}^{\infty} V_{n}(t, s)$, where $V_{0}(t, s):=U(t, s)$ and

$$
V_{n+1}(t, s) x:=\int_{s}^{t} U(t, \tau) B(\tau) V_{n}(\tau, s) x d \tau \text { for } x \in X \text { and } n \in \mathbb{N} \cup\{0\} .
$$

(ii) If there are $M \in[1, \infty), \omega \in \mathbb{R}$ such that $\|U(t, s)\| \leq M e^{\omega(t-s)}$ for $(s, t) \in \Delta$, then

$$
\|V(t, s)\| \leq M e^{(\omega+M b)(t-s)}
$$

for all $(s, t) \in \Delta$, where $b:=\sup _{t \in I}\|B(t)\|$. And if, for every $(s, t) \in \Delta, U(t, s)$ is unitary and $B(t)$ is skew symmetric, then $V(t, s)$ is unitary as well.

Proof. Since weakly continuous maps on compact intervals are integrable (see the proof of Proposition 2.4), it easily follows that the integrals defining the $V_{n}$ really exist and that $\tilde{V}(t, s):=\sum_{n=0}^{\infty} V_{n}(t, s)$ exists uniformly in $(s, t) \in \Delta$. Also, it is easy to see - applying Lemma 2.6 and Lemma 2.7 to $[s, t] \ni \tau \mapsto U(t, \tau) V(\tau, s) x$ with $x \in D(A(s))$ - that $V$ satisfies the same integral equation as $\tilde{V}$ from which assertion (i) follows. Assertion (ii) is a simple consequence of the series expansion in (i). 


\subsection{Adiabatic evolutions and a trivial adiabatic theorem}

As has been explained in Section 1, the principal goal of adiabatic theory is to establish the convergence (1.1) or, in other words, to show that the evolution systems $U_{\varepsilon}$ for $\frac{1}{\varepsilon} A$ are, in some sense, approximately adiabatic w.r.t. $P$ as $\varepsilon \searrow 0$. We say that an evolution system for a family $A$ of linear operators $A(t): D(A(t)) \subset X \rightarrow X$ is adiabatic w.r.t. a family $P$ of bounded projections $P(t)$ in $X$ if and only if $U(t, s)$ for every $(s, t) \in \Delta$ intertwines $P(s)$ with $P(t)$, more precisely:

$$
P(t) U(t, s)=U(t, s) P(s)
$$

for every $(s, t) \in \Delta$. Since the pioneering work [26] of Kato, the basic strategy in proving the convergence (1.1) has been to show that

$$
U_{\varepsilon}(t)-V_{\varepsilon}(t) \longrightarrow 0 \quad(\varepsilon \searrow 0)
$$

for every $t \in I$, where the $V_{\varepsilon}$ are suitable comparison evolution systems that are adiabatic w.r.t. the family $P$ of projections $P(t)$ related to the data $A, \sigma$. A simple way of obtaining adiabatic evolutions w.r.t. some given family $P$ (independently observed by Kato in [26] and Daleckii-Krein in [16]) is described in the following important proposition.

Proposition 2.14 (Kato, Daleckii-Krein). Suppose $A(t): D(A(t)) \subset X \rightarrow X$ for every $t \in I$ is a densely defined closed linear map and $P(t)$ a bounded projection in $X$ such that $P(t) A(t) \subset A(t) P(t)$ for every $t \in I$ and $t \mapsto P(t)$ is SOT-continuously differentiable. If the evolution system $V_{\varepsilon}$ for $\frac{1}{\varepsilon} A+\left[P^{\prime}, P\right]$ exists on $D(A(t))$ for every $\varepsilon \in(0, \infty)$, then $V_{\varepsilon}$ is adiabatic w.r.t. $P$ for every $\varepsilon \in(0, \infty)$.

Proof. Choose an arbitrary $(s, t) \in \Delta$ with $s \neq t$. It then follows by Lemma 2.6 and Lemma 2.8 that, for every $x \in D(A(s))$, the map

$$
[s, t] \ni \tau \mapsto V_{\varepsilon}(t, \tau) P(\tau) V_{\varepsilon}(\tau, s) x
$$

is continuous and right differentiable. Since $P(\tau)$ commutes with $A(\tau)$ and

$$
P(\tau) P^{\prime}(\tau) P(\tau)=0
$$

for every $\tau \in I$ (which follows by applying $P$ from the left and the right to $P^{\prime}=$ $\left.P^{\prime} P+P P^{\prime}\right)$, it further follows that the right derivative of this map is identically 0 and so (by Lemma 2.7) this map is constant. In particular,

$$
P(t) V_{\varepsilon}(t, s) x-V_{\varepsilon}(t, s) P(s) x=\left.V_{\varepsilon}(t, \tau) P(\tau) V_{\varepsilon}(\tau, s) x\right|_{\tau=s} ^{\tau=t}=0,
$$

as desired.

We now briefly discuss two situations where the conclusion of the adiabatic theorem is already trivially true. 
Proposition 2.15. Suppose $A(t): D(A(t)) \subset X \rightarrow X$ for every $t \in I$ is a densely defined closed linear map and $P(t)$ is a bounded projection in $X$ such that the evolution system $U_{\varepsilon}$ exists on $D(A(t))$ for every $\varepsilon \in(0, \infty)$ and such that $P(t) A(t) \subset A(t) P(t)$ for every $t \in I$ and $t \mapsto P(t)$ is SOT-continuously differentiable.

(i) If $P^{\prime}=0$, then $U_{\varepsilon}$ is adiabatic w.r.t. $P$ for every $\varepsilon \in(0, \infty)$ (in particular, the convergence (1.1) holds trivially), and the reverse implication is also true.

(ii) If there are $\gamma \in(0, \infty)$ and $M \in[1, \infty)$ such that for all $(s, t) \in \Delta$ and $\varepsilon \in(0, \infty)$

$$
\left\|U_{\varepsilon}(t, s)\right\| \leq M e^{-\frac{\gamma}{\varepsilon}(t-s)},
$$

then $\sup _{t \in I}\left\|U_{\varepsilon}(t)-V_{\varepsilon}(t)\right\|=O(\varepsilon)$ as $\varepsilon \searrow 0$, whenever the evolution system $V_{\varepsilon}$ for $\frac{1}{\varepsilon} A+\left[P^{\prime}, P\right]$ exists on $D(A(t))$ for every $\varepsilon \in(0, \infty)$.

Proof. (i) See, for instance, Section IV.3.2 of [35] for the reverse implication (differentiate the adiabaticity relation with respect to the variable $s$ ) - the other implication is obvious from Proposition 2.14.

(ii) Since for $x \in D(A(0))$ one has (by Lemma 2.8, Lemma 2.6, and Lemma 2.7)

$$
V_{\varepsilon}(t) x-U_{\varepsilon}(t) x=\left.U_{\varepsilon}(t, s) V_{\varepsilon}(s) x\right|_{s=0} ^{s=t}=\int_{0}^{t} U_{\varepsilon}(t, s)\left[P^{\prime}(s), P(s)\right] V_{\varepsilon}(s) x d s
$$

for every $t \in I$ and $\varepsilon \in(0, \infty)$, it follows with the help of Proposition 2.13 that

$$
\left\|U_{\varepsilon}(t)-V_{\varepsilon}(t)\right\| \leq M^{2} c e^{M c} t e^{-\frac{\gamma}{\varepsilon} t}
$$

for all $t \in I$ and $\varepsilon \in(0, \infty)$, where $c$ denotes an upper bound of $s \mapsto\left\|\left[P^{\prime}(s), P(s)\right]\right\|$. And from this the desired conclusion is obvious.

Combining Proposition 2.15 (ii) with Example 3.5 one sees that adiabatic theory is interesting only if the evolution systems for $\frac{1}{\varepsilon} A$ are only just bounded w.r.t. $\varepsilon \in(0, \infty)$ : if even the evolution for $\frac{1}{\varepsilon}(A+\gamma)$ is bounded in $\varepsilon \in(0, \infty)$ for some $\gamma>0$, then adiabatic theory is trivial for $A$ (by Proposition 2.15 (ii)), and if only the evolution for $T(A-\gamma)$ is bounded in $\varepsilon \in(0, \infty)$ for some $\gamma>0$, then adiabatic theory is generally impossible for $A$ (by Example 3.5 ).

\subsection{Standard examples}

We will complement the adiabatic theorems of this paper by examples in order to demonstrate, on the one hand, that the presented theorems are strictly more general than the previously known adiabatic theorems (positive examples) and that, on the other hand, some selected hypotheses of our theorems cannot be dispensed with (negative examples). We have made sure that in all positive examples the conclusion of the respective adiabatic theorem is not already trivially fulfilled in the sense that it does not already follow from the trivial adiabatic theorem presented above. (See Example 3.3 where this is once - and for all - explained in detail.) All examples will be of the following simple standard structure: 
- $X=\ell^{p}\left(I_{d}\right)$ for some $p \in\left[1, \infty\right.$ ) and $d \in \mathbb{N} \cup\{\infty\}$ (where $I_{d}:=\{1, \ldots, d\}$ for $d \in \mathbb{N}$ and $\left.I_{\infty}:=\mathbb{N}\right)$ or $X=L^{p}\left(X_{0}\right)$ for some $p \in[1, \infty)$ and some measure space $\left(X_{0}, \mathcal{A}, \mu\right)$ or $X$ is a product of some of the aforementioned spaces (endowed with the sum norm)

- $A(t)=R(t)^{-1} A_{0}(t) R(t)$, where $A_{0}(t): D \subset X \rightarrow X$ is a semigroup generator on $X$ with $t$-independent dense domain $D$ (chosen equal or unequal to $X$ depending on whether we are in the case of time-independent or time-dependent domains), $A_{0}$ satisfies Condition 2.10, and $R(t):=e^{C t}$ for some bounded operator $C$.

Condition 2.10 with $\omega=0$ for $A_{0}$ ensures (by Lemma 2.9 and Corollary 2.12) that the hypotheses on $A$ of the adiabatic theorems of Sections 3 to 5 are fulfilled. In some of our examples we will use the right or left shift operator $S_{+}$and $S_{-}$on $\ell^{p}\left(I_{\infty}\right)$ defined by

$$
S_{+}\left(x_{1}, x_{2}, \ldots\right):=\left(0, x_{1}, x_{2}, \ldots\right) \text { and } S_{-}\left(x_{1}, x_{2}, x_{3}, \ldots\right):=\left(x_{2}, x_{3}, \ldots\right) .
$$

Since $\left\|S_{ \pm}\right\| \leq 1$, it follows from the theorem of Hille-Yosida that $e^{i \vartheta} S_{+}-1$ and $e^{i \vartheta} S_{-}-1$ generate contraction semigroups on $\ell^{p}\left(I_{\infty}\right)$ for $p \in[1, \infty)$ and $\vartheta \in \mathbb{R}$ (use a Neumann series expansion!). It is well-known (Example V.4.1 and V.4.2 of [51]) that $\sigma\left(S_{ \pm}\right)=\bar{U}_{1}(0)$ for all $p \in[1, \infty)$, the fine structure of $\sigma\left(S_{+}\right)$being given by

$$
\begin{aligned}
& \sigma_{p}\left(S_{+}\right)=\emptyset, \quad \sigma_{c}\left(S_{+}\right)=\emptyset, \quad \sigma_{r}\left(S_{+}\right)=\bar{U}_{1}(0) \quad(p=1) \\
& \sigma_{p}\left(S_{+}\right)=\emptyset, \quad \sigma_{c}\left(S_{+}\right)=\partial U_{1}(0), \quad \sigma_{r}\left(S_{+}\right)=U_{1}(0) \quad(p \in(1, \infty))
\end{aligned}
$$

and the fine structure of $\sigma\left(S_{-}\right)$being given by

$$
\sigma_{p}\left(S_{-}\right)=U_{1}(0), \quad \sigma_{c}\left(S_{-}\right)=\partial U_{1}(0), \quad \sigma_{r}\left(S_{-}\right)=\emptyset \quad(p \in[1, \infty)) .
$$

Additionally, we will sometimes use multiplication operators $M_{f}$ on $L^{p}\left(X_{0}\right)(p \in[1, \infty))$ for some measurable function $f: X_{0} \rightarrow \mathbb{C}$ and some $\sigma$-finite measure space $\left(X_{0}, \mathcal{A}, \mu\right)$ in which case, as is well-known (Proposition I.4.10 of [20]), one has

$$
\sigma\left(M_{f}\right)=\operatorname{ess-rg} f:=\left\{z \in \mathbb{C}: \mu\left(f^{-1}\left(U_{\varepsilon}(z)\right)\right) \neq 0 \text { for all } \varepsilon>0\right\}
$$

and, in particular (take $\mu$ to be the counting measure on $X_{0}:=I_{d}$ ),

$$
\sigma\left(\operatorname{diag}\left(\left(\lambda_{n}\right)_{n \in I_{d}}\right)\right)=\sigma\left(M_{\left(\lambda_{n}\right)_{n \in I_{d}}}\right)=\overline{\left\{\lambda_{n}: n \in I_{d}\right\}} .
$$

In quite some examples, we will work with families $A$ of operators $A(t)$ in $X:=$ $\ell^{p}\left(I_{d}\right)$ whose spectra $\sigma(A(t))$ are singletons and whose nilpotent parts depend on $t$ in the simplest possible way, namely via a scalar factor.

Condition 2.16. $N \neq 0$ is a nilpotent operator in $X:=\ell^{p}\left(I_{d}\right)$ (with $p \in[1, \infty)$ and $d \in \mathbb{N}), \lambda(t) \in \mathbb{C}$ and $\alpha(t) \in[0, \infty)$ for all $t \in I$, and there is an $r_{0}>0$ such that $-\operatorname{Re} \lambda(t)=|\operatorname{Re} \lambda(t)| \geq r_{0} \alpha(t)$ for all $t \in I$.

As is shown in the next lemma, this condition characterizes $(M, 0)$-stability of families $A$ of the simple type just described. 
Lemma 2.17. Suppose that $N \neq 0$ is a nilpotent operator in $X:=\ell^{p}\left(I_{d}\right)$ with $p \in[1, \infty)$ and $d \in \mathbb{N}$ and that $A(t)=\lambda(t)+\alpha(t) N$ for every $t \in I$, where $\lambda(t) \in \mathbb{C}$ and $\alpha(t) \in[0, \infty)$. Then $A$ is $(M, 0)$-stable for some $M \in[1, \infty)$ if and only if Condition [2.16 is satisfied.

Proof. Suppose first that $A$ is $(M, 0)$-stable for some $M \in[1, \infty)$ and assume that $N=\operatorname{diag}\left(J_{1}, \ldots, J_{m}\right)$ is in Jordan normal form with (decreasingly ordered) Jordan block matrices $J_{1}, \ldots, J_{m}$ (notice that this assumption, by virtue of Lemma 2.9, does not restrict generality). We then show that $-\operatorname{Re} \lambda(t)=|\operatorname{Re} \lambda(t)| \geq \frac{1}{4 M} \alpha(t)$ for every $t \in I$. It is clear by the $(M, 0)$-stability of $A$ that $\lambda(t) \in \sigma(A(t)) \subset\{\operatorname{Re} z \leq 0\}$ for every $t \in I$ and that the family $\tilde{A}$ with $\tilde{A}(t):=\operatorname{Re} \lambda(t)+\alpha(t) N$ is $(M, 0)$-stable as well. If $\alpha(t)=0$, then the desired inequality is trivial. If $\alpha(t) \neq 0$, then $\operatorname{Re} \lambda(t)<0$ by the $(M, 0)$-stability of $A$ and therefore we get - computing $(\lambda-\tilde{A}(t))^{-1} e_{2}=\left(\frac{\alpha(t)}{(\lambda-\operatorname{Re} \lambda(t))^{2}}, \frac{1}{\lambda-\operatorname{Re} \lambda(t)}, 0,0, \ldots\right)$ for $\lambda \in(0, \infty)$, setting $\lambda:=|\operatorname{Re} \lambda(t)|$, and using the $(M, 0)$-stability of $\tilde{A}-$ that

$$
\frac{\alpha(t)}{4|\operatorname{Re} \lambda(t)|} \leq\left\||\operatorname{Re} \lambda(t)|(|\operatorname{Re} \lambda(t)|-\tilde{A}(t))^{-1} e_{2}\right\| \leq M,
$$

as desired. Suppose conversely that there is an $r_{0}>0$ such that $-\operatorname{Re} \lambda(t)=|\operatorname{Re} \lambda(t)| \geq$ $r_{0} \alpha(t)$ for every $t \in I$. Then there is an $M=M_{r_{0}} \in[1, \infty)$ such that $\left\|e^{N s}\right\| \leq M e^{r_{0} s}$ for all $s \in[0, \infty)$ and thus

$$
\left\|e^{A\left(t_{n}\right) s_{n}} \cdots e^{A\left(t_{1}\right) s_{1}}\right\|=e^{\operatorname{Re} \lambda\left(t_{n}\right) s_{n}} \cdots e^{\operatorname{Re} \lambda\left(t_{1}\right) s_{1}}\left\|e^{N\left(\alpha\left(t_{n}\right) s_{n}+\cdots+\alpha\left(t_{1}\right) s_{1}\right)}\right\| \leq M
$$

for all $s_{1}, \ldots, s_{n} \in[0, \infty)$ and all $t_{1}, \ldots, t_{n} \in I$ satisfying $t_{1} \leq \cdots \leq t_{n}$ (with arbitrary $n \in$ $\mathbb{N})$, as desired.

It should be noticed that Condition 2.16 does not already guarantee $(1,0)$-stability, however. Indeed, if for instance

$$
A(t):=-\frac{t}{3}+t^{2} N \quad \text { with } \quad N:=\left(\begin{array}{cccc}
0 & 1 & & \\
& 0 & 1 & \\
& & \ddots & \ddots \\
& & & 0
\end{array}\right) \quad \text { in } X:=\ell^{p}\left(I_{d}\right)
$$

$(p \in[1, \infty)$ and $2 \leq d \in \mathbb{N})$, then $A$ is $(M, 0)$-stable for some $M \in[1, \infty)$ by the above lemma, but not $(1,0)$-stable, because $A(1)=-\frac{1}{3}+N$ is not dissipative in $\ell^{p}\left(I_{d}\right)$ and hence (by the theorem of Lumer-Phillips) does not generate a contraction semigroup.

At some point (Example 4.8) the following simple lemma will be needed which, in essence, is the reason why adiabatic theory for multiplication operators $A(t)=M_{f_{t}}$ is typically uninteresting. See [46] (Lemma 2.11 and the remark following it) for the proof.

Lemma 2.18. Suppose that $P(t)$ for every $t \in I$ is a bounded projection in $X:=L^{p}\left(X_{0}\right)$ (where $\left(X_{0}, \mathcal{A}, \mu\right)$ is a measure space and $p \in[1, \infty)$ ) and that $P(t)=M_{\chi_{E_{t}}}$ for almost every $t \in I$, where $E_{t} \in \mathcal{A}$. If $t \mapsto P(t)$ is SOT-continuously differentiable, then $t \mapsto P(t)$ is already constant. 


\section{Adiabatic theorems with spectral gap condition for time-independent domains}

After having provided the most important preliminaries in Section 2, we now prove an adiabatic theorem with uniform spectral gap condition (Section 3.1) and an adiabatic theorem with non-uniform spectral gap condition (Section 3.2) for general operators $A(t)$ with time-independent domains. In these theorems the considered spectral subsets $\sigma(t)$ are only assumed to be compact so that, even if they are singletons, they need not consist of eigenvalues: they are allowed to be singletons consisting of essential singularities of the resolvent. In [2], 8], 25] the case of poles is treated.

\subsection{An adiabatic theorem with uniform spectral gap condition}

We begin by proving an adiabatic theorem with uniform spectral gap condition by extending Abou Salem's proof from [2], which rests upon solving a suitable commutator equation.

Theorem 3.1. Suppose $A(t): D \subset X \rightarrow X$ for every $t \in I$ is a linear map such that Condition 2.10 is satisfied with $\omega=0$. Suppose further that $\sigma(t)$ for every $t \in I$ is a compact subset of $\sigma(A(t))$, that $\sigma($.$) at no point falls into \sigma(A().) \backslash \sigma($.$) , and that$ $t \mapsto \sigma(t)$ is continuous. And finally, for every $t \in I$, let $P(t)$ be the projection associated with $A(t)$ and $\sigma(t)$ and suppose that $I \ni t \mapsto P(t)$ is in $W_{*}^{2, \infty}(I, L(X))$. Then

$$
\sup _{t \in I}\left\|U_{\varepsilon}(t)-V_{\varepsilon}(t)\right\|=O(\varepsilon) \quad(\varepsilon \searrow 0),
$$

where $V_{\varepsilon}$ is the evolution system for $\frac{1}{\varepsilon} A+\left[P^{\prime}, P\right]$.

Proof. Since $\sigma($.$) is uniformly isolated in \sigma(A().) \backslash \sigma($.$) and t \mapsto \sigma(t)$ is continuous, there is, for every $t_{0} \in I$, a non-trivial closed interval $J_{t_{0}} \subset I$ containing $t_{0}$ and a cycle $\gamma_{t_{0}}$ in $\rho\left(A\left(t_{0}\right)\right)$ such that $\operatorname{rg} \gamma_{t_{0}} \subset \rho(A(t))$ and

$$
\mathrm{n}\left(\gamma_{t_{0}}, \sigma(t)\right)=1 \quad \text { and } \mathrm{n}\left(\gamma_{t_{0}}, \sigma(A(t)) \backslash \sigma(t)\right)=0
$$

for all $t \in J_{t_{0}}$. We can now define

$$
B(t) x:=\frac{1}{2 \pi i} \int_{\gamma_{t_{0}}}(z-A(t))^{-1} P^{\prime}(t)(z-A(t))^{-1} x d z
$$

for all $t \in J_{t_{0}}, t_{0} \in I$ and $x \in X$. Since $\rho(A(t)) \ni z \mapsto(z-A(t))^{-1} P^{\prime}(t)(z-A(t))^{-1} x$ is a holomorphic $X$-valued map (for all $x \in X$ ) and since the cycles $\gamma_{t_{0}}$ and $\gamma_{t_{0}^{\prime}}$ are homologous in $\rho(A(t))$ whenever $t$ lies both in $J_{t_{0}}$ and in $J_{t_{0}^{\prime}}$, the path integral exists in $X$ and does not depend on the special choice of $t_{0} \in I$ with the property that $t \in J_{t_{0}}$. In other words, $t \mapsto B(t)$ is well-defined on $I$.

As a first preparatory step, we easily infer from the closedness of $A(t)$ that $B(t) X \subset$ $D(A(t))=D=Y$ and that

$$
B(t) A(t)-A(t) B(t) \subset\left[P^{\prime}(t), P(t)\right]
$$


for all $t \in I$, which commutator equation will be essential in the main part of the proof. As a second preparatory step, we show that $t \mapsto B(t)$ is in $W_{*}^{1, \infty}(I, L(X, Y))$, which is not very surprising (albeit a bit technical). It suffices to show that $J_{t_{0}} \ni t \mapsto B(t)$ is in $W_{*}^{1, \infty}\left(J_{t_{0}}, L(X, Y)\right)$ for every $t_{0} \in I$. We therefore fix $t_{0} \in I$. Since $\rho(A(t)) \ni$ $z \mapsto(z-A(t))^{-1}$ is continuous w.r.t. the norm of $L(X, Y)$ for every $t \in J_{t_{0}}$, we see that $B(t)$ is in $L(X, Y)$ for every $t \in J_{t_{0}}$. We also see, by virtue of Lemma 2.5, that for every $z \in \operatorname{rg} \gamma_{t_{0}}$ the map $t \mapsto(z-A(t))^{-1} P^{\prime}(t)(z-A(t))^{-1}$ is in $W_{*}^{1, \infty}\left(J_{t_{0}}, L(X, Y)\right)$ and $t \mapsto C(t, z)=C_{1}(t, z)+C_{2}(t, z)+C_{3}(t, z)$ is a $W_{*}^{1, \infty}$-derivative of it, where

$$
\begin{gathered}
C_{1}(t, z)=(z-A(t))^{-1} A^{\prime}(t)(z-A(t))^{-1} P^{\prime}(t)(z-A(t))^{-1}, \\
C_{2}(t, z)=(z-A(t))^{-1} P^{\prime \prime}(t)(z-A(t))^{-1} \\
C_{3}(t, z)=(z-A(t))^{-1} P^{\prime}(t)(z-A(t))^{-1} A^{\prime}(t)(z-A(t))^{-1}
\end{gathered}
$$

and $A^{\prime}, P^{\prime \prime}$ are arbitrary $W_{*}^{1, \infty}$-derivatives of $A$ and $P^{\prime}$. Since $t \mapsto C(t, z)$ is strongly measurable for all $z \in \operatorname{rg} \gamma_{t_{0}}$, it follows that $t \mapsto \frac{1}{2 \pi i} \int_{\gamma_{t_{0}}} C(t, z) d z$ is strongly measurable as well (as the strong limit of Riemann sums), and since $J_{t_{0}} \times \operatorname{rg} \gamma_{t_{0}} \ni(t, z) \mapsto(z-A(t))^{-1}$ is continuous w.r.t. the norm of $L(X, Y)$ and hence bounded, it follows by (3.2) that

$$
t \mapsto\left\|\frac{1}{2 \pi i} \int_{\gamma_{t_{0}}} C(t, z) d z\right\|_{X \rightarrow Y}
$$

is essentially bounded. So $t \mapsto \frac{1}{2 \pi i} \int_{\gamma_{t_{0}}} C(t, z) d z$ is in $W_{*}^{0, \infty}\left(J_{t_{0}}, L(X, Y)\right)$ and one easily concludes that

$$
B(t) x=B\left(t_{0}\right) x+\int_{t_{0}}^{t} \frac{1}{2 \pi i} \int_{\gamma_{t_{0}}} C(\tau, z) x d z d \tau
$$

for all $t \in J_{t_{0}}$ and $x \in X$, as desired.

After these preparations we can now turn to the main part of the proof. We fix $x \in D$ and let $V_{\varepsilon}$ denote the evolution system for $\frac{1}{\varepsilon} A+\left[P^{\prime}, P\right]$ (which really exists due to Theorem 2.11). Then $s \mapsto U_{\varepsilon}(t, s) V_{\varepsilon}(s) x$ is in $W^{1, \infty}([0, t], X)$ (by Lemma 2.6 and Lemma 2.7) and we get, exploiting the commutator equation (3.1) for $A$ and $B$, that

$$
\begin{aligned}
V_{\varepsilon}(t) x-U_{\varepsilon}(t) x & =\left.U_{\varepsilon}(t, s) V_{\varepsilon}(s) x\right|_{s=0} ^{s=t}=\int_{0}^{t} U_{\varepsilon}(t, s)\left[P^{\prime}(s), P(s)\right] V_{\varepsilon}(s) x d s \\
& =\int_{0}^{t} U_{\varepsilon}(t, s)(B(s) A(s)-A(s) B(s)) V_{\varepsilon}(s) x d s
\end{aligned}
$$

for all $t \in I$. Since for every $t \in I$ the maps $\left.s \mapsto V_{\varepsilon}(s)\right|_{Y}$ and $\left.s \mapsto U_{\varepsilon}(t, s)\right|_{Y}$ are continuously differentiable on $[0, t]$ w.r.t. SOT of $L(Y, X)$ (Lemma 2.8) and hence belong to $W_{*}^{1, \infty}([0, t], L(Y, X))$, and since $s \mapsto B(s)$ belongs to $W_{*}^{1, \infty}([0, t], L(X, Y))$, we can further conclude, using Lemma 2.5, that $s \mapsto U_{\varepsilon}(t, s) B(s) V_{\varepsilon}(s) x$ is in $W^{1, \infty}([0, t], X)$, so 
that

$$
\begin{aligned}
& V_{\varepsilon}(t) x-U_{\varepsilon}(t) x=\varepsilon \int_{0}^{t} U_{\varepsilon}(t, s)\left(-\frac{1}{\varepsilon} A(s) B(s)+B(s) \frac{1}{\varepsilon} A(s)\right) V_{\varepsilon}(s) x d s \\
& \quad=\left.\varepsilon U_{\varepsilon}(t, s) B(s) V_{\varepsilon}(s) x\right|_{s=0} ^{s=t}-\varepsilon \int_{0}^{t} U_{\varepsilon}(t, s)\left(B^{\prime}(s)+B(s)\left[P^{\prime}(s), P(s)\right]\right) V_{\varepsilon}(s) x d s
\end{aligned}
$$

for all $t \in I$ and $\varepsilon \in(0, \infty)$, where $B^{\prime}$ denotes an arbitrary $W_{*}^{1, \infty}$-derivative of $B$. And from this, the conclusion of the theorem is obvious.

\subsection{An adiabatic theorem with non-uniform spectral gap condition}

We continue by proving an adiabatic theorem with non-uniform spectral gap condition where $\sigma($.$) falls into \sigma(A().) \backslash \sigma($.$) at countably many points that, in turn, accumulate$ at only finitely many points. We do so by extending Kato's proof from [26] where finitely many eigenvalue crossings for skew self-adjoint $A(t)$ are treated.

Theorem 3.2. Suppose $A(t): D \subset X \rightarrow X$ for every $t \in I$ is a linear map such that Condition 2.10 is satisfied with $\omega=0$. Suppose further that $\sigma(t)$ for every $t \in I$ is a compact subset of $\sigma(A(t))$, that $\sigma($.$) at countably many points accumulating at only$ finitely many points falls into $\sigma(A().) \backslash \sigma($.$) , and that I \backslash N \ni t \mapsto \sigma(t)$ is continuous, where $N$ denotes the set of those points where $\sigma($.$) falls into \sigma(A().) \backslash \sigma($.$) . And finally,$ for every $t \in I \backslash N$, let $P(t)$ be the projection associated with $A(t)$ and $\sigma(t)$ and suppose that $I \backslash N \ni t \mapsto P(t)$ extends to a map (again denoted by $P$ ) in $W_{*}^{2, \infty}(I, L(X))$. Then

$$
\sup _{t \in I}\left\|U_{\varepsilon}(t)-V_{\varepsilon}(t)\right\| \longrightarrow 0 \quad(\varepsilon \searrow 0),
$$

where $V_{\varepsilon}$ is the evolution system for $\frac{1}{\varepsilon} A+\left[P^{\prime}, P\right]$.

Proof. We first prove the assertion in the case where $\sigma($.$) at only finitely many points$ $t_{1}, \ldots, t_{m}$ (ordered in an increasing way) falls into $\sigma(A().) \backslash \sigma($.$) . So let \eta>0$. We partition the interval $I$ as follows:

$$
I=I_{0 \delta} \cup J_{1 \delta} \cup I_{1 \delta} \cup \cdots \cup J_{m \delta} \cup I_{m \delta},
$$

where $J_{i \delta}$ for $i \in\{1, \ldots, m\}$ is a relatively open subinterval of $I$ containing $t_{i}$ of length less than $\delta$ (which will be chosen in a minute) and where $I_{0 \delta}, \ldots, I_{m \delta}$ are the closed subintervals of $I$ lying between the subintervals $J_{1 \delta}, \ldots, J_{m \delta}$. In the following, we set $t_{i \delta}^{-}:=\inf I_{i \delta}$ and $t_{i \delta}^{+}:=\sup I_{i \delta}$ for $i \in\{0, \ldots, m\}$, and we choose $c$ so large that $\|P(s)\|$, $\left\|P^{\prime}(s)\right\|$ and $\left\|\left[P^{\prime}(s), P(s)\right]\right\| \leq c$ for all $s \in I$. Since

$$
\begin{aligned}
\left\|V_{\varepsilon}\left(t, t_{i-1 \delta}^{+}\right) x-U_{\varepsilon}\left(t, t_{i-1 \delta}^{+}\right) x\right\| & =\left\|\int_{t_{i-1 \delta}^{+}}^{t} U_{\varepsilon}(t, s)\left[P^{\prime}(s), P(s)\right] V_{\varepsilon}\left(s, t_{i-1 \delta}^{+}\right) x d s\right\| \\
& \leq M c M e^{M c} \delta\|x\|
\end{aligned}
$$


for every $t \in J_{i \delta}, x \in D$ and $\varepsilon \in(0, \infty)$, we can achieve - by choosing $\delta$ small enough that

$$
\sup _{t \in J_{i \delta}}\left\|V_{\varepsilon}\left(t, t_{i-1 \delta}^{+}\right)-U_{\varepsilon}\left(t, t_{i-1 \delta}^{+}\right)\right\|<\frac{\eta}{\left(4 M^{2} e^{2 M c}\right)^{m}}
$$

for every $\varepsilon \in(0, \infty)$ and $i \in\{1, \ldots, m\}$. And since $\left.\sigma()\right|_{.I_{i \delta}}$ at no point falls into $\left.(\sigma(A().) \backslash \sigma())\right|_{.I_{i \delta}}$, we conclude from the above adiabatic theorem with uniform spectral gap condition (applied to the restricted data $\left.A\right|_{I_{i \delta}},\left.\sigma\right|_{I_{i \delta}},\left.P\right|_{I_{i \delta}}$ ) that there is an $\varepsilon_{\delta} \in$ $(0, \infty)$ such that

$$
\sup _{t \in I_{i \delta}}\left\|V_{\varepsilon}\left(t, t_{i \delta}^{-}\right)-U_{\varepsilon}\left(t, t_{i \delta}^{-}\right)\right\|<\frac{\eta}{\left(4 M^{2} e^{2 M c}\right)^{m}}
$$

for every $\varepsilon \in\left(0, \varepsilon_{\delta}\right)$ and $i \in\{0, \ldots, m\}$. Combining the estimates (3.3) and (3.4) and using the product property from the definition of evolution systems, we readily conclude for every $i \in\{1, \ldots, m\}$ that

$$
\left\|V_{\varepsilon}(t)-U_{\varepsilon}(t)\right\|<\frac{\eta}{\left(4 M^{2} e^{2 M c}\right)^{m-i}} \leq \eta
$$

for all $t \in I_{i-1 \delta} \cup J_{i \delta} \cup I_{i \delta}$ and $\varepsilon \in\left(0, \varepsilon_{\delta}\right)$, and the desired conclusion follows.

We now prove the assertion in the case where $\sigma($.$) at infinitely many points accumu-$ lating at only finitely many points $t_{1}, \ldots, t_{m}$ (ordered in an increasing way) falls into $\sigma(A().) \backslash \sigma($.$) . In order to do so, we partition I$ and choose $\delta$ as we did above. We then obtain the estimate (3.3) as above and the estimate (3.4) by realizing that $\left.\sigma()\right|_{.I_{i \delta}}$ at only finitely many points falls into $\left.(\sigma(A().) \backslash \sigma())\right|_{.I_{i \delta}}$ (so that the case just proved can be applied). And from these estimates the conclusion follows as above.

It should be noticed that the hypotheses of the above adiabatic theorem allow the spectral subsets $\sigma(t)$ to be non-isolated in $\sigma(A(t))$ for all points $t \in N$, that is (by our definition of spectral gaps in Section 2.1), they also allow for some situations without spectral gap. It should also be noticed that, in the situation of the above theorem, one has $P(t) A(t) \subset A(t) P(t)$ for every $t \in I$ (although a priori this is clear only for $t \in I \backslash N$ ), which follows by a continuity argument. (Indeed, if $t_{0} \in I$ then it can be approximated by a sequence $\left(t_{n}\right)$ in $I \backslash N$. Since $t \mapsto\left(A\left(t_{0}\right)-1\right)(A(t)-1)^{-1}$ is NOT-continuous (by the $W_{*}^{1, \infty}$-regularity of $t \mapsto A(t)$ and Lemma 2.5), we see that for any $x \in D$

$$
\begin{aligned}
A\left(t_{0}\right) P\left(t_{n}\right) x & =\left(A\left(t_{0}\right)-1\right)\left(A\left(t_{n}\right)-1\right)^{-1} P\left(t_{n}\right)\left(A\left(t_{n}\right)-1\right) x+P\left(t_{n}\right) x \\
& \longrightarrow P\left(t_{0}\right) A\left(t_{0}\right) x \quad(n \rightarrow \infty)
\end{aligned}
$$

and therefore $P\left(t_{0}\right) A\left(t_{0}\right) \subset A\left(t_{0}\right) P\left(t_{0}\right)$ by the closedness of $A\left(t_{0}\right)$.) In particular, the evolution $V_{T}$ appearing in the above theorem really is adiabatic w.r.t. to $P$ by Proposition 2.14, as it should be. 


\subsection{Some remarks and examples}

We begin with four remarks concerning the adiabatic theorems with uniform and nonuniform spectral gap condition alike.

1. In the special situation where $\sigma(t)=\{\lambda(t)\}$ and $\lambda(t)$ is a pole of the resolvent map $(.-A(t))^{-1}$ of order at most $m_{0} \in \mathbb{N}$ for all $t \in I$, the operators $B(t)$ - used in the proof of the adiabatic theorems with spectral gap condition above to solve the commutator equation (3.1) - can be brought to a form, namely (3.6), which points the way to the solution of an appropriate (approximate) commutator equation in the adiabatic theorems without spectral gap condition below. Since $P P^{\prime} P, \bar{P} P^{\prime} \bar{P}=0$ by (2.4) (where $\bar{P}:=1-P)$ and

$$
(z-A(t))^{-1} P(t)=\frac{1}{z-\lambda(t)}\left(1-\frac{A(t)-\lambda(t)}{z-\lambda(t)}\right)^{-1} P(t)=\sum_{k=0}^{m_{0}-1} \frac{(A(t)-\lambda(t))^{k} P(t)}{(z-\lambda(t))^{k+1}}
$$

for every $z \in \rho(A(t))$ by Theorem 5.8-A of [50], we see that

$$
\begin{aligned}
B(t)=\sum_{k=0}^{m_{0}-1} & \frac{1}{2 \pi i} \int_{\gamma_{t}} \frac{\bar{R}(t, z)}{(z-\lambda(t))^{k+1}} d z P^{\prime}(t)(A(t)-\lambda(t))^{k} P(t) \\
& +\sum_{k=0}^{m_{0}-1}(A(t)-\lambda(t))^{k} P(t) P^{\prime}(t) \frac{1}{2 \pi i} \int_{\gamma_{t}} \frac{\bar{R}(t, z)}{(z-\lambda(t))^{k+1}} d z,
\end{aligned}
$$

and since the reduced resolvent map $z \mapsto \bar{R}(t, z):=\left(z-\left.A(t)\right|_{\bar{P}(t) D(A(t))}\right)^{-1} \bar{P}(t)$ is holomorphic on $\rho(A(t)) \cup\{\lambda(t)\}$, we further see - using Cauchy's theorem - that

$$
\begin{aligned}
B(t)=\sum_{k=0}^{m_{0}-1} \bar{R}(t, \lambda(t))^{k+1} & P^{\prime}(t)(A(t)-\lambda(t))^{k} P(t) \\
& +\sum_{k=0}^{m_{0}-1}(\lambda(t)-A(t))^{k} P(t) P^{\prime}(t) \bar{R}(t, \lambda(t))^{k+1} .
\end{aligned}
$$

2. In the even more special situation where $\sigma(t)=\{\lambda(t)\} \subset i \mathbb{R}$ and $\lambda(t)$ is a pole of the resolvent map $(.-A(t))^{-1}$, the hypotheses of the above adiabatic theorem with uniform spectral gap condition become essentially - apart from regularity conditions - equivalent to the hypotheses of the respective adiabatic theorem (Theorem 9) of [8], and a similar equivalence holds true for the above adiabatic theorem with non-uniform spectral gap condition. Indeed, if $\sigma(t)$ for every $t \in I$ is a singleton consisting of a pole $\lambda(t)$ on the imaginary axis, then the order $m(t)$ of nilpotence of $\left.A(t)\right|_{P(t) D}-\lambda(t)$ must be equal to 1 , since otherwise the relation

$$
\delta(\lambda(t)+\delta-A(t))^{-1} P(t)=\sum_{k=0}^{m(t)-1} \frac{(A(t)-\lambda(t))^{k}}{\delta^{k}} P(t)
$$


would yield the contradiction that the right hand side of (3.7) explodes as $\delta \searrow 0$ whereas the left hand side of (3.7) remains bounded as $\delta \searrow 0$ (by virtue of the $(M, 0)$-stability of $A$ and by $\lambda(t) \in i \mathbb{R}$ ). And therefore (by Theorem 5.8-A of [50]) $P(t) X=\operatorname{ker}(A(t)-\lambda(t)$ ) and $(1-P(t)) X=\operatorname{rg}(A(t)-\lambda(t))$ as in [8].

3. It is obvious from the proof of Theorem 3.1 that the assumption that $\sigma($.$) at no$ point fall into $\sigma(A().) \backslash \sigma($.$) and that t \mapsto \sigma(t)$ be continuous can be replaced by the weaker - but also less convenient - requirement that for each $t_{0} \in I$ there be a non-trivial closed interval $J_{t_{0}} \subset I$ containing $t_{0}$ and a cycle $\gamma_{t_{0}}$ such that $\operatorname{rg} \gamma_{t_{0}} \subset \rho(A(t))$ and

$$
\mathrm{n}\left(\gamma_{t_{0}}, \sigma(t)\right)=1 \quad \text { and } \quad \mathrm{n}\left(\gamma_{t_{0}}, \sigma(A(t)) \backslash \sigma(t)\right)=0
$$

for all $t \in J_{t_{0}}$. It can be shown that this weaker requirement still entails the upper semicontinuity of $t \mapsto \sigma(t)$ and hence - by Proposition 2.3 - it still ensures that $\sigma($. ) at no point falls into $\sigma(A().) \backslash \sigma($.$) or, in other words, that the spectral gap is uniform.$ (See Corollary 5.4 of [46] for a proof.) Consequently, if one adds to the thus weakened hypotheses the requirement that $t \mapsto \sigma(t)$ be lower semicontinuous (which - by Proposition 5.6 of [46] - is fulfilled if, for instance, $\sigma(t)$ is finite for every $t \in I$, and $0 \in \sigma(t)$ for all $t \in I$ or $0 \notin \sigma(t)$ for all $t \in I)$, one arrives at an adiabatic theorem equivalent to the original one above (Theorem 3.1). Similar remarks hold for the case of non-uniform spectral gap (Theorem 3.2).

4. We finally remark that the above adiabatic theorems - along with the commutator equation method used in their proofs - can be extended to several subsets $\sigma_{1}(t), \ldots$, $\sigma_{r}(t)$ of $\sigma(A(t))$ : if $A, \sigma_{j}, P_{j}$ for every $j \in\{1, \ldots, r\}$ satisfy the hypotheses of the above adiabatic theorem with uniform or non-uniform spectral gap and if $\sigma_{j}($.$) and \sigma_{l}($.$) for$ all $j \neq l$ fall into each other at only countably many points accumulating at only finitely many points, then there exists an evolution system $V_{\varepsilon}$, namely that for $\frac{1}{\varepsilon} A+K$ with

$$
K(t):=\frac{1}{2} \sum_{j=1}^{r+1}\left[P_{j}^{\prime}(t), P_{j}(t)\right] \quad \text { and } \quad P_{r+1}(t):=1-P(t):=1-\sum_{j=1}^{r} P_{j}(t),
$$

which on the one hand is simultaneously adiabatic w.r.t. all the $P_{j}$ by [26] and on the other hand well approximates the evolution system $U_{\varepsilon}$ for $\frac{1}{\varepsilon} A$ in the sense that

$$
\sup _{t \in I}\left\|U_{\varepsilon}(t)-V_{\varepsilon}(t)\right\| \longrightarrow 0 \quad(\varepsilon \searrow 0) .
$$

In order to see this, one has only to observe that $B(t):=\frac{1}{2} \sum_{j=1}^{r+1} B_{j}(t)$ with

$$
\begin{gathered}
B_{j}:=\frac{1}{2 \pi i} \int_{\gamma_{j}}(z-A)^{-1} P_{j}^{\prime}(z-A)^{-1} d z \quad(j \in\{1, \ldots, r\}) \\
B_{r+1}:=\frac{1}{2 \pi i} \int_{\gamma}(z-A)^{-1} P^{\prime}(z-A)^{-1} d z
\end{gathered}
$$

with $\gamma:=\gamma_{1}+\cdots+\gamma_{r}\left(\gamma_{j}=\gamma_{j t}\right.$ as in the proofs above $)$ and $P:=P_{1}+\cdots+P_{r}$ 
solves the commutator equation $B(t) A(t)-A(t) B(t) \subset K(t)$ for all points $t$ where no crossing takes place (because $\left[P_{r+1}^{\prime}, P_{r+1}\right]=\left[P^{\prime}, P\right]$ ) and then to proceed as in the proofs of the adiabatic theorems above. See also [14]. In the special case of skew self-adjoint operators $A(t)$ one can further refine the statement above: it is then possible to show by further adapting the commutator equation method - that even the evolution system $\bar{V}_{\varepsilon}$ for $\frac{1}{\varepsilon} A+\bar{K}$ with

$$
\bar{K}(t):=\frac{1}{2}\left(\left[\left(P_{r+1}^{-}\right)^{\prime}(t), P_{r+1}^{-}(t)\right]+\sum_{j=1}^{r}\left[P_{j}^{\prime}(t), P_{j}(t)\right]+\left[\left(P_{r+1}^{+}\right)^{\prime}(t), P_{r+1}^{+}(t)\right]\right)
$$

well approximates the evolution system $U_{\varepsilon}$ for $\frac{1}{\varepsilon} A$ - notice that $\bar{V}_{\varepsilon}$ is is not only adiabatic w.r.t. $P_{r+1}=P_{r+1}^{-}+P_{r+1}^{+}$but also w.r.t. $P_{r+1}^{-}$and $P_{r+1}^{+}$separately, where $P_{r+1}^{ \pm}(t)$ are the spectral projections of $A(t)$ corresponding to the parts $\sigma^{ \pm}(t)$ of the spectrum which on $i \mathbb{R}$ are located below resp. above all the compact parts $\sigma_{1}(t), \ldots, \sigma_{r}(t)$. In order to see this, set

$$
B_{r+1 n}^{ \pm}(t):=\frac{1}{2 \pi i} \int_{\gamma_{n t}^{ \pm}}(z-A(t))^{-1}\left(P_{r+1}^{ \pm}\right)^{\prime}(t)(z-A(t))^{-1} d z
$$

where $\gamma_{n t}^{ \pm}(\tau):= \pm \tau+c^{ \pm}(t)$ for $\tau \in[-n, n]$ with points $c^{ \pm}(t) \in i \mathbb{R}$ lying in the gap between $\sigma^{ \pm}(t)$ and the rest of $\sigma(A(t))$ and depending continuously differentiably on $t$, and observe that (by the skew self-adjointness of $A(t)$ )

$$
\begin{gathered}
P_{r+1 n}^{ \pm}(t) x:=\frac{1}{2 \pi i} \int_{\gamma_{n t}^{ \pm}}(z-A(t))^{-1} x d z \longrightarrow P_{r+1}^{ \pm}(t) x-\frac{1}{2} x \quad(n \rightarrow \infty) \\
\left\|B_{r+1 n}(t)\right\|,\left\|B_{r+1 n}^{\prime}(t)\right\| \leq \int_{-\infty}^{\infty} \frac{\text { and }}{\operatorname{dist}\left(\gamma_{n t}^{ \pm}(\tau), \sigma(A(t))\right)^{2}} d \tau \leq C<\infty \quad(n \in \mathbb{N}, t \in I) .
\end{gathered}
$$

A slightly less general general statement was first proven in [38] by a different method than the commutator equation technique indicated above.

We now move on to discuss some examples. In the first - very simple - example, $t \mapsto A(t)$ is only $W_{*}^{1, \infty}$-regular and only $(M, 0)$-stable (without being SOT-continuously differentiable or $(1,0)$-stable), which means that this example lies outside the scope of the previously known adiabatic theorems.

Example 3.3. Suppose $A, \sigma, P$ with $A(t)=R(t)^{-1} A_{0}(t) R(t), \sigma(t)=\{\lambda(t)\}, P(t)=$ $R(t)^{-1} P_{0} R(t)$, and $R(t)=e^{C t}$ are given as follows in $X:=\ell^{p}\left(I_{2}\right) \times \ell^{p}\left(I_{1}\right)$ (where $p \in[1, \infty)):$

$$
A_{0}(t):=\left(\begin{array}{cc}
\lambda(t)+\alpha(t) N & 0 \\
0 & \mu(t)
\end{array}\right) \quad \text { and } \quad P_{0}:=\left(\begin{array}{ll}
1 & 0 \\
0 & 0
\end{array}\right),
$$

where $\lambda, \alpha, N$ satisfy Condition 2.16 and where $\mu(t) \in\{\operatorname{Re} z \leq 0\}$ is such that $\lambda($. falls into $\mu($.$) at only countably many points accumulating at only finitely many points.$ 
Additionally, choose the family $\lambda+\alpha N$ to be not $(1,0)$-stable (which, by the discussion after Lemma 2.17, can easily be achieved), the functions $t \mapsto \lambda(t), \alpha(t), \mu(t)$ to be Lipschitz continuous without being continuously differentiable, and

$$
C:=\left(\begin{array}{ccc}
0 & 0 & 0 \\
0 & 0 & 1 \\
0 & -1 & 0
\end{array}\right) .
$$

Since $A_{0}$ by Lemma 2.17 is $\left(M_{0}, 0\right)$-stable for some $M_{0} \in[1, \infty)$, the twisted family $A$ is $(M, 0)$-stable for another $M \in[1, \infty)$ by Lemma 2.9. And since $P_{0}$ is obviously associated with $A_{0}(t)$ and $\sigma(t)$ for every $t \in I \backslash N$ (where $N$ denotes the set of those points in $I$ where $\lambda($.$) falls into \mu()$.$) , the same is true for P(t)$ and $A(t)$ instead of $P_{0}, A_{0}(t)$. So the hypotheses of the adiabatic theorem with non-uniform spectral gap condition (Theorem 3.2) are fulfilled and therefore

$$
(1-P(t)) U_{\varepsilon}(t) P(0) \longrightarrow 0 \quad(\varepsilon \searrow 0)
$$

uniformly in $t \in I$, but this does not already follow from the trivial adiabatic theorems above (Proposition 2.15 (i) and (ii)). Indeed, as $\left[P_{0}, C\right] \neq 0$, it follows that $P^{\prime}(t) \neq 0$ for every $t \in I$. And if $\mu$ is chosen in such a way that $\mu\left(t_{0}\right)=0$ for some $t_{0} \in[0,1)$, then it follows that the (block diagonal!) evolution $U_{0 \varepsilon}$ for $\frac{1}{\varepsilon} A_{0}$ for no $\gamma>0$ satisfies the estimate (2.5) uniformly in $\varepsilon \in(0, \infty)$, whence by Proposition 2.13 the same is true for the evolution $\tilde{U}_{0 \varepsilon}$ for $\frac{1}{\varepsilon} A_{0}+C=\frac{1}{\varepsilon} A_{0}+R^{\prime} R^{-1}$ and finally (by the proof of Corollary 2.12) also for the evolution $U_{\varepsilon}$ for $\frac{1}{\varepsilon} A$ that we are interested in.

In the next exmaple, the spectral subsets $\sigma(t)=\{\lambda(t)\}$ are singletons consisting of spectral values $\lambda(t) \in i \mathbb{R}$ of $A(t)$ that are not eigenvalues and, a fortiori, are not poles (but essential singularities) of $(.-A(t))^{-1}$. In particular, the adiabatic theorem with spectral gap condition from [8] cannot be applied here (also see Example 4 of [8]). We make use of the Volterra operator $V$ in $L^{2}([0,1])$ defined by

$$
(V f)(t):=\int_{0}^{t} f(\tau) d \tau \quad(t \in I)
$$

Since $V$ is quasinilpotent and both $V$ and $V^{*}$ are injective, it follows that $\sigma(V)=\{0\}=$ $\sigma_{c}(V)$.

Example 3.4. Suppose $A, \sigma, P$ with $A(t)=R(t)^{-1} A_{0}(t) R(t), \sigma(t)=\{\lambda(t)\}:=\{0\}$, $P(t)=R(t)^{-1} P_{0} R(t)$, and $R(t)=e^{C t}$ are given as follows in $X:=L^{2}(I) \times L^{2}(I)$ :

$$
A_{0}(t):=\left(\begin{array}{cc}
-V & 0 \\
0 & a(t)+M_{f}
\end{array}\right) \text { and } P_{0}:=\left(\begin{array}{ll}
1 & 0 \\
0 & 0
\end{array}\right)
$$

where $V$ is the Volterra operator defined above and where $f: I \rightarrow \mathbb{C}$ is a measurable function with ess-rg $f=[-1,0]$. Additionally, suppose the function $t \mapsto a(t) \in(-\infty, 0]$ 
is Lipschitz continuous and falls into 0 at only countably many points accumulating at only finitely many points, and

$$
C:=\left(\begin{array}{cc}
0 & 1 \\
-1 & 0
\end{array}\right)
$$

Since $-V$ and $a(t)+M_{f}$ are dissipative in $L^{2}(I)$, the family $A_{0}$ is $(1,0)$-stable and, by the unitarity of the rotation operators $e^{C t}=R(t)$, the same goes for $A$. Also, since $P_{0}$ commutes with $A_{0}(t)$ and

$$
\begin{gathered}
\sigma\left(\left.A_{0}(t)\right|_{P_{0} X}\right)=\sigma(-V)=\{0\}=\sigma(t), \\
\sigma\left(\left.A_{0}(t)\right|_{\left(1-P_{0}\right) X}\right)=\sigma\left(a(t)+M_{f}\right)=a(t)+[-1,0]=\sigma\left(A_{0}(t)\right) \backslash \sigma(t)
\end{gathered}
$$

for every $t \in I \backslash N, P_{0}$ is associated with $A_{0}(t)$ and $\sigma(t)$ for every $t \in I \backslash N$ and hence the same holds true for $P(t)$ and $A(t)$ instead of $P_{0}, A_{0}(t)$. All other hypotheses of Theorem 3.2 are clear.

We finally give a simple example showing that the conclusion of the above adiabatic theorems will, in general, fail if the evolution systems $U_{\varepsilon}$ for $\frac{1}{\varepsilon} A$ are not bounded in $\varepsilon$ (and hence $A$ is not $(M, 0)$-stable).

Example 3.5. Suppose $A, \sigma, P$ with $A(t):=R(t)^{-1} A_{0}(t) R(t), \sigma(t):=\{\lambda(t)\}$ and $P(t):=R(t)^{-1} P_{0} R(t)$ are given as follows in $X:=\ell^{2}\left(I_{2}\right)$ :

$$
A_{0}(t):=\left(\begin{array}{cc}
\lambda(t) & 0 \\
0 & 0
\end{array}\right), \quad P_{0}:=\left(\begin{array}{ll}
1 & 0 \\
0 & 1
\end{array}\right), \quad R(t):=e^{C t} \quad \text { with } \quad C:=2 \pi\left(\begin{array}{cc}
0 & 1 \\
-1 & 0
\end{array}\right),
$$

and $t \mapsto \lambda(t) \in[0, \infty)$ is Lipschitz continuous such that $\lambda($.$) at only countably many$ points accumulating at only finitely many points falls into 0 . Then all the hypotheses of Theorem 3.2 are fullfilled with the sole exception that $A$ is not $(M, 0)$-stable (because $\sigma(A(t))=\{0, \lambda(t)\}$ is contained in the closed left half-plane only for countably many $t \in I)$ and, in fact, the conclusion of this theorems fails. Indeed, since

$$
R(t)=e^{C t}=\left(\begin{array}{cc}
\cos (2 \pi t) & \sin (2 \pi t) \\
-\sin (2 \pi t) & \cos (2 \pi t)
\end{array}\right)
$$

we see that

$$
A(t)=R(t)^{-1} A_{0}(t) R(t)=\lambda(t)\left(\begin{array}{cc}
\cos ^{2}(2 \pi t) & \cos (2 \pi t) \sin (2 \pi t) \\
\cos (2 \pi t) \sin (2 \pi t) & \sin ^{2}(2 \pi t)
\end{array}\right)
$$

is a positive linear map (in the lattice sense) for all $t \in\left[0, t_{0}\right]$ with $t_{0}:=\frac{1}{4}$. And since $1-P\left(t_{0}\right)=P_{0}$, we see (by the series expansion for $U_{\varepsilon}$ ) that

$$
\begin{aligned}
& \left\|\left(1-P\left(t_{0}\right)\right) U_{\varepsilon}\left(t_{0}\right) P(0) e_{1}\right\|=\left|\left\langle e_{1}, U_{\varepsilon}\left(t_{0}\right) e_{1}\right\rangle\right|=\left\langle e_{1}, U_{\varepsilon}\left(t_{0}\right) e_{1}\right\rangle \\
& \geq 1+\frac{1}{\varepsilon} \int_{0}^{t_{0}}\left\langle e_{1}, A(\tau) e_{1}\right\rangle d \tau=1+\frac{1}{\varepsilon} \int_{0}^{t_{0}} \lambda(\tau) \cos ^{2}(2 \pi \tau) d \tau,
\end{aligned}
$$

which right hand side does not converge to 0 as $\varepsilon \searrow 0$, as desired. 
An example with non-diagonalizable $A(t)$ and $\sigma(A(t))=\{0, i\}$ showing as well that the conclusion of the above adiabatic theorems will generally fail if the family $A$ is not $(M, 0)$-stable can be found in Joye's paper [25] at the end of Section 1. A generic version of this (non-generic) example is given by the following data: $A(t):=R(t)^{-1} A_{0}(t) R(t)$, $\lambda(t):=0, P(t):=R(t)^{-1} P_{0} R(t)$ in $X:=\ell^{2}\left(I_{3}\right)$, where

$$
A_{0}(t)=A_{0}:=\left(\begin{array}{lll}
0 & i & 0 \\
0 & 0 & 0 \\
0 & 0 & i
\end{array}\right) \quad \text { and } \quad R(t):=e^{C t} \quad \text { with } \quad C:=\left(\begin{array}{ccc}
0 & 0 & 0 \\
i k & 0 & k \\
1 & -1 & 0
\end{array}\right)
$$

for a parameter $k \in(-\infty, 0)$ and where $P_{0}$ is the orthogonal projection onto $\operatorname{span}\left\{e_{1}, e_{2}\right\}$.

\section{Adiabatic theorems without spectral gap condition for time-independent domains}

After having established general adiabatic theorems with spectral gap condition in Section 3, we can now prove an adiabatic theorem without spectral gap condition for general operators $A(t)$ with not necessarily weakly semisimple spectral values $\lambda(t)$ : in Section 4.1 it appears in a qualitative version and in Section 4.2 in a quantitatively refined version, which, in turn, is applied to the special case of spectral operators of scalar type. We thereby generalize the recent adiabatic theorems without spectral gap condition of Avron, Fraas, Graf, Grech from [8] and of Schmid from [46], which theorems - although independently obtained - are essentially the same (save for some regularity subtleties). In these theorems - which so far are the only ones to cover not necessarily skew self-adjoint operators $A(t)$ in the case without spectral gap - the considered eigenvalues $\lambda(t)$ are required to be weakly semisimple. Since, however, the eigenvalues of general operators are generally not weakly semisimple (Section 4.3 provides simple examples for this), it is natural to ask whether one can do without the requirement of weak semisimplicity (or, in other words, weak 1-associatedness). And the theorems below show that one actually can: indeed, in essence, it suffices to require just weak associatedness - which, at the beginning of Section 2.1, has been explained to be a fairly natural assumption.

\subsection{A qualitative adiabatic theorem without spectral gap condition}

We begin with two lemmas that will be crucial in the proofs of the presented adiabatic theorems without spectral gap condition.

Lemma 4.1. Suppose that $A: D(A) \subset X \rightarrow X$ is a densely defined closed linear map and that $\lambda \in \sigma(A)$ and $\delta_{0} \in(0, \infty)$ and $\vartheta_{0} \in \mathbb{R}$ such that $\lambda+\delta e^{i \vartheta_{0}} \in \rho(A)$ for all $\delta \in\left(0, \delta_{0}\right]$. Suppose finally that $P$ is a bounded projection in $X$ such that $P A \subset A P$ and

$$
(1-P) X \subset \overline{\operatorname{rg}(A-\lambda)^{m_{0}}}
$$

for some $m_{0} \in \mathbb{N}$, and that there is $M_{0} \in(0, \infty)$ such that

$$
\left\|\left(\lambda+\delta e^{i \vartheta_{0}}-A\right)^{-1}(1-P)\right\| \leq \frac{M_{0}}{\delta}
$$


for all $\delta \in\left(0, \delta_{0}\right]$. Then $\delta\left(\lambda+\delta e^{i \vartheta_{0}}-A\right)^{-1}(1-P) x \longrightarrow 0$ as $\delta \searrow 0$ for all $x \in X$.

Proof. If $x \in \operatorname{rg}(A-\lambda)^{m_{0}}$, then $x=(\lambda-A)^{m_{0}} x_{0}$ for some $x_{0} \in D\left(A^{m_{0}}\right)$ and, by the assumed resolvent estimate,

$$
\begin{aligned}
\delta\left(\lambda+\delta e^{i \vartheta_{0}}-A\right)^{-1} \bar{P} x= & \delta\left(\lambda+\delta e^{i \vartheta_{0}}-A\right)^{-1} \bar{P}\left(-\delta e^{i \vartheta_{0}}\right)^{m_{0}} x_{0} \\
& +\delta \sum_{k=1}^{m_{0}}\left(\begin{array}{c}
m_{0} \\
k
\end{array}\right)\left(\lambda+\delta e^{i \vartheta_{0}}-A\right)^{k-1}\left(-\delta e^{i \vartheta_{0}}\right)^{m_{0}-k} \bar{P} x_{0} \longrightarrow 0
\end{aligned}
$$

as $\delta \searrow 0$, where of course $\bar{P}:=1-P$. And if $x \in X$, then $\bar{x}:=\bar{P} x$ can be approximated arbitrarily well by elements $y$ of $\operatorname{rg}(A-\lambda)^{m_{0}}$ and therefore

$$
\delta\left(\lambda+\delta e^{i \vartheta_{0}}-A\right)^{-1} \bar{P} x=\delta\left(\lambda+\delta e^{i \vartheta_{0}}-A\right)^{-1} \bar{P}(\bar{x}-y)+\delta\left(\lambda+\delta e^{i \vartheta_{0}}-A\right)^{-1} \bar{P} y
$$

can be made arbitrarily small for $\delta$ small enough by the assumed resolvent estimate and by what has just been shown.

Lemma 4.2. Suppose that $A(t): D(A(t)) \subset X \rightarrow X$ for every $t \in I$ is a densely defined closed linear map, that $\lambda(t) \in \sigma(A(t))$ and $\delta_{0} \in(0, \infty)$ and $\vartheta(t) \in \mathbb{R}$ such that $\lambda(t)+\delta e^{i \vartheta(t)} \in \rho(A(t))$ for all $\delta \in\left(0, \delta_{0}\right]$ and $t \in I$ and such that

$$
t \mapsto\left(\lambda(t)+\delta e^{i \vartheta(t)}-A(t)\right)^{-1}
$$

is in $W_{*}^{1, \infty}(I, L(X))$ (resp. SOT-continuously differentiable) and $t \mapsto \lambda(t)$ as well as $t \mapsto e^{i \vartheta(t)}$ is Lipschitz continuous (resp. continuously differentiable). Suppose further that $P(t)$ for every $t \in I$ is a bounded projection in $X$ such that $P(t) A(t) \subset A(t) P(t)$ for every $t \in I$ and $P(t) X \subset \operatorname{ker}(A(t)-\lambda(t))^{m_{0}}$ for every $t \in I$ (where $m_{0} \in \mathbb{N}$ ) and $t \mapsto P(t)$ is SOT-continuously differentiable. Then

$$
t \mapsto(A(t)-\lambda(t)) P(t)
$$

is in $W_{*}^{1, \infty}(I, L(X))$ (resp. SOT-continuously differentiable) and, in particular, for every $\varepsilon \in(0, \infty)$ the evolution system $V_{0 \varepsilon}$ for $\frac{1}{\varepsilon} A P+\left[P^{\prime}, P\right]$ exists on $X$ and is adiabatic w.r.t. $P$. If, in addition, the evolution system $U_{\varepsilon}$ for $\frac{1}{\varepsilon} A$ exists on $D(A(t))$ and if there is an $M \in(0, \infty)$ such that $\left\|U_{\varepsilon}(t, s)\right\| \leq M$ for all $(s, t) \in \Delta$ and $\varepsilon \in(0, \infty)$, then

$$
\left\|V_{0 \varepsilon}(t, s) P(s)\right\| \leq M c e^{M c(t-s)}
$$

for all $(s, t) \in \Delta$, where $c$ is an upper bound of $t \mapsto\|P(t)\|,\left\|P^{\prime}(t)\right\|$.

Proof. Since $P(t) X \subset \operatorname{ker}(A(t)-\lambda(t))^{m_{0}} \subset D\left(A(t)^{m_{0}}\right)$ for every $t \in I$, we see that $(A(t)-\lambda(t)) P(t)$ is a bounded linear map in $X$ for every $t \in I$ and that

$$
\begin{gathered}
t \mapsto(A(t)-\lambda(t)) P(t)=(A(t)-\lambda(t)) S_{\delta}(t) S_{\delta}(t)^{m_{0}-1}\left(A(t)-\lambda(t)-\delta e^{i \vartheta(t)}\right)^{m_{0}} P(t) \\
=\left(1+\delta e^{i \vartheta(t)} S_{\delta}(t)\right) \sum_{k=0}^{m_{0}-1}\left(\begin{array}{c}
m_{0} \\
k
\end{array}\right)\left(-\delta e^{i \vartheta(t)}\right)^{m_{0}-k} . \\
\cdot S_{\delta}(t)^{m_{0}-1-k}\left(1+\delta e^{i \vartheta(t)} S_{\delta}(t)\right)^{k} P(t)
\end{gathered}
$$


is in $W_{*}^{1, \infty}(I, L(X))$ (resp. SOT-continuously differentiable) by Lemma 2.5, because

$$
t \mapsto S_{\delta}(t):=\left(A(t)-\lambda(t)-\delta e^{i \vartheta(t)}\right)^{-1}
$$

is of that regularity by Lemma 2.5. In particular, $t \mapsto A(t) P(t)$ is SOT-continuous and therefore the evolution system $V_{\varepsilon}$ for $\frac{1}{\varepsilon} A P+\left[P^{\prime}, P\right]$ exists on $X$ and (by virtue of Proposition 2.14) is adiabatic w.r.t. $P$ for every $\varepsilon \in(0, \infty)$. Suppose finally that the additional assumption concerning the evolution system $U_{\varepsilon}$ for $\frac{1}{\varepsilon} A$ is satisfied. Since for all $x \in X$ and $(s, t) \in \Delta$ the map $[s, t] \ni \tau \mapsto U_{\varepsilon}(t, \tau) V_{0 \varepsilon}(\tau, s) P(s) x$ is continuous and right differentiable (Lemma 2.6) with bounded (even continuous) right derivative

$$
\begin{aligned}
\tau \mapsto & U_{\varepsilon}(t, \tau)\left(\frac{1}{\varepsilon} A(\tau) P(\tau)-\frac{1}{\varepsilon} A(\tau)+\left[P^{\prime}(\tau), P(\tau)\right]\right) V_{0 \varepsilon}(\tau, s) P(s) x \\
& =U_{\varepsilon}(t, \tau) P^{\prime}(\tau) V_{0 \varepsilon}(\tau, s) P(s) x
\end{aligned}
$$

(where in the last equality the adiabaticity of $V_{\varepsilon}$ w.r.t. $P$ and (2.4) have been used), it follows from Lemma 2.7 that

$$
\begin{aligned}
V_{0 \varepsilon}(t, s) P(s) x-U_{\varepsilon}(t, s) P(s) x & =\left.U_{\varepsilon}(t, \tau) V_{0 \varepsilon}(\tau, s) P(s) x\right|_{\tau=s} ^{\tau=t} \\
& =\int_{s}^{t} U_{\varepsilon}(t, \tau) P^{\prime}(\tau) V_{0 \varepsilon}(\tau, s) P(s) x d \tau
\end{aligned}
$$

for all $(s, t) \in \Delta$ and $x \in X$. And this integral equation, by the Gronwall inequality, yields the desired estimate for $V_{0 \varepsilon}(t, s) P(s)$.

With these lemmas at hand, we can now prove the announced general adiabatic theorem without spectral gap condition for not necessarily weakly semisimple eigenvalues. Similarly to the works [7] of Avron and Elgart and [52] of Teufel its proof rests upon solving a suitable approximate commutator equation. In this undertaking the insights gained in Section 3, especially formula (3.6), will prove indispensable. (Alternatively, part (i) of the theorem could also - less elegantly - be based upon a suitable iterated partial integration argument, but part (ii) could not.)

Theorem 4.3. Suppose $A(t): D \subset X \rightarrow X$ for every $t \in I$ is a linear map such that Condition 2.10 is satisfied with $\omega=0$. Suppose further that $\lambda(t)$ for every $t \in I$ is an eigenvalue of $A(t)$, and that there are numbers $\delta_{0} \in(0, \infty)$ and $\vartheta(t) \in \mathbb{R}$ such that $\lambda(t)+\delta e^{i \vartheta(t)} \in \rho(A(t))$ for all $\delta \in\left(0, \delta_{0}\right]$ and $t \in I$ and such that $t \mapsto \lambda(t)$ and $t \mapsto e^{i \vartheta(t)}$ are Lipschitz continuous. Suppose finally that $P(t)$ for every $t \in I$ is a bounded projection in $X$ commuting with $A(t)$ such that $P(t)$ for almost every $t \in I$ is weakly associated with $A(t)$ and $\lambda(t)$, suppose there is an $M_{0} \in(0, \infty)$ such that

$$
\left\|\left(\lambda(t)+\delta e^{i \vartheta(t)}-A(t)\right)^{-1}(1-P(t))\right\| \leq \frac{M_{0}}{\delta}
$$

for all $\delta \in\left(0, \delta_{0}\right]$ and $t \in I$, let $\operatorname{rk} P(0)<\infty$ and suppose that $t \mapsto P(t)$ is SOTcontinuously differentiable. 
(i) If $X$ is arbitrary (not necessarily reflexive), then

$$
\sup _{t \in I}\left\|\left(U_{\varepsilon}(t)-V_{0 \varepsilon}(t)\right) P(0)\right\| \longrightarrow 0 \quad(\varepsilon \searrow 0),
$$

where $V_{0 \varepsilon}$ is the evolution system for $\frac{1}{\varepsilon} A P+\left[P^{\prime}, P\right]$ on $X$ for every $\varepsilon \in(0, \infty)$.

(ii) If $X$ is reflexive and $t \mapsto P(t)$ is norm continuously differentiable, then

$$
\sup _{t \in I}\left\|U_{\varepsilon}(t)-V_{\varepsilon}(t)\right\| \longrightarrow 0 \quad(\varepsilon \searrow 0)
$$

whenever the evolution system $V_{\varepsilon}$ for $\frac{1}{\varepsilon} A+\left[P^{\prime}, P\right]$ exists on $D$ for every $\varepsilon \in(0, \infty)$.

Proof. We begin with some preparations which will be used in the proof of both assertion (i) and assertion (ii). As a first preparatory step, we show that $t \mapsto P(t)$ is in $W_{*}^{1, \infty}(I, L(X, Y))$ and that there is an $m_{0} \in \mathbb{N}$ such that $P(t) X \subset \operatorname{ker}(A(t)-\lambda(t))^{m_{0}}$ for every $t \in I$. Since $P(t)$ for almost every $t \in I$ is weakly associated with $A(t)$ and $\lambda(t)$ and since

$$
\operatorname{dim} P(t) X=\operatorname{rk} P(0) X<\infty
$$

for every $t \in I$ (which equality is due to the continuity of $t \mapsto P(t)$ and Lemma VII.6.7 of [18]), there is a $t$-independent constant $m_{0} \in \mathbb{N}$ - for instance, $m_{0}:=\operatorname{rk} P(0)-$ such that $P(t)$ is weakly $m_{0}$-associated with $A(t)$ and $\lambda(t)$ for almost every $t \in I$. In particular, it follows from Theorem 2.1 that

$$
P(t) X \subset \operatorname{ker}(A(t)-\lambda(t))^{m_{0}} \quad \text { and } \quad(1-P(t)) X \subset \overline{\operatorname{rg}(A(t)-\lambda(t))^{m_{0}}}
$$

for almost every $t \in I$. We now show that the inclusion

$$
P(t) X \subset \operatorname{ker}(A(t)-\lambda(t))^{m_{0}}
$$

actually holds for every $t \in I$, in order to be able to apply Lemma 4.2. Since $P(t)$ commutes with $A(t)$ and $\operatorname{dim} P(t) X=\operatorname{rk} P(0)<\infty$ for every $t \in I$, one has $P(t) D(A(t))=$ $P(t) X$ and hence $P(t) X \subset D\left(A(t)^{m_{0}}\right)$ as well as

$$
(A(t)-\lambda(t))^{m_{0}} P(t)=((A(t)-\lambda(t)) P(t))^{m_{0}}
$$

for every $t \in I$. So in order to establish (4.3) it suffices to show that $t \mapsto A(t) P(t)$ is SOT-continuous, because the set $I \backslash N$ of those $t \in I$ where $P(t)$ is weakly associated with $A(t)$ and $\lambda(t)$ is dense in $I$. Analagously to (4.1) it follows that

$$
\begin{aligned}
I \backslash N \ni t \mapsto A(0) P(t)=A(0) S_{\delta}(t) \sum_{k=0}^{m_{0}-1}\left(\begin{array}{c}
m_{0} \\
k
\end{array}\right)\left(-\delta e^{i \vartheta(t)}\right)^{m_{0}-k} . \\
\cdot S_{\delta}(t)^{m_{0}-1-k}\left(1+\delta e^{i \vartheta(t)} S_{\delta}(t)\right)^{k} P(t)
\end{aligned}
$$


extends to a $W_{*}^{1, \infty}(I, L(X))$-regular map and the closedness of $A(0)$ implies that the $W_{*}^{1, \infty}(I, L(X))$-regular extension is given by $I \ni t \mapsto A(0) P(t)$. In other words, $t \mapsto P(t)$ (by the definition of the norm of $Y$ in Condition 2.10) belongs to $W_{*}^{1, \infty}(I, L(X, Y))$. In particular, $t \mapsto A(t) P(t)$ is SOT-continuous and (4.3) follows.

As a second preparatory step, we solve - in accordance with the proof of the adiabatic theorems with spectral gap condition - a suitable (approximate) commutator equation. Inspired by (3.6), we define the operators

$$
\begin{aligned}
B_{n \boldsymbol{\delta}}(t):=\sum_{k=0}^{m_{0}-1}\left(\prod_{i=1}^{k+1} \bar{R}_{\delta_{i}}(t)\right) Q_{n}(t)(\lambda(t)-A(t))^{k} P(t) \\
\quad+\sum_{k=0}^{m_{0}-1}(\lambda(t)-A(t))^{k} P(t) Q_{n}(t)\left(\prod_{i=1}^{k+1} \bar{R}_{\delta_{i}}(t)\right)
\end{aligned}
$$

for $n \in \mathbb{N}, \boldsymbol{\delta}:=\left(\delta_{1}, \ldots, \delta_{m_{0}}\right) \in\left(0, \delta_{0}\right]^{m_{0}}$ and $t \in I$, where

$$
\bar{R}_{\delta}(t):=R_{\delta}(t) \bar{P}(t) \quad \text { with } \quad R_{\delta}(t):=\left(\lambda(t)+\delta e^{i \vartheta(t)}-A(t)\right)^{-1} \text { and } \bar{P}(t):=1-P(t)
$$

for $\delta \in\left(0, \delta_{0}\right]$, and where

$$
Q_{n}(t):=\int_{0}^{1} j_{\frac{1}{n}}(t-r) P^{\prime}(r) d r .
$$

In other words, $Q_{n}$ is obtained from $P^{\prime}$ by mollification, whence $t \mapsto Q_{n}(t)$ is SOTcontinuously differentiable and $Q_{n}(t) \longrightarrow P^{\prime}(t)$ as $n \rightarrow \infty$ w.r.t. SOT for $t \in(0,1)$ and

$$
\sup \left\{\left\|Q_{n}(t)\right\|: t \in I, n \in \mathbb{N}\right\} \leq \sup _{t \in I}\left\|P^{\prime}(t)\right\| .
$$

We now show that the operators $B_{n} \boldsymbol{\delta}(t)$ satisfy the approximate commutator equation

$$
B_{n \delta}(t) A(t)-A(t) B_{n \delta}(t)+C_{n \delta}(t) \subset\left[Q_{n}(t), P(t)\right]
$$

with remainder terms $C_{n} \boldsymbol{\delta}(t)$ that will have to be suitably controlled below. Since

$$
(\lambda-A)\left(\prod_{i=1}^{k+1} \bar{R}_{\delta_{i}}\right)=\left(\prod_{1 \leq i \leq k} \bar{R}_{\delta_{i}}\right)-\delta_{k+1} e^{i \vartheta}\left(\prod_{i=1}^{k+1} \bar{R}_{\delta_{i}}\right) \supset\left(\prod_{i=1}^{k+1} \bar{R}_{\delta_{i}}\right)(\lambda-A)
$$

(the $t$-dependence being suppressed here and in the following lines for the sake of convenience), it follows that

$$
\begin{aligned}
& (\lambda-A) B_{n \boldsymbol{\delta}}=\sum_{k=0}^{m_{0}-1}\left(\prod_{1 \leq i \leq k} \bar{R}_{\delta_{i}}\right) Q_{n}(\lambda-A)^{k} P+\sum_{k=0}^{m_{0}-1}(\lambda-A)^{k+1} P Q_{n}\left(\prod_{i=1}^{k+1} \bar{R}_{\delta_{i}}\right)-C_{n \boldsymbol{\delta}}^{+} \\
& B_{n \boldsymbol{\delta}}(\lambda-A) \subset \sum_{k=0}^{m_{0}-1}\left(\prod_{i=1}^{k+1} \bar{R}_{\delta_{i}}\right) Q_{n}(\lambda-A)^{k+1} P+\sum_{k=0}^{m_{0}-1}(\lambda-A)^{k} P Q_{n}\left(\prod_{1 \leq i \leq k} \bar{R}_{\delta_{i}}\right)-C_{n \boldsymbol{\delta}}^{-}
\end{aligned}
$$


where we used the abbreviations

$$
\begin{aligned}
C_{n \boldsymbol{\delta}}^{+}:=\sum_{k=0}^{m_{0}-1} \delta_{k+1} e^{i \vartheta}\left(\prod_{i=1}^{k+1} \bar{R}_{\delta_{i}}\right) Q_{n}(\lambda-A)^{k} P, \\
C_{n \boldsymbol{\delta}}^{-}:=\sum_{k=0}^{m_{0}-1}(\lambda-A)^{k} P Q_{n} \delta_{k+1} e^{i \vartheta}\left(\prod_{i=1}^{k+1} \bar{R}_{\delta_{i}}\right) .
\end{aligned}
$$

Subtracting $B_{n} \delta(\lambda-A)$ from $(\lambda-A) B_{n} \delta$ and noticing that, by doing so, of all the summands not belonging to $C_{n \boldsymbol{\delta}}^{+}, C_{n \boldsymbol{\delta}}^{-}$only

$$
Q_{n} P-\left(\prod_{i=1}^{m_{0}} \bar{R}_{\delta_{i}}\right) Q_{n}(\lambda-A)^{m_{0}} P+(\lambda-A)^{m_{0}} P Q_{n}\left(\prod_{i=1}^{m_{0}} \bar{R}_{\delta_{i}}\right)-P Q_{n}=\left[Q_{n}, P\right]
$$

remains (remember (4.3)), we see that

$$
B_{n \boldsymbol{\delta}} A-A B_{n \boldsymbol{\delta}} \subset\left[Q_{n}, P\right]-C_{n \boldsymbol{\delta}}^{+}+C_{n \boldsymbol{\delta}}^{-}
$$

which is nothing but (4.5) if one defines $C_{n \boldsymbol{\delta}}:=C_{n \boldsymbol{\delta}}^{+}-C_{n \boldsymbol{\delta}}^{-}$.

As a third preparatory step we observe that $t \mapsto B_{n} \delta(t)$ belongs to $W_{*}^{1, \infty}(I, L(X, Y))$ and estimate $B_{n \boldsymbol{\delta}}$ as well as $B_{n \boldsymbol{\delta}}^{\prime}$. Since $t \mapsto A(t)-\lambda(t)-\delta e^{i \vartheta(t)}$ is in $W_{*}^{1, \infty}(I, L(Y, X))$,

$$
t \mapsto(A(t)-\lambda(t))^{k} P(t)=((A(t)-\lambda(t)) P(t))^{k}
$$

is in $W_{*}^{1, \infty}(I, L(X))$ by Lemma 4.2, and $t \mapsto P(t)$ is in $W_{*}^{1, \infty}(I, L(X, Y))$ by the first preparatory step, the asserted $W_{*}^{1, \infty}(I, L(X, Y))$-regularity of $t \mapsto B_{n} \delta(t)$ follows from Lemma 2.5. Additionally, there is a constant $c$ such that

$$
\sup _{t \in I}\left\|B_{n} \boldsymbol{\delta}(t)\right\| \leq \sum_{k=1}^{m_{0}} c\left(\prod_{i=1}^{k} \delta_{i}\right)^{-1}
$$

for all $\boldsymbol{\delta} \in\left(0, \delta_{0}\right]^{m_{0}}$ by the assumed resolvent estimate and Lemma 4.2. And since

$$
\begin{gathered}
\left\|R_{\delta}(t)\right\|_{X \rightarrow X} \leq \sum_{k=0}^{m_{0}-1} \frac{1}{\delta^{k+1}}\left\|(A(t)-\lambda(t))^{k} P(t)\right\|_{X \rightarrow X}+\left\|\bar{R}_{\delta}(t)\right\|_{X \rightarrow X} \leq \frac{c}{\delta^{m_{0}}} \\
\quad \text { as well as } \\
\left\|\bar{R}_{\delta}(t)\right\|_{X \rightarrow Y} \leq\left\|(A(t)-1)^{-1}\right\|_{X \rightarrow Y}\left\|(A(t)-1) \bar{R}_{\delta}(t)\right\|_{X \rightarrow X} \leq \frac{c}{\delta}
\end{gathered}
$$

for all $t \in I$ and all $\delta \in\left(0, \delta_{0}\right]$ (with another constant $c$ ) by the assumed resolvent estimate and Lemma 4.2, it follows from Lemma 2.5 that there is a $W_{*}^{1, \infty}$-derivative $\bar{R}_{\delta}^{\prime}$ of $t \mapsto \bar{R}_{\delta}(t)$ such that

$$
\underset{t \in I}{\operatorname{ess-sup}}\left\|\bar{R}_{\delta}^{\prime}(t)\right\| \leq \frac{c}{\delta^{m_{0}+1}}
$$


for all $\delta \in\left(0, \delta_{0}\right]$ (with yet another constant $c$ ) and, hence, that there is a $W_{*}^{1, \infty}$-derivative $B_{n \delta}^{\prime}$ of $t \mapsto B_{n \delta}(t)$ such that

$$
\underset{t \in I}{\operatorname{ess}-\sup }\left\|B_{n \boldsymbol{\delta}}^{\prime}(t)\right\| \leq \sum_{k=1}^{m_{0}} c_{n}\left(\prod_{i=1}^{k} \delta_{i}\right)^{-\left(m_{0}+1\right)}
$$

for all $\boldsymbol{\delta} \in\left(0, \delta_{0}\right]^{m_{0}}$ and some constant $c_{n} \in(0, \infty)$ depending on the supremum norm $\sup _{t \in I}\left\|Q_{n}^{\prime}(t)\right\|$ of the SOT-derivative of $t \mapsto Q_{n}(t)$.

After these preparations we can now turn to the main part of the proof where the cases (i) and (ii) have to be treated separately. We first prove assertion (i). As has already been shown in (4.2),

$$
\left(V_{0 \varepsilon}(t)-U_{\varepsilon}(t)\right) P(0) x=\left.U_{\varepsilon}(t, s) V_{0 \varepsilon}(s) P(0) x\right|_{s=0} ^{s=t}=\int_{0}^{t} U_{\varepsilon}(t, s) P^{\prime}(s) V_{0 \varepsilon}(s) P(0) x d s
$$

so that, by rewriting the right hand side of this equation, we obtain

$$
\begin{aligned}
\left(V_{0 \varepsilon}(t)-U_{\varepsilon}(t)\right) P(0) x= & \int_{0}^{t} U_{\varepsilon}(t, s)\left(P^{\prime}(s)-Q_{n}(s)\right) P(s) V_{0 \varepsilon}(s) P(0) x d s \\
& +\int_{0}^{t} U_{\varepsilon}(t, s)\left[Q_{n}(s), P(s)\right] V_{0 \varepsilon}(s) P(0) x d s
\end{aligned}
$$

for all $t \in I, \varepsilon \in(0, \infty)$ and $x \in X$. Since $Q_{n}(s) P(s) \longrightarrow P^{\prime}(s) P(s)$ for every $s \in(0,1)$ by the SOT-convergence of $\left(Q_{n}(s)\right)$ to $P^{\prime}(s)$ for $s \in(0,1)$ and by $\operatorname{rk} P(s)=\operatorname{rk} P(0)<\infty$ for $s \in I$, it follows by Lemma 4.2 and by the dominated convergence theorem that

$$
\sup _{\varepsilon \in(0, \infty)} \sup _{t \in I}\left\|\int_{0}^{t} U_{\varepsilon}(t, s)\left(P^{\prime}(s)-Q_{n}(s)\right) P(s) V_{0 \varepsilon}(s) P(0) d s\right\| \longrightarrow 0
$$

as $n \rightarrow \infty$. In view of (4.10) we therefore have to show that for each fixed $n \in \mathbb{N}$

$$
\sup _{t \in I}\left\|\int_{0}^{t} U_{\varepsilon}(t, s)\left[Q_{n}(s), P(s)\right] V_{0 \varepsilon}(s) P(0) d s\right\| \longrightarrow 0
$$

as $\varepsilon \searrow 0$. So let $n \in \mathbb{N}$ be fixed for the rest of the proof. Since $s \mapsto B_{n \delta}(s)$ is in $W_{*}^{1, \infty}(I, L(X, Y))$ by the third preparatory step and since $\left.[0, t] \ni s \mapsto U_{\varepsilon}(t, s)\right|_{Y} \in$ $L(Y, X)$ as well as $s \mapsto V_{0 \varepsilon}(s) \in L(X)$ are SOT-continuously differentiable, Lemma 2.5 yields that

$$
[0, t] \ni s \mapsto U_{\varepsilon}(t, s) B_{n \boldsymbol{\delta}}(s) V_{0 \varepsilon}(s) P(0) x
$$

is the continuous representative of an element of $W^{1, \infty}([0, t], X)$ for every $x \in X$. With the help of the approximate commutator equation (4.5) of the second preparatory step, 
we therefore see that

$$
\begin{gathered}
\int_{0}^{t} U_{\varepsilon}(t, s)\left[Q_{n}(s), P(s)\right] V_{0 \varepsilon}(s) P(0) x d s=\varepsilon \int_{0}^{t} U_{\varepsilon}(t, s)\left(-\frac{1}{\varepsilon} A(s) B_{n} \boldsymbol{\delta}(s)\right. \\
\left.+B_{n \boldsymbol{\delta}}(s) \frac{1}{\varepsilon} A(s)\right) V_{0 \varepsilon}(s) P(0) x d s+\int_{0}^{t} U_{\varepsilon}(t, s) C_{n \boldsymbol{\delta}}^{+}(s) V_{0 \varepsilon}(s) P(0) x d s \\
=\left.\varepsilon U_{\varepsilon}(t, s) B_{n} \boldsymbol{\delta}(s) V_{0 \varepsilon}(s) P(0) x\right|_{s=0} ^{s=t}-\varepsilon \int_{0}^{t} U_{\varepsilon}(t, s)\left(B_{n \boldsymbol{\delta}}^{\prime}(s)+B_{n \boldsymbol{\delta}}(s)\left[P^{\prime}(s), P(s)\right]\right) \\
V_{0 \varepsilon}(s) P(0) x d s+\int_{0}^{t} U_{\varepsilon}(t, s) C_{n \boldsymbol{\delta}}^{+}(s) V_{0 \varepsilon}(s) P(0) x d s
\end{gathered}
$$

for all $t \in I, \varepsilon \in(0, \infty), x \in X$ and $\delta \in\left(0, \delta_{0}\right]^{m_{0}}$. We now want to find functions $\varepsilon \mapsto \delta_{1 \varepsilon}, \ldots, \delta_{m_{0} \varepsilon}$ defined on a small interval $\left(0, \delta_{0}^{\prime}\right]$ and converging to 0 as $\varepsilon \searrow 0$ in such a way that, if they are inserted in the right hand side of (4.13), the desired convergence (4.12) follows. In view of the estimates (4.7), (4.9) and

$$
\int_{0}^{1}\left\|C_{n \boldsymbol{\delta}}^{+}(s)\right\| d s \leq \sum_{k=1}^{m_{0}} c\left(\prod_{1 \leq i<k} \delta_{i}\right)^{-1} \int_{0}^{1}\left\|\delta_{k} \bar{R}_{\delta_{k}}(s) Q_{n}(s) P(s)\right\| d s,
$$

we would like the functions $\varepsilon \mapsto \delta_{i \varepsilon}$ to converge to 0 so slowly that

$$
\begin{gathered}
\varepsilon\left(\prod_{i=1}^{k} \delta_{i \varepsilon}\right)^{-\left(m_{0}+1\right)} \longrightarrow 0 \quad(\varepsilon \searrow 0) \\
\left(\prod_{1 \leq i<k} \delta_{i \varepsilon}\right)^{-1} \int_{0}^{1}\left\|\delta_{k \varepsilon} \bar{R}_{\delta_{k \varepsilon}}(s) Q_{n}(s) P(s)\right\| d s \longrightarrow 0 \quad(\varepsilon \searrow 0)
\end{gathered}
$$

for all $k \in\left\{1, \ldots, m_{0}\right\}$. Since

$$
\eta_{n}^{+}(\delta):=\int_{0}^{1}\left\|\delta \bar{R}_{\delta}(s) Q_{n}(s) P(s)\right\| d s \longrightarrow 0 \quad(\delta \searrow 0)
$$

by Lemma 4.1, by $\operatorname{rk} P(s)=\operatorname{rk} P(0)<\infty$ and by the dominated convergence theorem, such functions $\varepsilon \mapsto \delta_{i \varepsilon}$ really can be found. Indeed, define recursively

$$
\begin{gathered}
\delta_{m_{0} \varepsilon}:=\varepsilon^{\frac{1}{\left(m_{0}+1\right)^{2}}} \quad \text { and } \delta_{m_{0}-l \varepsilon}:=\max \left\{\left(\left(\prod_{m_{0}-l+1 \leq i<k} \delta_{i \varepsilon}\right)^{-1} \eta_{n}^{+}\left(\delta_{k \varepsilon}\right)\right)^{\frac{1}{2}}:\right. \\
\left.k \in\left\{m_{0}-l+1, \ldots, m_{0}\right\}\right\} \cup\left\{\varepsilon^{\frac{1}{\left(m_{0}+1\right)^{2}}}\right\}
\end{gathered}
$$

for $l \in\left\{1, \ldots, m_{0}-1\right\}$. With the help of (4.17) it then successively follows, by proceeding from larger to smaller indices $i$, that $\delta_{i \varepsilon} \longrightarrow 0$ as $\varepsilon \searrow 0$ for all $i \in\left\{1, \ldots, m_{0}\right\}$ (so that, in particular, $\delta_{i \varepsilon} \in\left(0, \delta_{0}\right]$ for small enough $\varepsilon$ whence the expressions $\eta_{n}^{+}\left(\delta_{i \varepsilon}\right)$ used in the recursive definition make sense for small $\varepsilon$ in the first place) and that (4.15) and (4.16) are satisfied. Assertion (i) now follows from (4.10), (4.11), (4.13) by virtue of (4.7), (4.9), (4.14) and Lemma 4.2. 
We now prove assertion (ii) and, for that purpose, additionally assume that $X$ is reflexive and $t \mapsto P(t)$ is NOT-continuously differentiable. Analogously to (4.10) we obtain

$$
\begin{aligned}
\left(V_{\varepsilon}(t)-U_{\varepsilon}(t)\right) x= & \int_{0}^{t} U_{\varepsilon}(t, s)\left[P^{\prime}(s)-Q_{n}(s), P(s)\right] V_{\varepsilon}(s) x d s \\
& +\int_{0}^{t} U_{\varepsilon}(t, s)\left[Q_{n}(s), P(s)\right] V_{\varepsilon}(s) x d s
\end{aligned}
$$

for all $t \in I, \varepsilon \in(0, \infty)$ and $x \in D(A(0))=D$. Since $Q_{n}(s) \longrightarrow P^{\prime}(s)$ for every $s \in(0,1)$ by the additionally assumed NOT-continuous differentiability of $t \mapsto P(t)$, it follows by Proposition 2.13 and by the dominated convergence theorem that

$$
\sup _{\varepsilon \in(0, \infty)} \sup _{t \in I}\left\|\int_{0}^{t} U_{\varepsilon}(t, s)\left[P^{\prime}(s)-Q_{n}(s), P(s)\right] V_{\varepsilon}(s) d s\right\| \longrightarrow 0
$$

as $n \rightarrow \infty$. In view of (4.18) we therefore have to show that for each fixed $n \in \mathbb{N}$

$$
\sup _{t \in I}\left\|\int_{0}^{t} U_{\varepsilon}(t, s)\left[Q_{n}(s), P(s)\right] V_{\varepsilon}(s) d s\right\| \longrightarrow 0
$$

as $\varepsilon \searrow 0$. So let $n \in \mathbb{N}$ be fixed for the rest of the proof. Again completely analogously to the proof of (i) it follows that

$$
[0, t] \ni s \mapsto U_{\varepsilon}(t, s) B_{n \delta}(s) V_{\varepsilon}(s) x
$$

is the continuous representative of an element of $W^{1, \infty}([0, t], X)$ for every $x \in D(A(0))=$ $D$. With the help of the approximate commutator equation (4.5) of the second preparatory step, we therefore see that

$$
\begin{gathered}
\int_{0}^{t} U_{\varepsilon}(t, s)\left[Q_{n}(s), P(s)\right] V_{\varepsilon}(s) x d s=\frac{1}{\varepsilon} \int_{0}^{t} U_{\varepsilon}(t, s)\left(-\frac{1}{\varepsilon} A(s) B_{n} \boldsymbol{\delta}(s)\right. \\
\left.+B_{n \boldsymbol{\delta}}(s) \frac{1}{\varepsilon} A(s)\right) V_{\varepsilon}(s) x d s+\int_{0}^{t} U_{\varepsilon}(t, s) C_{n} \boldsymbol{\delta}(s) V_{\varepsilon}(s) x d s \\
=\left.\varepsilon U_{\varepsilon}(t, s) B_{n} \boldsymbol{\delta}(s) V_{\varepsilon}(s) x\right|_{s=0} ^{s=t}-\varepsilon \int_{0}^{t} U_{\varepsilon}(t, s)\left(B_{n \boldsymbol{\delta}}^{\prime}(s)+B_{n \boldsymbol{\delta}}(s)\left[P^{\prime}(s), P(s)\right]\right) \\
V_{\varepsilon}(s) x d s+\int_{0}^{t} U_{\varepsilon}(t, s) C_{n \boldsymbol{\delta}}(s) V_{\varepsilon}(s) x d s
\end{gathered}
$$

for all $t \in I, \varepsilon \in(0, \infty), x \in D(A(0))=D$ and $\boldsymbol{\delta} \in\left(0, \delta_{0}\right]^{m_{0}}$. In view of the estimates (4.7), (4.9), (4.14) and

$$
\int_{0}^{1}\left\|C_{n \boldsymbol{\delta}}^{-}(s)\right\| d s \leq \sum_{k=1}^{m_{0}} c\left(\prod_{1 \leq i<k} \delta_{i}\right)^{-1} \int_{0}^{1}\left\|P(s) Q_{n}(s) \delta_{k} \bar{R}_{\delta_{k}}(s)\right\| d s
$$


we would now like to find functions $\varepsilon \mapsto \delta_{1 \varepsilon}, \ldots, \delta_{m_{0} \varepsilon}$ defined on a small interval $\left(0, \delta_{0}^{\prime}\right]$ and converging to 0 as $\varepsilon \searrow 0$ so slowly that (4.15), (4.16) and

$$
\left(\prod_{1 \leq i<k} \delta_{i \varepsilon}\right)^{-1} \int_{0}^{1}\left\|P(s) Q_{n}(s) \delta_{k \varepsilon} \bar{R}_{\delta_{k \varepsilon}}(s)\right\| d s \longrightarrow 0 \quad(\varepsilon \searrow 0)
$$

are satisfied for all $k \in\left\{1, \ldots, m_{0}\right\}$. Why is it possible to find such functions $\varepsilon \mapsto \delta_{i \varepsilon}$ ? In essence, this is because of (4.17) and because

$$
\eta_{n}^{-}(\delta):=\int_{0}^{1}\left\|P(s) Q_{n}(s) \delta \bar{R}_{\delta}(s)\right\| d s \longrightarrow 0 \quad(\delta \searrow 0),
$$

which last convergence can be seen as follows: by virtue of Proposition 2.2, which applies by the additionally assumed reflexivity of $X, P(s)^{*}$ is weakly $m_{0}$-associated with $A(s)^{*}$ and $\lambda(s)$ for almost every $s \in I$, and therefore Lemma 4.1 together with $\operatorname{rk} P(s)^{*}=$ rk $P(s)<\infty$ yields the convergence

$$
\left\|P(s) Q_{n}(s) \delta \bar{R}_{\delta}(s)\right\|=\left\|\delta \bar{R}_{\delta}(s)^{*} Q_{n}(s)^{*} P(s)^{*}\right\| \longrightarrow 0 \quad(\delta \searrow 0)
$$

for almost every $s \in I$, from which (4.24) follows by the dominated convergence theorem. We now recursively define

$$
\begin{gathered}
\delta_{m_{0} \varepsilon}:=\varepsilon^{\frac{1}{\left(m_{0}+1\right)^{2}}} \text { and } \delta_{m_{0}-l \varepsilon}:=\max \left\{\left(\left(\prod_{m_{0}-l+1 \leq i<k} \delta_{i \varepsilon}\right)^{-1} \eta_{n}^{+}\left(\delta_{k \varepsilon}\right)\right)^{\frac{1}{2}},\right. \\
\left.\left(\left(\prod_{m_{0}-l+1 \leq i<k} \delta_{i \varepsilon}\right)^{-1} \eta_{n}^{-}\left(\delta_{k \varepsilon}\right)\right)^{\frac{1}{2}}: k \in\left\{m_{0}-l+1, \ldots, m_{0}\right\}\right\} \cup\left\{\varepsilon^{\frac{1}{\left(m_{0}+1\right)^{2}}}\right\}
\end{gathered}
$$

for $l \in\left\{1, \ldots, m_{0}-1\right\}$. With the help of (4.17) and (4.24) it then successively follows, by proceeding from larger to smaller indices $i$, that $\delta_{i \varepsilon} \longrightarrow 0$ as $\varepsilon \searrow 0$ for all $i \in\left\{1, \ldots, m_{0}\right\}$ and that (4.15), (4.16) and (4.23) are satisfied. Assertion (ii) now follows from (4.18), (4.19), (4.21) by virtue of (4.7), (4.9), (4.14), (4.22) and Proposition 2.13.

Some remarks, which in particular clarify the relation of the above theorem with the adiabatic theorem without spectral gap condition from [8] and [46], are in order.

1. Clearly, the adiabatic theorem above generalizes the adiabatic theorems without spectral gap condition from [8] (Theorem 11) and [46] (Theorem 6.4) which cover the less general case of weakly semisimple eigenvalues $\lambda(t)$ of (not necessarily skew selfadjoint) operators $A(t): D \subset X \rightarrow X$ under less general regularity conditions. See Section 4.3 for simple examples where the previously known adiabatic theorems cannot be applied - and which show, moreover, that the adiabatic theorem above is by no means confined to spectral operators. In the special case where the eigenvalues $\lambda(t)$ from the above theorem lie on the imaginary axis $i \mathbb{R}$ for every $t \in I$, these eigenvalues are automatically weakly semisimple by the $(M, 0)$-stability hypothesis of the theorem, which by Joye's example (from the end of Section 3.3) cannot be essentially weakened, and by the weak associatedness hypothesis. (Argue as in the second remark at the beginning 
of Section 3.3.) And so, the above adiabatic theorem - in the special case of purely imaginary eigenvalues - essentially reduces to the adiabatic theorems without spectral gap condition from [8] and [46] - but a general adiabatic theory without spectral gap condition should be able to cover more than just this special case, of course.

2. As can be seen from the proof of the theorem above, one would obtain the same conclusion if - instead of requiring the weak associatedness of $P(t)$ with $A(t)$ and $\lambda(t)$ for almost every $t \in I$ - one only required the inclusions

$$
P(t) X \subset \operatorname{ker}(A(t)-\lambda(t))^{m_{0}} \quad \text { and } \quad(1-P(t)) X \subset \overline{\operatorname{rg}(A(t)-\lambda(t))^{m_{0}}}
$$

for almost every $t \in I$ and some $m_{0} \in \mathbb{N}$. We point out, however, that one would nevertheless not obtain a truly more general theorem by thus modifying the hypotheses, because the projections $P(t)$ would then still be weakly associated with $A(t)$ and $\lambda(t)$ for almost every $t \in I$. (In order to see this, notice that, for every $t \in I$ where (4.25) is satisfied, $(1-P(t)) \operatorname{ker}(A(t)-\lambda(t))^{m_{0}}=0$ by Lemma 4.1 and an expansion similar to (3.7) and that $P(t) \operatorname{rg}(A(t)-\lambda(t))^{m_{0}}=0$ so that $P(t) \overline{\operatorname{rg}(A(t)-\lambda(t))^{m_{0}}}=0$ as well. And then apply the first remark after Theorem 2.1.) Also it can be seen from the proof of the above theorem: if the finite rank hypothesis on $P(0)$ is the only one to be violated, one still has the strong convergence

$$
\sup _{t \in I}\left\|\left(U_{\varepsilon}(t)-V_{0 \varepsilon}(t)\right) P(0) x\right\| \longrightarrow 0 \quad(\varepsilon \searrow 0) \quad \text { for every } x \in X,
$$

provided $P(t)$ is even weakly 1-associated with $A(t)$ and $\lambda(t)$ for almost every $t \in I$. (In order to see this, notice that, under this extra condition, the inclusion $P(t) X \subset$ $\operatorname{ker}(A(t)-\lambda(t))$ holds for every $t \in I$ by a closedness argument similar to the one in (3.5) and the $\varepsilon$-dependence of $V_{0 \varepsilon}(s) P(0)$ is solely contained in a scalar factor,

$$
V_{0 \varepsilon}(s) P(0)=e^{\frac{1}{\varepsilon} \int_{0}^{s} \lambda(\tau) d \tau} W(s) P(0) \quad(s \in I),
$$

where $W$ denotes the evolution system for $\left[P^{\prime}, P\right]$.)

3. As in the case with spectral gap, the adiabatic theorem without spectral gap condition above can - along with the approximate commutator equation method used in its proof - be extended to several eigenvalues $\lambda_{1}(t), \ldots, \lambda_{r}(t)$ : if $A, \lambda_{j}, P_{j}$ for every $j \in\{1, \ldots, r\}$ satisfy the hypotheses of part (ii) of the above adiabatic theorem and if $\lambda_{j}($.$) and \lambda_{l}($.$) for all j \neq l$ fall into each other at only countably many points accumulating at only finitely many points, then the evolution system $V_{\varepsilon}$ for $\frac{1}{\varepsilon} A+K$ with $K$ as in (3.8) is adiabatic w.r.t. all the $P_{j}$ and well approximates the evolution system $U_{\varepsilon}$ for $\frac{1}{\varepsilon} A$ in the sense that

$$
\sup _{t \in I}\left\|U_{\varepsilon}(t)-V_{\varepsilon}(t)\right\| \longrightarrow 0 \quad(\varepsilon \searrow 0),
$$

provided $V_{\varepsilon}$ exists on $D$. In order to see this (in the technically simpler case where the $t \mapsto P_{j}(t)$ are twice continuously SOT-differentiable), one sets $B_{\boldsymbol{\delta}}(t):=\frac{1}{2} \sum_{j=1}^{r+1} B_{j} \boldsymbol{\delta}(t)$ 
where

$$
\begin{gathered}
B_{j \delta}:=\sum_{k=0}^{m_{0}-1}\left(\prod_{i=1}^{k+1} \bar{R}_{j} \delta_{i}\right) P_{j}^{\prime} P_{j}\left(\lambda_{j}-A\right)^{k}+\sum_{k=0}^{m_{0}-1}\left(\lambda_{j}-A\right)^{k} P_{j} P_{j}^{\prime}\left(\prod_{i=1}^{k+1} \bar{R}_{j} \delta_{i}\right)(j \in\{1, \ldots, r\}) \\
\text { with } \bar{R}_{j \delta}:=\left(\lambda_{j}+\delta e^{i \vartheta_{j}}-A\right)^{-1}\left(1-P_{j}\right) \text { and where } \\
B_{r+1 \delta}:=\sum_{j=1}^{r} B_{j} \delta+\sum_{j \neq l} B_{j l}, \\
B_{j l}:=\sum_{i, k=0}^{m_{0}-1}\left(\begin{array}{c}
k+i \\
i
\end{array}\right) \frac{(-1)^{i}}{\left(\lambda_{l}-\lambda_{j}\right)^{k+i+1}}\left(\left(A-\lambda_{j}\right)^{k} P_{j} P_{j}^{\prime} P_{l}\left(A-\lambda_{l}\right)^{i}\right. \\
\left.+\left(A-\lambda_{l}\right)^{i} P_{l} P_{j}^{\prime} P_{j}\left(A-\lambda_{j}\right)^{k}\right)
\end{gathered}
$$

and then one shows that the approximate commutator equation $B_{\boldsymbol{\delta}}(t) A(t)-A(t) B_{\boldsymbol{\delta}}(t) \subset$ $K(t)-C_{\boldsymbol{\delta}}(t)$ with $C_{\boldsymbol{\delta}}(t):=\sum_{j=1}^{r} C_{j \boldsymbol{\delta}}(t)$ and

$C_{j \delta}:=\sum_{k=0}^{m_{0}-1} \delta_{k+1} e^{i \vartheta_{j}}\left(\prod_{i=1}^{k+1} \bar{R}_{j} \delta_{i}\right) P_{j}^{\prime} P_{j}\left(\lambda_{j}-A\right)^{k}-\sum_{k=0}^{m_{0}-1}\left(\lambda_{j}-A\right)^{k} P_{j} P_{j}^{\prime} \delta_{k+1} e^{i \vartheta_{j}}\left(\prod_{i=1}^{k+1} \bar{R}_{j} \delta_{i}\right)$

is satisfied for all points $t$ where $P_{j}(t)$ is weakly associated with $A(t), \lambda_{j}(t)$ for every $j \in\{1, \ldots, r\}$ and where no crossing between two curves $\lambda_{j}($.$) and \lambda_{l}($.$) takes place -$ by showing that for all such $t$ and all $j \neq l$

$$
B_{j l}(t) A(t)-A(t) B_{j l}(t) \subset P_{j}(t) P_{j}^{\prime}(t) P_{l}(t)-P_{l}(t) P_{j}^{\prime}(t) P_{j}(t)=\left[P_{j}^{\prime}(t), P_{l}(t)\right]
$$

(for the second equality, use that $P_{j}(t) P_{l}(t)=0=P_{l}(t) P_{j}(t)$ which is due to the weak associatedness of $P_{j}(t), P_{l}(t)$ with $A(t), \lambda_{j}(t)$ resp. $\lambda_{l}(t)$ and to $\left.\lambda_{j}(t) \neq \lambda_{l}(t)\right)$ and by noticing that $\left[P_{r+1}^{\prime}, P_{r+1}\right]=\left[P^{\prime}, P\right]$. At first glance, the defining formula for $B_{r+1} \delta$ might seem a bit mysterious, but in fact it can be guessed, just like the formulas for $B_{1} \boldsymbol{\delta}, \ldots, B_{r} \boldsymbol{\delta}$, from the case with spectral gap: indeed, by rewriting the formula for $B_{r+1}$ from (3.9) - in the special case of singletons $\sigma_{j}(t)=\left\{\lambda_{j}(t)\right\}$ consisting of poles of $(.-A(t))^{-1}$ of order at most $m_{0}$ - one obtains $B_{r+1}=\left.B_{r+1} \delta\right|_{\delta=0}$ with the help of Cauchy's theorem.

\subsection{A quantitative adiabatic theorem without spectral gap condition - especially for scalar type spectral operators}

As a supplement to the qualitative adiabatic theorem above (Theorem 4.3), we note the following quantitative refinement. It implies that, if in the situation of the above theorem the map $t \mapsto P(t)$ is even $W_{*}^{2, \infty}$-regular, then the rate of convergence (Lemma 4.1.) of the integrals

$$
\begin{aligned}
\eta^{+}(\delta):=\int_{0}^{1} \| \delta(\lambda(s) & \left.+\delta e^{i \vartheta(s)}-A(s)\right)^{-1} P^{\prime}(s) P(s) \| d s \\
\eta^{-}(\delta) & :=\int_{0}^{1}\left\|P(s) P^{\prime}(s) \delta\left(\lambda(s)+\delta e^{i \vartheta(s)}-A(s)\right)^{-1}\right\| d s
\end{aligned}
$$


yields a simple upper bound on the rate of convergence of $\sup _{t \in I}\left\|U_{\varepsilon}(t)-V_{\varepsilon}(t)\right\|$ which we are interested in here. See [52] for an analogous result in the case of skew self-adjoint operators $A(t)$.

Theorem 4.4. Suppose that $A(t), \lambda(t), P(t)$ are as in Theorem 4.3 with $X$ not necessarily reflexive and that $t \mapsto P(t)$ is even in $W_{*}^{2, \infty}(I, L(X))$. Suppose further that $\eta:\left(0, \delta_{0}\right] \subset(0,1] \rightarrow(0, \infty)$ is a function such that $\eta(\delta) \longrightarrow 0$ as $\delta \searrow 0$ and

$$
\eta(\delta) \geq \delta \quad \text { as well as } \eta^{ \pm}(\delta) \leq \eta(\delta)
$$

for all $\delta \in\left(0, \delta_{0}\right]$ with $\eta^{ \pm}$as above. Then there is a constant $c$ such that

$$
\sup _{t \in I}\left\|U_{\varepsilon}(t)-V_{\varepsilon}(t)\right\| \leq c \tilde{\eta}^{m_{0}}\left(\varepsilon^{2 /\left(m_{0}\left(m_{0}+1\right)\right)}\right)=c(\tilde{\eta} \circ \cdots \circ \tilde{\eta})\left(\varepsilon^{2 /\left(m_{0}\left(m_{0}+1\right)\right)}\right)
$$

for $\varepsilon$ sufficiently small, where $\tilde{\eta}(\delta):=\eta\left(\delta^{\frac{1}{2}}\right)$.

Proof. We proceed as in the proof of the qualitative adiabatic theorem above, but now replace $Q_{n}$ and $Q_{n}^{\prime}$ at any occurrence by $P^{\prime}$ and $P^{\prime \prime}$. We can then conclude from (4.18) and (4.21) (with the replacements just mentioned) that there is a constant $c^{\prime}$ such that

$$
\begin{aligned}
& \sup _{t \in I}\left\|U_{\varepsilon}(t)-V_{\varepsilon}(t)\right\| \leq \\
& \quad c^{\prime}\left(\sum_{k=1}^{m_{0}} \varepsilon\left(\prod_{j=1}^{m_{0}} \delta_{j}\right)^{-1}+\sum_{k=1}^{m_{0}} \varepsilon\left(\delta_{k}^{m_{0}+1} \prod_{j \neq k} \delta_{j}\right)^{-1} \eta\left(\delta_{k}\right)+\sum_{k=1}^{m_{0}}\left(\prod_{1 \leq j<k} \delta_{j}\right)^{-1} \eta\left(\delta_{k}\right)\right)
\end{aligned}
$$

for all $\delta_{1}, \ldots, \delta_{m_{0}} \in\left(0, \delta_{0}\right]$ and $\varepsilon \in(0, \infty)$. In this estimate the first, second, and third sum correspond to the $B_{\delta^{-}}, B_{\delta^{-}}^{\prime}, C_{\delta^{-}}$terms in (4.21), respectively. (See (4.7) and (4.14), (4.22) for the estimation of the $B_{\delta}$-terms and $C_{\delta}$-terms. And to obtain the upper bound for the $B_{\boldsymbol{\delta}}^{\prime}$-terms, refine the estimate on $\int_{0}^{1}\left\|B_{\boldsymbol{\delta}}^{\prime}(s)\right\| d s$ from the proof of the previous theorem by using - instead of (4.9) - the fact that

$$
\int_{0}^{1}\left\|P(s) P^{\prime}(s) \bar{R}_{\delta}(s)\right\| d s, \int_{0}^{1}\left\|(A(s)-1) \bar{R}_{\delta}(s) P^{\prime}(s) P(s)\right\| d s \leq c \frac{\eta(\delta)}{\delta}
$$

and that $\sup _{s \in I}\left\|R_{\delta}(s)\right\|, \sup _{s \in I}\left\|(A(s)-1) R_{\delta}(s)\right\| \leq \frac{c}{\delta^{m} 0}$ for all sufficiently small $\delta \in$ $\left(0, \delta_{0}\right]$.) We now recursively define

$$
\delta_{m_{0} \varepsilon}:=\varepsilon^{\frac{1}{m_{0}\left(m_{0}+1\right)}} \quad \text { and } \quad \delta_{m_{0}-k \varepsilon}:=\left(\eta\left(\delta_{m_{0}-k+1 \varepsilon}\right)\right)^{\frac{1}{2}}
$$

for $\varepsilon$ so small that $\delta_{m_{0}-k+1 \varepsilon}$ lies in $\left(0, \delta_{0}\right]$ and for $k \in\left\{1, \ldots, m_{0}-1\right\}$. (It should be noticed that $\delta_{m_{0}-k+1 \varepsilon} \longrightarrow 0$ as $\varepsilon \searrow 0$ because $\eta(\delta) \longrightarrow 0$ and that $\delta_{m_{0}-k+1 \varepsilon}$ therefore really lies in the domain $\left(0, \delta_{0}\right]$ of $\eta$ for sufficiently small $\varepsilon$.) Since $\eta\left(\delta_{1 \varepsilon}\right)=\tilde{\eta}^{m_{0}}\left(\varepsilon^{2 /\left(m_{0}\left(m_{0}+1\right)\right)}\right)$ and $\frac{1}{\delta_{k-1 \varepsilon}} \eta\left(\delta_{k \varepsilon}\right)=\delta_{k-1 \varepsilon} \leq \eta\left(\delta_{k-1 \varepsilon}\right)$ for $k \in\left\{2, \ldots, m_{0}\right\}$, it follows by induction that

$$
\left(\prod_{1 \leq j<k} \delta_{j \varepsilon}\right)^{-1} \eta\left(\delta_{k \varepsilon}\right) \leq \tilde{\eta}^{m_{0}}\left(\varepsilon^{2 /\left(m_{0}\left(m_{0}+1\right)\right)}\right)
$$


and, in particular, $\eta\left(\delta_{k \varepsilon}\right) \leq \tilde{\eta}^{m_{0}}\left(\varepsilon^{2 /\left(m_{0}\left(m_{0}+1\right)\right)}\right)$ for all $k \in\left\{1, \ldots, m_{0}\right\}$ and sufficiently small $\varepsilon$. Since $\delta_{m_{0} \varepsilon} \leq \delta_{m_{0}-k+1 \varepsilon} \leq \delta_{m_{0}-k \varepsilon}$ for $k \in\left\{1, \ldots, m_{0}-1\right\}$ and small $\varepsilon$, it further follows that

$$
\begin{aligned}
\varepsilon\left(\prod_{j=1}^{k} \delta_{j \varepsilon}\right)^{-1} \leq \varepsilon\left(\delta_{k \varepsilon}^{m_{0}+1} \prod_{j \neq k} \delta_{j \varepsilon}\right)^{-1} \eta\left(\delta_{k \varepsilon}\right) & \leq \varepsilon\left(\prod_{j=1}^{m_{0}} \delta_{m_{0} \varepsilon}\right)^{-\left(m_{0}+1\right)} \tilde{\eta}^{m_{0}}\left(\varepsilon^{2 /\left(m_{0}\left(m_{0}+1\right)\right)}\right) \\
& =\tilde{\eta}^{m_{0}}\left(\varepsilon^{2 /\left(m_{0}\left(m_{0}+1\right)\right)}\right)
\end{aligned}
$$

for all $k \in\left\{1, \ldots, m_{0}\right\}$ and sufficiently small $\varepsilon$. Combining (4.27), (4.28) and (4.29) we finally obtain the assertion.

1. Clearly, a function $\eta$ as described in the above theorem exists under the hypotheses of the qualitative adiabatic theorem (Theorem 4.3) including the reflexivity of $X$. In fact, one has only to define $\eta(\delta):=\max \left\{\eta^{+}(\delta), \eta^{-}(\delta), \delta\right\}$ with $\eta_{ \pm}$as in (4.26) and to remember that $\eta(\delta) \longrightarrow 0$ as $\delta \searrow 0$ by (4.17) and (4.24).

2. An inspection of the proof above - or, more precisely, of the arguments leading to (4.27) - shows that one obtains the same conclusion if one drops the finite rank hypothesis on $P(0)$ and, at the same time, replaces the hypothesis that $P(t)$ be weakly associated with $A(t)$ and $\lambda(t)$ for almost every $t \in I$ by the condition that there exist an $m_{0} \in \mathbb{N}$ such that

$$
P(t) X \subset \operatorname{ker}(A(t)-\lambda(t))^{m_{0}}
$$

for every $t \in I$ (while leaving all other hypotheses of the above theorem unchanged).

We now specialize to the case of spectral operators of scalar type which - by what has been remarked in Section 2.1 - automatically have weakly semisimple eigenvalues and which arise as the generic one-dimensional periodic Schrödinger operators (Remark 8.7 of [22]). As a simple corollary of the adiabatic theorem above, we obtain the following quantitative adiabatic theorem tailored to scalar type spectral operators $A(t)$ whose spectral measures $P^{A(t)}$ are - in some sense - Hölder continuous in $t$. It generalizes a result for skew self-adjoint $A(t)$ of Avron and Elgart (Corollary 1 in [7]) and a refinement of it due to Teufel (Remark 1 in [52]) and slightly improves the rates of convergence given there.

Corollary 4.5. Suppose $A(t): D \subset X \rightarrow X$ for every $t \in I$ is a spectral operator of scalar type (with spectral measure $P^{A(t)}$ ) such that Condition 2.10 is satisfied with $\omega=0$ and such that $\sup _{t \in I} \sup _{E \in \mathcal{B}_{\mathbb{C}}}\left\|P^{A(t)}(E)\right\|<\infty$. Suppose further that $\lambda(t)$ for every $t \in I$ is an eigenvalue of $A(t)$ such that the open sector

$$
\lambda(t)+\delta_{0} S_{\left(\vartheta(t)-\vartheta_{0}, \vartheta(t)+\vartheta_{0}\right)}:=\left\{\lambda(t)+\delta e^{i \vartheta}: \delta \in\left(0, \delta_{0}\right), \vartheta \in\left(\vartheta(t)-\vartheta_{0}, \vartheta(t)+\vartheta_{0}\right)\right\}
$$

of radius $\delta_{0} \in(0, \infty)$ and angle $2 \vartheta_{0} \in(0, \pi)$ for every $t \in I$ is contained in $\rho(A(t))$ and such that $t \mapsto \lambda(t), e^{i \vartheta(t)}$ are Lipschitz continuous. Suppose finally that $P(t)$ for every $t \in I$ is a bounded projection in $X$ such that $P(t)=P^{A(t)}(\{\lambda(t)\})$ for almost every $t \in I$ 
and $t \mapsto P(t)$ is in $W_{*}^{2, \infty}(I, L(X))$, and suppose that $P^{A(t)}$ is Hölder continuous with exponent $\alpha \in(0,1]$ uniformly in $t \in I$, that is, there is a $c_{0} \in(0, \infty)$ such that

$$
\left\|P^{A(t)}(E) x\right\| \leq c_{0} \lambda(E)^{\frac{\alpha}{2}}\|x\|
$$

for all $E \in \mathcal{B}_{\mathbb{C}}$ with $\lambda(E) \leq 1$ and all $x \in X$ and $t \in I$. Then there is a constant $c \in(0, \infty)$ such that

$$
\sup _{t \in I}\left\|U_{\varepsilon}(t)-V_{\varepsilon}(t)\right\| \leq c \varepsilon^{\frac{\alpha}{2(1+\alpha)}}
$$

for small enough $\varepsilon \in(0, \infty)$, where $V_{\varepsilon}$ denotes the evolution system for $\frac{1}{\varepsilon} A+\left[P^{\prime}, P\right]$.

Proof. We show that there exists a function $\eta:\left(0, \delta_{0}^{\prime}\right] \rightarrow(0, \infty)$ such that $\eta(\delta) \longrightarrow 0$ as $\delta \searrow 0$ and

$$
\eta(\delta) \geq \delta \quad \text { as well as } \quad\left\|\delta R_{\delta}(t)\right\|=\left\|\delta\left(\lambda(t)+\delta e^{i \vartheta(t)}-A(t)\right)^{-1}\right\| \leq \eta(\delta)
$$

for all $\delta \in\left(0, \delta_{0}^{\prime}\right]$ and $t \in I$ (with a suitable $\left.\delta_{0}^{\prime}\right)$. In order to do so, we notice that, by the scalar type spectrality of $A(t)$ and Theorem XVIII.2.11 of [18],

$$
\left|\left\langle x^{*}, \delta R_{\delta}(t) x\right\rangle\right| \leq \int_{\sigma(A(t))} \frac{\delta}{\left|\lambda(t)+\delta e^{i \vartheta(t)}-z\right|} d\left|P_{x^{*}, x}^{A(t)}\right|(z),
$$

where $\left|P_{x *, x}^{A(t)}\right|$ denotes the total variation of the complex measure $E \mapsto P_{x^{*}, x}^{A(t)}(E):=$ $\left\langle x^{*}, P^{A(t)}(E) x\right\rangle$, and then divide the spectrum $\sigma(A(t))$ of $A(t)$ into the parts

$$
\sigma_{1 r_{\delta}}(t):=\sigma(A(t)) \cap U_{r_{\delta}}(\lambda(t)) \quad \text { and } \quad \sigma_{2 r_{\delta}}(t):=\sigma(A(t)) \cap \mathbb{C} \backslash U_{r_{\delta}}(\lambda(t))
$$

of those spectral values that are close to $\lambda(t)$ resp. far from $\lambda(t)$, where $r_{\delta}:=\delta^{\gamma}$ and $\gamma \in(0,1)$ will be chosen in (4.32) below. Since, by Lemma III.1.5 of [18],

$$
\left|P_{x^{*}, x}^{A(t)}\right|(E) \leq 4 \sup _{F \in \mathcal{B}_{E}}\left|\left\langle x^{*}, P^{A(t)}(F) P^{A(t)}(E) x\right\rangle\right| \leq 4 M^{\prime}\left\|x^{*}\right\|\left\|P^{A(t)}(E) x\right\|
$$

for every $t \in I$ and $E \in \mathcal{B}_{\mathbb{C}}$ (where $M^{\prime}:=\sup _{t \in I} \sup _{F \in \mathcal{B}_{\mathbb{C}}}\left\|P^{A(t)}(F)\right\|<\infty$ ) and since, by the assumed sector condition,

$$
\operatorname{dist}\left(\lambda(t)+\delta e^{i \vartheta(t)}, \sigma(A(t))\right) \geq\left(\sin \vartheta_{0}\right) \delta
$$

for every $t \in I$ and $\delta \in\left(0, \delta_{0}^{\prime}\right]$ (where $\delta_{0}^{\prime}$ is chosen small enough), there are positive constants $c_{1}, c_{2}$ such that

$$
\int_{\sigma_{1 r_{\delta}}(t)} \frac{\delta}{\left|\lambda(t)+\delta e^{i \vartheta(t)}-z\right|} d\left|P_{x^{*}, x}^{A(t)}\right|(z) \leq \frac{1}{\sin \vartheta_{0}}\left|P_{x^{*}, x}^{A(t)}\right|\left(U_{r_{\delta}}(\lambda(t))\right) \leq c_{1} \delta^{\alpha \gamma}\left\|x^{*}\right\|\|x\|
$$

as well as

$$
\int_{\sigma_{2 r_{\delta}}(t)} \frac{\delta}{\left|\lambda(t)+\delta e^{i \vartheta(t)}-z\right|} d\left|P_{x^{*}, x}^{A(t)}\right|(z) \leq \frac{\delta}{r_{\delta}-\delta}\left|P_{x^{*}, x}^{A(t)}\right|(\mathbb{C}) \leq c_{2} \delta^{1-\gamma}\left\|x^{*}\right\|\|x\|
$$


for every $x \in X, x^{*} \in X^{*}, \delta \in\left(0, \delta_{0}^{\prime}\right]$ and $t \in I$. Consequently,

$$
\left\|\delta R_{\delta}(t)\right\| \leq c_{1} \delta^{\alpha \gamma}+c_{2} \delta^{1-\gamma} \leq \max \left\{c_{1}, c_{2}\right\} \delta^{\min \{\alpha \gamma, 1-\gamma\}}=c_{0}^{\prime} \delta^{\beta(\gamma)}
$$

for every $t \in I$ and $\delta \in\left(0, \delta_{0}^{\prime}\right]$ (notice that $\beta(\gamma):=\min \{\alpha \gamma, 1-\gamma\}$, for given $\gamma$, is the best - that is, biggest - possible exponent in the second inequality above). And as $\gamma \mapsto \beta(\gamma)$ is maximal at $\gamma_{0}:=\frac{1}{1+\alpha}$, we choose

$$
\gamma:=\gamma_{0}, \quad \beta:=\beta\left(\gamma_{0}\right)=\frac{\alpha}{1+\alpha}, \quad \eta(\delta):=c_{0}^{\prime} \delta^{\beta}=c_{0}^{\prime} \delta^{\frac{\alpha}{1+\alpha}},
$$

thereby obtaining (4.30). Since $P(t) A(t) \subset A(t) P(t)=\lambda(t) P(t)$ holds for every $t \in I$ (by the scalar type spectrality of $A(t)$ and the closedness argument in the remark after Theorem 3.2), the desired conclusion follows by the second remark after Theorem 4.4 with $m_{0}=1$.

\subsection{Some examples}

We begin with two examples where $\lambda(t)$ is an eigenvalue of $A(t)$ that is allowed to be non-isolated and non-weakly-semisimple for every $t \in I$. In particular, these examples cannot be dealt with by way of the previously known adiabatic theorems. In the first example, $A(t)$ is a spectral operator whereas in the second it is not (by Theorem XV.3.10 and XV.8.7 of [18] and by the spectral structure of the right shift $S_{+}$(Section 2.4)).

Example 4.6. Suppose $A, \lambda, P$ with $A(t)=R(t)^{-1} A_{0}(t) R(t), P(t)=R(t)^{-1} P_{0} R(t)$, and $R(t)=e^{C t}$ are given as follows in $X:=\ell^{p}\left(I_{d}\right) \times \ell^{p}\left(I_{\infty}\right)($ where $p \in[1, \infty)$ and $d \in \mathbb{N}$ ):

$$
A_{0}(t):=\left(\begin{array}{cc}
\lambda(t)+\alpha(t) N & 0 \\
0 & \operatorname{diag}\left(\left(\lambda_{n}\right)_{n \in \mathbb{N}}\right)
\end{array}\right) \quad \text { and } \quad P_{0}:=\left(\begin{array}{ll}
1 & 0 \\
0 & 0
\end{array}\right),
$$

where $\lambda(t) \in(-\infty, 0], \alpha(t), N$ are such that Condition 2.16 is satisfied and where $\left(\lambda_{n}\right)_{n \in \mathbb{N}}$ is an enumeration of $[-1,0] \cap \mathbb{Q}$ such that $\lambda(t) \notin\left\{\lambda_{n}: n \in \mathbb{N}\right\}$ for almost every $t \in I$. Additionally, suppose $t \mapsto \lambda(t)$ and $t \mapsto \alpha(t)$ are Lipschitz continuous and $C$ is the right shift operator on $\ell^{p}\left(I_{d}\right) \times \ell^{p}\left(I_{\infty}\right) \cong \ell^{p}\left(I_{\infty}\right)$ :

$$
C\left(z_{1}, \ldots, z_{d}, z_{d+1}, \ldots\right):=\left(0, z_{1}, \ldots, z_{d-1}, z_{d}, \ldots\right) .
$$

Then $t \mapsto A(t)$ is in $W_{*}^{1, \infty}(I, L(X))$ and $t \mapsto A_{0}(t)$ is $\left(M_{0}, 0\right)$-stable (by Lemma 2.17), so that $A$ is $(M, 0)$-stable for some $M \in[1, \infty)$ by Lemma 2.9. Since $\left.A_{0}(t)\right|_{P_{0} X}-\lambda(t)$ is nilpotent of order at most $m_{0}:=\operatorname{dim} \ell^{p}\left(I_{d}\right)=d$ for every $t \in I$ and since $\left.A_{0}(t)\right|_{\left(1-P_{0}\right) X}-$ $\lambda(t)$ is injective and has dense range in $\left(1-P_{0}\right) X$ (because $\lambda(t) \notin\left\{\lambda_{n}: n \in \mathbb{N}\right\}$ ) for almost every $t \in I, P_{0}$ is weakly $m_{0}$-associated with $A_{0}(t)$ and $\lambda(t)$, whence the same is true for $A(t), P$ instead of $A_{0}(t)$ and $P_{0}$. And finally, the resolvent estimate of Theorem 4.3 is clearly fulfilled if we choose $\vartheta(t):=\frac{\pi}{2}$ for all $t \in I$. All other hypotheses of Theorem 4.3 (i) are obvious. 
In the above example, we have chosen $C$ to be the right shift operator on $X=\ell^{p}\left(I_{d}\right) \times$ $\ell^{p}\left(I_{\infty}\right)$ in order to make sure that the example cannot be reduced to a finite-dimensional subspace: there is no finite-dimensional subspace $M$ of $X$ such that

$$
M \supset P(0) X \text { and } A(t) M \subset M \text { as well as } P(t) M \subset M
$$

for every $t \in I$. (Clearly, if given data $A, \lambda, P$ can be reduced to a finite-dimensional subspace $M$ and satisfy the hypotheses of Theorem 4.3 (i), it suffices to prove the respective statement for the reduced data $A^{M}, \lambda, P^{M}$ given by

$$
A^{M}(t):=\left.A(t)\right|_{M} \quad \text { and } \quad P^{M}(t):=\left.P(t)\right|_{M} \quad(t \in I) .
$$

And this, in turn, can typically already be done with the help of the adiabatic theorem with non-uniform spectral gap condition of Section 3: by the finite-dimensionality of $M$, $\lambda(t)$ is isolated in $\sigma\left(A^{M}(t)\right)$ for every $t \in I$ and, by Theorem 2.1 and the first remark following it, $P^{M}(t)$ is the projection associated with $A^{M}(t)$ and $\lambda(t)$ for almost every $t \in I$.) In order to see the claimed irreducibility of the example above, we assume, on the contrary, that $A, \lambda, P$ as given by the example can be reduced to a finite-dimensional subspace $M$ of $X$. Then $R(t) M$ is invariant under $A_{0}(t)$ for every $t \in I$ and hence (by finite-dimensional spectral theory applied to $\left.A_{0}^{\dagger}(t):=\left.A_{0}(t)\right|_{R(t) M}\right)$

$$
R(t) M=\bigoplus_{\lambda \in \sigma_{p}\left(A_{0}^{l}(t)\right)} \bigcup_{k \in \mathbb{N}} \operatorname{ker}\left(A_{0}^{l}(t)-\lambda\right)^{k} \subset \bigoplus_{\lambda \in \sigma_{p}\left(A_{0}^{l}(t)\right)} \bigcup_{k \in \mathbb{N}} \operatorname{ker}\left(A_{0}(t)-\lambda\right)^{k},
$$

which latter space (by the special choice of $A_{0}(t)$ ) for every $t \in I$ contains only vectors with finitely many non-zero components. Consequently, the same is also true for the vectors in $R(t) M$ for every $t \in I$. We now obtain the desired contradiction by observing that the vector $R(t) v=e^{C t} v$, for every $t \neq 0$ and every vector $0 \neq v \in X$ with only finitely many non-zero components, has infinitely many non-zero components because

$$
e^{C t} e_{i}=\left(0, \ldots, 0,1, \frac{t}{1 !}, \frac{t^{2}}{2 !}, \frac{t^{3}}{3 !}, \ldots\right)
$$

for $i \in \mathbb{N}$ (where the entry 1 is in the $i$ th place).

Example 4.7. Suppose $A, \lambda, P$ with $A(t)=R(t)^{-1} A_{0}(t) R(t), P(t)=R(t)^{-1} P_{0} R(t)$, and $R(t)=e^{C t}$ are given as follows in $X:=\ell^{p}\left(I_{d}\right) \times \ell^{p}\left(I_{\infty}\right)$ (where $p \in(1, \infty)$ and $d \in \mathbb{N}):$

$$
A_{0}(t):=\left(\begin{array}{cc}
\lambda(t)+\alpha(t) N & 0 \\
0 & S_{+}-1
\end{array}\right) \quad \text { and } \quad P_{0}:=\left(\begin{array}{ll}
1 & 0 \\
0 & 0
\end{array}\right)
$$

where $\lambda(t) \in \partial U_{1}(-1), \alpha(t), N$ are such that Condition 2.16 is satisfied. Additionally, $t \mapsto \lambda(t)$ and $t \mapsto \alpha(t)$ are Lipschitz continuous and $C$ is the bounded linear map in $\ell^{p}\left(I_{d}\right) \times \ell^{p}\left(I_{\infty}\right) \cong \ell^{p}\left(I_{\infty}\right)$ given by

$$
C\left(z_{1}, \ldots, z_{d}, z_{d+1}, \ldots\right):=\left(0, \ldots, 0, z_{d+1},-z_{d}, 0, \ldots\right),
$$


where in the vector on the right $z_{d+1},-z_{d}$ appear in the $d$ th and $(d+1)$ th place. Since $\lambda(t) \in \partial U_{1}(-1)=\sigma_{c}\left(S_{+}-1\right)$ for every $t \in I$ because $p \neq 1$ (Section 2.4), $P_{0}$ is weakly associated with $A_{0}(t)$ and $\lambda(t)$ and therefore the same goes for $A_{0}(t), P_{0}$ replaced by $A(t)$ and $P(t)$. Also, if for every $t \in I$ we choose $\vartheta(t)$ such that $\lambda(t)=-1+e^{i \vartheta(t)}$, then the resolvent estimate of Theorem 4.3 holds true because

$$
\left\|\left(\lambda(t)+\delta e^{i \vartheta(t)}-A_{0}(t)\right)^{-1}\left(1-P_{0}\right)\right\| \leq\left\|\left(1+\delta-e^{-i \vartheta(t)} S_{+}\right)^{-1}\right\| \leq \frac{1}{\delta}
$$

for every $t \in I$ and $\delta \in(0, \infty)$ (Section 2.4).

Just like the first example, the example above cannot be reduced to a finite-dimensional subspace $M$. Indeed, assuming that $A, \lambda, P$ from above could, we obtain that $R(t) M$ for every $t \in I$ is invariant under $A_{0}(t)$ and

$$
\emptyset \neq \sigma\left(\left.A_{0}(t)\right|_{R(t) M}\right)=\sigma_{p}\left(\left.A_{0}(t)\right|_{R(t) M}\right) \subset \sigma_{p}\left(A_{0}(t)\right)=\{\lambda(t)\}
$$

$\left(\right.$ as $\sigma_{p}\left(S_{+}-1\right)=\emptyset($ Section 2.4)), so that (by finite-dimensional spectral theory)

$$
R(t) M=\operatorname{ker}\left(\left.A_{0}(t)\right|_{R(t) M}-\lambda(t)\right)^{\operatorname{dim} M} \subset \operatorname{ker}\left(A_{0}(t)-\lambda(t)\right)^{\operatorname{dim} M}=P_{0} X \subset M
$$

for all $t \in I$. Consequently, $R(t) P_{0} X \subset R(t) M \subset P_{0} X$. In other words, $P_{0} X$ is invariant under $R(t)=e^{C t}$ for every $t \in I$, which is obviously not true by the choice of $C$ in the above example. Contradiction! We point out that the above example really works only in case $p \neq 1$. In fact, if $p=1$ then $\lambda(t) \in \partial U_{1}(-1)=\sigma_{r}\left(S_{+}-1\right)$ (Section 2.4) from which it follows by the block structure of $A_{0}(t)$ that

$$
\begin{aligned}
& \operatorname{ker}\left(A_{0}(t)-\lambda(t)\right)^{k} \subset \ell^{p}\left(I_{d}\right) \times 0, \\
& \overline{\operatorname{rg}\left(A_{0}(t)-\lambda(t)\right)^{k}} \subset \ell^{p}\left(I_{d}\right) \times \overline{\operatorname{rg}\left(S_{+}-1-\lambda(t)\right)^{k}} \subsetneq \ell^{p}\left(I_{d}\right) \times \ell^{p}\left(I_{\infty}\right)
\end{aligned}
$$

and hence $\operatorname{ker}\left(A_{0}(t)-\lambda(t)\right)^{k}+\overline{\operatorname{rg}\left(A_{0}(t)-\lambda(t)\right)^{k}} \neq X$ for every $k \in \mathbb{N}$ and $t \in I$. Consequently, the adiabatic theorems proven above cannot be applied.

In our last example we show that the conclusion of the adiabatic theorem without spectral gap condition may fail if the regularity hypothesis on $P$ is the only one to be violated.

Example 4.8. Set $A(t):=M_{f_{t}}$ in $X:=L^{p}(\mathbb{R})$ (for some $p \in[1, \infty)$ ), where

$$
f_{t}:=f_{0}(.+t) \quad \text { with } \quad 0 \neq f_{0} \in C_{c}^{1}(\mathbb{R}, i \mathbb{R}),
$$

$\lambda(t):=0$ and $P(t):=M_{\chi_{E_{t}}}$ with $E_{t}:=\left\{f_{t}=0\right\}$. Then all the hypotheses of the adiabatic theorem without spectral gap condition - in the version for projections of infinite rank (second remark after Theorem 4.3) - are fulfilled with the sole exception that $t \mapsto P(t)$ is not SOT-continuously differentiable (by Lemma 2.18). And indeed, the conclusion of the adiabatic theorem already fails: as the $A(t)$ are pairwise commuting and $t \mapsto f_{t}(x)$ is Riemann integrable for every $x \in \mathbb{R}$, one has

$$
\left(U_{\varepsilon}(t, s) g\right)(x)=\left(e^{\frac{1}{\varepsilon} \int_{s}^{t} A(\tau) d \tau} g\right)(x)=e^{\frac{1}{\varepsilon} \int_{s}^{t} f_{\tau}(x) d \tau} g(x)
$$


for almost every $x \in \mathbb{R}$ and therefore $\left(\right.$ by $f_{0}(\mathbb{R}) \subset i \mathbb{R}$ )

$$
\left\|(1-P(t)) U_{\varepsilon}(t) P(0) g\right\|^{p}=\int\left|\left(1-\chi_{E_{t}}(x)\right) \chi_{E_{0}}(x) g(x)\right|^{p} d x
$$

for every $t \in I, \varepsilon \in(0, \infty)$ and $g \in X$. Since the right hand side of this equation does not depend on $\varepsilon \in(0, \infty)$ and since for every $t \in(0,1]$ there is a $g \in X$ such that this right hand side does not vanish, the conclusion of the adiabatic theorem without spectral gap - more precisely, the weaker assertion that $\sup _{t \in I}\left\|(1-P(t)) U_{\varepsilon}(t) P(0) g\right\| \longrightarrow 0$ for all $g \in X-$ fails.

It should be pointed out that the failure of both the hypotheses and the conclusion of the adiabatic theorems without spectral gap condition presented above is a quite typical phenomenon in the case where $A(t)=M_{f_{t}}$ in $X=L^{p}\left(X_{0}\right)$ for some $p \in[1, \infty)$ and some $\sigma$-finite measure space $\left(X_{0}, \mathcal{A}, \mu\right)$. Indeed, if $A(t)=M_{f_{t}}$ in $X=L^{p}\left(X_{0}\right)$ for measurable functions $f_{t}: X_{0} \rightarrow\{\operatorname{Re} z \leq 0\}$ such that $D\left(M_{f_{t}}\right)=D$ for all $t \in I$, if $\lambda(t)$ is an eigenvalue of $A(t)$, and if $P(t)$ for almost every $t \in I$ (with exceptional set $N$ ) is weakly associated with $A(t)$ and $\lambda(t)$, then

$$
P(t)=M_{\chi_{\left\{f_{t}=\lambda(t)\right\}}}=M_{\chi_{E_{t}}} \text { for every } t \in I \backslash N
$$

by Theorem 2.1, and therefore the following holds true. As soon as $I \backslash N \ni t \mapsto P(t)$ is not constant, the hypotheses of the adiabatic theorem without spectral gap (Theorem 4.3) must fail (because then $I \backslash N \ni t \mapsto P(t)=M_{\chi_{E_{t}}}$ cannot extend to an SOT-continuously differentiable map by Lemma 2.18). And as soon as, in addition, the maps $f_{t}$ are $i \mathbb{R}$ valued and $t \mapsto f_{t} g \in X$ is continuous for all $g \in D$, the conclusion of Theorem 4.3, or more precisely, of its corollary

$$
\sup _{t \in I}\left\|(1-P(t)) U_{\varepsilon}(t) P(0)\right\| \longrightarrow 0 \quad \text { and } \sup _{t \in I}\left\|P(t) U_{\varepsilon}(t)(1-P(0))\right\| \longrightarrow 0,
$$

must fail as well. (In order to see this, one gathers from Theorem 2.3 of 41 ] (and its proof) that the evolution system $U_{\varepsilon}$ for $\frac{1}{\varepsilon} A$ exists on $D$ and can be strongly approximated by finite products of operators of the form $e^{M_{f_{\tau}} \sigma}$ with $\tau \in I$ and $\sigma \in[0, \infty)$, and infers from this that for arbitrary $g \in X$

$$
\left|\left(1-\chi_{E_{t}}(x)\right)\left(U_{\varepsilon}(t) \chi_{E_{0}} g\right)(x)-\chi_{E_{t}}(x)\left(U_{\varepsilon}(t)\left(1-\chi_{E_{0}}\right) g\right)(x)\right|=\left|\chi_{E_{t}}(x)-\chi_{E_{0}}(x)\right||g(x)|
$$

for almost every $x \in X_{0}$, whence

$$
\left\|(1-P(t)) U_{\varepsilon}(t) P(0) g-P(t) U_{\varepsilon}(t)(1-P(0)) g\right\|=\|P(t) g-P(0) g\|
$$

for all $t \in I \backslash N, \varepsilon \in(0, \infty)$. Since the right hand side of this equation does not depend on $\varepsilon \in(0, \infty)$ and since $I \backslash N \ni t \mapsto P(t)$ is not constant, there is a $t \in(0,1]$ and a $g \in X$ such that $(1-P(t)) U_{\varepsilon}(t) P(0) g$ and $P(t) U_{\varepsilon}(t)(1-P(0)) g$ do not both converge to 0 as $\varepsilon \searrow 0$.) 


\section{Adiabatic theorems for time-dependent domains}

In this section we extend the adiabatic theorems (with and without spectral gap condition) for time-independent domains of Section 3 and 4 to the case of operators $A(t)$ with time-dependent domains - by slightly modifying the proofs of the respective adiabatic theorems for time-independent domains. Striving for such an extension is very natural because the requirement of constant domains is rather restrictive - just think of differential operators $A(t)$ with (fully) time-dependent boundary conditions. We will see in Section 6 that the adiabatic theorems of this section allow one to almost effortlessly derive adiabatic theorems for operators $A(t)=i A_{a(t)}$ defined by symmetric sesquilinear forms $a(t)$. All the theorems of this section are generalizations of the respective adiabatic theorems for time-independent domains if in these latter theorems all $W_{*}^{n, \infty}$-regulartity requirements are strengthened to $n$ times SOT-continuous differentiability requirements. We will need the following very natural condition on $A$, which takes the role of Condition 2.10 ,

Condition 5.1. $A(t): D(A(t)) \subset X \rightarrow X$ for every $t \in I$ is a densely defined closed linear map such that, for every $\varepsilon \in(0, \infty)$, there is an evolution system $U_{\varepsilon}$ for $\frac{1}{\varepsilon} A$ on $D(A(t))$ and there is a constant $M \in[1, \infty)$ such that $\left\|U_{\varepsilon}(t, s)\right\| \leq M$ for all $(s, t) \in \Delta$ and $\varepsilon \in(0, \infty)$.

We point out that there is a huge number of papers establishing the existence of evolution systems $U$ for a given family $A$ of linear maps $A(t)$ on $D(A(t))$ as, for instance, [27], [34], [49], 28], 221, [4]. See the survey article [48] for many more references. Instead of working with evolution systems on the spaces $Y_{t}=D(A(t))$ as in Condition 5.1, one could also prove adiabatic theorems operating - as in [29] or [30] - with evolution systems for $A$ on certain subspaces $Y$ of the intersection of all $D(A(t))$ (Definition VI.9.2 of [20]), but then one would have to impose various invariance conditions on the subspace $Y$, such as the $A(t)$-admissibiltity of $Y$, the invariance

$$
(z-A(t))^{-1} Y \subset Y
$$

for $z \in \operatorname{rg} \gamma_{t}$ (case with spectral gap) or $z \in\left\{\lambda(t)+\varepsilon e^{i \vartheta(t)}: \varepsilon \in\left(0, \varepsilon_{0}\right]\right\}$ (case without spectral gap), and the invariance of $Y$ under $P(t)$ and $P^{\prime}(t)$. Such invariance conditions, however, are difficult to verify in practice: (5.1), for instance, is clear only for complex numbers $z$ with sufficiently large positive real part (Proposition 2.3 of [29]).

\subsection{Adiabatic theorems with spectral gap condition}

We will need the following condition depending on $m \in\{0\} \cup \mathbb{N} \cup\{\infty\}$ (number of points at which $\sigma($.$) falls into \sigma(A().) \backslash \sigma()$.$) .$

Condition 5.2. $A(t): D(A(t)) \subset X \rightarrow X$ for every $t \in I$ is a linear map such that Condition 5.1 is satisfied. $\sigma(t)$ for every $t \in I$ is a compact subset of $\sigma(A(t)), \sigma($.$) falls$ into $\sigma(A().) \backslash \sigma($.$) at exactly m$ points that accumulate at only finitely many points, and 
$I \backslash N \ni t \mapsto \sigma(t)$ is continuous, where $N$ denotes the set of those $m$ points at which $\sigma($. falls into $\sigma(A().) \backslash \sigma($.$) .$

$$
\begin{gathered}
J_{t_{0}} \ni t \mapsto(z-A(t))^{-1} \text { is SOT-continuously differentiable for all } z \in \operatorname{rg} \gamma_{t_{0}}, \\
\operatorname{rg} \gamma_{t_{0}} \ni z \mapsto \frac{d}{d t}(z-A(t))^{-1} \text { is SOT-continuous for all } t \in J_{t_{0}}, \\
\sup _{(t, z) \in J_{t_{0}} \times \operatorname{rg} \gamma_{t_{0}}}\left\|\frac{d}{d t}(z-A(t))^{-1}\right\|<\infty
\end{gathered}
$$

for every $t_{0} \in I \backslash N$, where the cycle $\gamma_{t_{0}}$ and the non-trivial closed interval $J_{t_{0}} \ni t_{0}$ are chosen such that $\operatorname{rg} \gamma_{t_{0}} \subset \rho(A(t))$ and $\mathrm{n}\left(\gamma_{t_{0}}, \sigma(t)\right)=1$ and $\mathrm{n}\left(\gamma_{t_{0}}, \sigma(A(t)) \backslash \sigma(t)\right)=0$ for every $t \in J_{t_{0}}$. And finally, $P(t)$ is the projection associated with $A(t)$ and $\sigma(t)$ for every $t \in I \backslash N$ and $I \backslash N \ni t \mapsto P(t)$ extends to a twice SOT-continuously differentiable map on the whole of $I$.

With this condition at hand, we can now formulate an adiabatic theorem with uniform spectral gap condition $(m=0)$ and non-uniform spectral gap condition $(m \in \mathbb{N} \cup\{\infty\})$ for time-dependent domains.

Theorem 5.3. Suppose $A(t), \sigma(t), P(t)$ for $t \in I$ are such that Condition 5.2 is satisfied with $m=0$ or $m \in \mathbb{N} \cup\{\infty\}$, respectively. Then

$$
\sup _{t \in I}\left\|U_{\varepsilon}(t)-V_{\varepsilon}(t)\right\|=O(\varepsilon) \text { resp. o(1) } \quad(\varepsilon \searrow 0)
$$

whenever the evolution system $V_{\varepsilon}$ for $\frac{1}{\varepsilon} A+\left[P^{\prime}, P\right]$ exists on $D(A(t))$ for all $\varepsilon \in(0, \infty)$.

Proof. We have only to prove the theorem in the case of a uniform spectral gap $(m=0)$, since the theorem in the case of a non-uniform spectral gap $(m \in \mathbb{N} \cup\{\infty\})$ then follows in the same way as Theorem 3.2 followed from Theorem 3.1. In order to do so, we must only slightly modify the proof of Theorem 3.1. We define the operators $B(t)$ as in the proof of that theorem (where now $\gamma_{t_{0}}$ and $J_{t_{0}}$ are given by Condition 5.2), take over the first preparatory step of that proof, and easily show - instead of what has been shown in the second preparatory step - that $t \mapsto B(t)$ is SOT-continuously differentiable. (It has to be used for this last statement that Condition 5.2 implies

$$
\sup _{(t, z) \in J_{t_{0}} \times \operatorname{rg} \gamma_{t_{0}}}\left\|(z-A(t))^{-1}\right\|<\infty
$$

which can be seen as in the proof of (5.3) below.) We can then almost literally take over the main part of the proof of Theorem 3.1? the only thing that has to be changed is that the $W^{1, \infty}$-regularity of

$$
[0, t] \ni s \mapsto U_{\varepsilon}(t, s) B(s) V_{\varepsilon}(s) x
$$

for $x \in D(A(0))$ can no longer be deduced from Lemma 2.5, but has to be inferred from Lemma 2.6 and Lemma 2.7, and that Proposition 2.13 has to be invoked for an $\varepsilon$-independent bound on $V_{\varepsilon}$. 
In general, the existence of the evolution system $V_{\varepsilon}$ for $\frac{1}{\varepsilon} A+\left[P^{\prime}, P\right]$ on $D(A(t))$ does not seem to be guaranteed under - the fairly general - Condition 5.2. (In view of Proposition 2.13 one would, of course, like to define $V_{\varepsilon}$ as a perturbation series and show that $[s, 1] \ni t \mapsto V_{\varepsilon}(t, s) y$ for every $y \in D(A(s))$ is a continuously differentiable solution to the initial value problem $x^{\prime}=\frac{1}{\varepsilon} A(t) x+\left[P^{\prime}(t), P(t)\right] x, x(s)=y$, but this is not clear in general.) It is therefore good to know that under Condition 5.2 with $m=0$ one has at least the following statement:

$$
\sup _{t \in I}\left\|(1-P(t)) U_{\varepsilon}(t) P(0)\right\|, \quad \sup _{t \in I}\left\|P(t) U_{\varepsilon}(t)(1-P(0))\right\|=O(\varepsilon)
$$

as $\varepsilon \searrow 0$, which follows from the adiabatic theorem of higher order (Theorem 5.7 (i) and (iii) with degree of regularity $n=1$ ) below. It should be pointed out, however, that Theorem 5.3 itself - operating with the evolution systems for $\frac{1}{\varepsilon} A+\left[P^{\prime}, P\right]=\frac{1}{\varepsilon} A_{0 \varepsilon}+K_{0 \varepsilon} \neq$ $\frac{1}{\varepsilon} A_{1 \varepsilon}+K_{1 \varepsilon}$ as comparison evolutions - is not contained in Theorem 5.7

\subsection{Adiabatic theorems without spectral gap condition}

We now prove an adiabatic theorem without spectral gap condition for time-dependent domains in which case we have to explicitly require the differentiability of the resolvent as well as an estimate on the derivative of the resolvent, which two things are no longer automatically satisfied as they were in the case of time-independent domains.

Theorem 5.4. Suppose $A(t): D(A(t)) \subset X \rightarrow X$ for every $t \in I$ is a linear map such that Condition 5.1 is satisfied. Suppose further that $\lambda(t)$ for every $t \in I$ is an eigenvalue of $A(t)$, and that there are numbers $\delta_{0} \in(0, \infty)$ and $\vartheta(t) \in \mathbb{R}$ such that $\lambda(t)+\delta e^{i \vartheta(t)} \in \rho(A(t))$ for all $\delta \in\left(0, \delta_{0}\right]$ and $t \in I$ and such that $t \mapsto \lambda(t)$ and $t \mapsto e^{i \vartheta(t)}$ are continuously differentiable and $t \mapsto\left(\lambda(t)+\delta e^{i \vartheta(t)}-A(t)\right)^{-1}$ is SOT-continuously differentiable. Suppose finally that $P(t)$ for every $t \in I$ is a bounded projection in $X$ commuting with $A(t)$ such that $P(t)$ for almost every $t \in I$ is weakly associated with $A(t)$ and $\lambda(t)$ and that

$$
P(t) X \subset \operatorname{ker}(A(t)-\lambda(t))^{m_{0}}
$$

for every $t \in I$ (and some $m_{0} \in \mathbb{N}$ ). Additionally, suppose that there are $M_{0}, M_{0}^{\prime} \in(0, \infty)$ such that

$$
\begin{aligned}
\left\|\left(\lambda(t)+\delta e^{i \vartheta(t)}-A(t)\right)^{-1}(1-P(t))\right\| & \leq \frac{M_{0}}{\delta}, \\
& \left\|\frac{d}{d t}\left(\left(\lambda(t)+\delta e^{i \vartheta(t)}-A(t)\right)^{-1}(1-P(t))\right)\right\| \leq \frac{M_{0}^{\prime}}{\delta^{m_{0}+1}}
\end{aligned}
$$

for all $\delta \in\left(0, \delta_{0}\right]$ and $t \in I$, let $\operatorname{rk} P(0)<\infty$ and let $t \mapsto P(t)$ be SOT-continuously differentiable. Then

$$
\sup _{t \in I}\left\|\left(U_{\varepsilon}(t)-V_{0 \varepsilon}(t)\right) P(0)\right\| \longrightarrow 0 \quad(\varepsilon \searrow 0),
$$


where $V_{0 \varepsilon}$ for every $\varepsilon \in(0, \infty)$ denotes the evolution system for $\frac{1}{\varepsilon} A P+\left[P^{\prime}, P\right]$ on $X$. If, in addition, $X$ is reflexive and $t \mapsto P(t)$ norm continuously differentiable, then

$$
\sup _{t \in I}\left\|U_{\varepsilon}(t)-V_{\varepsilon}(t)\right\| \longrightarrow 0 \quad(\varepsilon \searrow 0)
$$

whenever the evolution system $V_{\varepsilon}$ for $\frac{1}{\varepsilon} A+\left[P^{\prime}, P\right]$ exists on $D(A(t))$ for every $\varepsilon \in(0, \infty)$.

Proof. Apart from three small changes we can take over the proof of Theorem 4.3 (notice that Lemma 4.1 and Lemma 4.2 also apply in the present case of time-dependent domains). What has to be changed is the following: first, the inclusion (4.3) holds true for every $t \in I$ by assumption, while in Section 4 this was derived by a closedness argument. Second, the fact that

$$
[0, t] \ni s \mapsto U_{\varepsilon}(t, s) B_{n} \delta(s) V_{0 \varepsilon}(s) P(0) x \quad \text { resp. } \quad[0, t] \ni s \mapsto U_{\varepsilon}(t, s) B_{n} \delta(s) V_{\varepsilon}(s) x
$$

is the continuous representative of an element in $W^{1, \infty}([0, t], X)$ for all $x \in X$ resp. all $x \in D(A(0))$ can no longer be deduced from Lemma 2.5 but has to be inferred from Lemma 2.6 and Lemma 2.7 - notice that $s \mapsto B_{n} \boldsymbol{\delta}(s)$ is SOT-continuously differentiable (Lemma 4.2) with $B_{n \boldsymbol{\delta}}(s) X \subset D(A(s))$ for every $s \in I$. And third, the derivative of $s \mapsto \bar{R}_{\varepsilon}(s)$ can no longer be explicitly expressed and estimated as in (4.8) but the respective estimate holds true by assumption.

Similarly, one sees that the variants of the adiabatic theorem without spectral gap condition of Section 4 carry over to the case of time-dependent domains as well, provided their hypotheses are adapted in a similar way as above.

\subsection{An adiabatic theorem of higher order}

In this subsection we extend the adiabatic theorem of higher order of Joye and Pfister from [24] to the case of general operetors $A(t)$ with possibly time-dependent domains mainly for the sake of completeness and in order to make clear the relation to the basic adiabatic theorem with spectral gap (Theorem 5.3). We will use the elegant iterative scheme of 24 which we briefly recall (in a slightly modified form).

Suppose $A(t): D(A(t)) \subset X \rightarrow X$ is a densely defined closed linear map and $\gamma_{t}$ is a cycle in $\mathbb{C}$ for every $t \in J$, where $J$ is a compact interval, and let $\varepsilon \in(0, \infty)$ and $n \in \mathbb{N}$. Then $A_{0 \varepsilon}, P_{0 \varepsilon}, K_{0 \varepsilon}$ are called well-defined w.r.t. $\gamma_{t}(t \in J)$ if and only if $\operatorname{rg} \gamma_{t} \subset \rho\left(A_{0 \varepsilon}(t)\right)$ for all $t \in J$, where $A_{0 \varepsilon}(t):=A(t)$, and $J \ni t \mapsto P_{0 \varepsilon}(t)$ is WOTcontinuously differentiable, where

$$
P_{0 \varepsilon}(t):=\frac{1}{2 \pi i} \int_{\gamma_{t}}\left(z-A_{0 \varepsilon}(t)\right)^{-1} d z
$$

In this case $K_{0 \varepsilon}$ is defined by $K_{0 \varepsilon}(t):=\left[P_{0 \varepsilon}^{\prime}(t), P_{0 \varepsilon}(t)\right]$. And, for general $n \in \mathbb{N}, A_{n \varepsilon}$, $P_{n \varepsilon}, K_{n \varepsilon}$ are called well-defined w.r.t. $\gamma_{t}(t \in J)$ if and only if $A_{n-1 \varepsilon}, P_{n-1 \varepsilon}, K_{n-1 \varepsilon}$ 
are well-defined w.r.t. $\gamma_{t}(t \in J), \operatorname{rg} \gamma_{t} \subset \rho\left(A_{n \varepsilon}(t)\right)$ for all $t \in J$, where $A_{n \varepsilon}(t):=$ $A(t)-\varepsilon K_{n-1 \varepsilon}(t)$, and $J \ni t \mapsto P_{n \varepsilon}(t)$ is WOT-continuously differentiable, where

$$
P_{n \varepsilon}(t):=\frac{1}{2 \pi i} \int_{\gamma_{t}}\left(z-A_{n \varepsilon}(t)\right)^{-1} d z .
$$

In this case $K_{n \varepsilon}$ is defined by $K_{n \varepsilon}(t):=\left[P_{n \varepsilon}^{\prime}(t), P_{n \varepsilon}(t)\right]$.

We will need the following conditions depending on $n \in \mathbb{N} \cup\{\infty\}$ (degree of regularity) in the adiabatic theorem of higher order below.

Condition 5.5. $A(t): D(A(t)) \subset X \rightarrow X$ for every $t \in I$ is a densely defined closed linear map. $\sigma(t)$ for every $t \in I$ is a compact and isolated subset of $\sigma(A(t))$, there is an $r_{0}>0$ such that $U_{r_{0}}(\sigma(t)) \backslash \sigma(t) \subset \rho(A(t))$ for all $t \in I$, and $t \mapsto \sigma(t)$ is continuous. For every $t_{0} \in I$, there are positive constants $a_{t_{0}}, b_{t_{0}}, c_{t_{0}}$ such that

$$
\begin{gathered}
J_{t_{0}} \ni t \mapsto(z-A(t))^{-1} \text { is } n \text { times WOT-continuously differentiable for all } z \in \operatorname{rg} \gamma_{t_{0}}, \\
\operatorname{rg} \gamma_{t_{0}} \ni z \mapsto \frac{d^{l}}{d t^{l}}(z-A(t))^{-1} \text { is SOT-continuous for all } t \in J_{t_{0}}, l \in\{1, \ldots, n\}, \\
\sup _{(t, z) \in J_{t_{0}} \times \operatorname{rg} \gamma_{t_{0}}}\left\|\frac{d^{l}}{d t^{l}}(z-A(t))^{-1}\right\| \leq a_{t_{0}} c_{t_{0}}^{l} \frac{l !}{(1+l)^{2}} \text { for all } l \in\{1, \ldots, n\},
\end{gathered}
$$

where $\gamma_{t_{0}}$ is a cycle in $\bar{U}_{\frac{4 r_{0}}{7}}\left(\sigma\left(t_{0}\right)\right) \backslash U_{\frac{3 r_{0}}{7}}\left(\sigma\left(t_{0}\right)\right)$ with

$$
\mathrm{n}\left(\gamma_{t_{0}}, U_{\frac{3 r_{0}}{7}}\left(\sigma\left(t_{0}\right)\right)\right)=1 \quad \text { and } \quad \mathrm{n}\left(\gamma_{t_{0}}, \mathbb{C} \backslash \bar{U}_{\frac{4 r_{0}}{7}}\left(\sigma\left(t_{0}\right)\right)\right)=0
$$

and where $J_{t_{0}} \subset I$ is a non-trivial closed interval containing $t_{0}$ such that $\sigma(t) \subset U_{\frac{r_{0}}{7}}\left(\sigma\left(t_{0}\right)\right)$ and $\sigma\left(t_{0}\right) \subset U_{\frac{r_{0}}{7}}(\sigma(t))$ for all $t \in J_{t_{0}}$. And finally, $P(t)$ for every $t \in I$ is the projection associated with $A(t)$ and $\sigma(t), t \mapsto P(t)$ is $n+1$ times WOT-continuously differentiable and

$$
\sup _{t \in J_{t_{0}}}\left\|\frac{d^{l}}{d t^{l}}\left[P^{\prime}(t), P(t)\right]\right\| \leq b_{t_{0}} c_{t_{0}}^{l} \frac{l !}{(1+l)^{2}} \text { for all } l \in\{0,1, \ldots, n\} \text { and } t_{0} \in I .
$$

In the special case of time-independent domains $D(A(t))=D$, one easily sees - using the remark before Lemma 2.5 - that the requirements on the resolvent of $A(t)$ in Condition 5.5 are fulfilled for an $n \in \mathbb{N}$ if, for instance, $t \mapsto A(t) x$ is $n$ times weakly continuously differentiable for all $x \in D$. And they are fulfilled for $n=\infty$ if, for instance, there is an open neighbourhood $U_{I}$ of $I$ in $\mathbb{C}$ such that, for every $x \in D, t \mapsto A(t) x$ extends to a holomorphic map on $U_{I}$ (Cauchy inequalities!).

Lemma 5.6 (Joye-Pfister). (i) Suppose that Condition 5.5 is satisfied for an $n \in \mathbb{N}$. Then there is an $\varepsilon^{*}>0$ such that $A_{n \varepsilon}, P_{n \varepsilon}, K_{n \varepsilon}$ are well-defined w.r.t. $\gamma_{t}(t \in I)$ for every $\varepsilon \in\left(0, \varepsilon^{*}\right]$. Furthermore,

$$
\sup _{t \in I}\left\|K_{n \varepsilon}(t)-K_{n-1 \varepsilon}(t)\right\|=O\left(\varepsilon^{n}\right) \quad(\varepsilon \searrow 0) .
$$


(ii) Suppose that Condition 5.5 is satisfied for $n=\infty$. Then there is an $\varepsilon^{*}>0$ and for every $\varepsilon \in\left(0, \varepsilon^{*}\right]$ there is a natural number $n^{*}(\varepsilon) \in \mathbb{N}$ such that $A_{n^{*}(\varepsilon) \varepsilon}, P_{n^{*}(\varepsilon) \varepsilon}, K_{n^{*}(\varepsilon) \varepsilon}$ are well-defined w.r.t. $\gamma_{t}(t \in I)$ for every $\varepsilon \in\left(0, \varepsilon^{*}\right]$. Furthermore, there is a constant $g \in(0, \infty)$ such that

$$
\sup _{t \in I}\left\|K_{n^{*}(\varepsilon) \varepsilon}(t)-K_{n^{*}(\varepsilon)-1 \varepsilon}(t)\right\|=O\left(e^{-\frac{g}{\varepsilon}}\right) \quad(\varepsilon \searrow 0) .
$$

Proof. We begin with some general preparatory considerations from which both part (i) and part (ii) will easily follow. Suppose (for the entire proof) that Condition 5.5 is satisfied for $n=1$ and fix $t_{0} \in I$ for the moment. We have

$$
J_{t_{0}} \times C_{t_{0}}:=J_{t_{0}} \times \bar{U}_{\frac{5 r_{0}}{7}}\left(\sigma\left(t_{0}\right)\right) \backslash U_{\frac{2 r_{0}}{7}}\left(\sigma\left(t_{0}\right)\right) \subset \subset\left\{(t, z) \in J_{t_{0}} \times \mathbb{C}: z \in \rho(A(t))\right\}=: U_{t_{0}}
$$

and $U_{t_{0}} \ni(t, z) \mapsto(z-A(t))^{-1}$ is continuous, because $J_{t_{0}} \ni t \mapsto A(t)$ is continuous in the generalized sense due to the WOT-continuous differentiability of $J_{t_{0}} \ni t \mapsto(z-A(t))^{-1}$ (Theorem IV.3.15 of [31]). Consequently, $\sup _{(t, z) \in J_{t_{0}} \times C_{t_{0}}}\left\|(z-A(t))^{-1}\right\|<\infty$, whence we can (and will) assume w.l.o.g. that

$$
\sup _{(t, z) \in J_{t_{0}} \times C_{t_{0}}}\left\|(z-A(t))^{-1}\right\| \leq a_{t_{0}} .
$$

We now define $\varepsilon_{t_{0}}^{*}$ and $n_{t_{0}}^{*}(\varepsilon)$ just like in Joye and Pfister's paper [24], that is,

$$
\begin{gathered}
\varepsilon_{t_{0}}^{*}:=\max \left\{\varepsilon \in\left(0, \frac{1}{2 a_{t_{0}} b_{t_{0}}}\right): \sum_{k=1}^{\infty}\left(2 \alpha^{2} a_{t_{0}} b_{t_{0}} \frac{\varepsilon}{1-2 a_{t_{0}} b_{t_{0}} \varepsilon}\right)^{k} \leq \alpha\right\}, \\
n_{t_{0}}^{*}(\varepsilon):=\left\lfloor\frac{1}{e c_{t_{0}} d_{t_{0}} \varepsilon}\right\rfloor \text { for } \varepsilon \in(0, \infty),
\end{gathered}
$$

where $\alpha$ and $d_{t_{0}}$ are defined by equation (2.30) and equation (2.50) of [24]. (In particular, $\varepsilon_{t_{0}}^{*}$ and $n_{t_{0}}^{*}(\varepsilon)$ only depend on $\gamma_{t_{0}}, a_{t_{0}}, b_{t_{0}}$ and $c_{t_{0}}$.) We now show by finite induction over $k$ : whenever Condition 5.5 is satisfied for a certain $n^{\prime} \in \mathbb{N}$, then the following holds true for all $\varepsilon \in\left(0, \varepsilon_{t_{0}}^{*}\right]$ and all $k \in\left\{1, \ldots, n_{t_{0}}^{*}\left(\varepsilon, n^{\prime}\right)\right\}$ with $n_{t_{0}}^{*}\left(\varepsilon, n^{\prime}\right):=\min \left\{n_{t_{0}}^{*}(\varepsilon), n^{\prime}\right\}$ :

(a) $A_{k \varepsilon}, P_{k \varepsilon}, K_{k \varepsilon}$ are well-defined w.r.t. $\gamma_{t}\left(t \in J_{t_{0}}\right)$ and $J_{t_{0}} \ni t \mapsto K_{k \varepsilon}(t)$ is $n_{t_{0}}^{*}\left(\varepsilon, n^{\prime}\right)-$ $k$ times WOT-continuously differentiable

(b) $\sup _{t \in J_{t_{0}}}\left\|K_{k \varepsilon}^{(l)}(t)-K_{k-1 \varepsilon}^{(l)}(t)\right\| \leq b_{t_{0}} c_{t_{0}}^{k+l} d_{t_{0}}^{k} \varepsilon^{k} \frac{(k+l) !}{(1+l)^{2}}$ for all $l \in \mathbb{N} \cup\{0\}$ with the property that $k+l \leq n_{t_{0}}^{*}\left(\varepsilon, n^{\prime}\right)$

(c) $\sup _{t \in J_{t_{0}}}\left\|K_{k \varepsilon}^{(l)}(t)\right\| \leq 2 b_{t_{0}} c_{t_{0}}^{l} \frac{l !}{(1+l)^{2}}$ for all $l \in \mathbb{N} \cup\{0\}$ with $k+l \leq n_{t_{0}}^{*}\left(\varepsilon, n^{\prime}\right)$.

Suppose that Condition 5.5 is satisfied for a certain $n^{\prime} \in \mathbb{N}$ and fix $\varepsilon \in\left(0, \varepsilon_{t_{0}}^{*}\right.$. Set $k=1$ for the induction basis. We have only to prove assertion (a) since assertions (b) and (c) can be gathered from the proof of Proposition 2.1 of [24]. It is obvious that $A_{0 \varepsilon}, P_{0 \varepsilon}$, 
$K_{0 \varepsilon}$ are well-defined w.r.t. $\gamma_{t}\left(t \in J_{t_{0}}\right)$ and that $t \mapsto K_{0 \varepsilon}(t)=\left[P^{\prime}(t), P(t)\right]$ is $n^{\prime}$ times WOT-continuously differentiable. Since, for $z \in C_{t_{0}}$ and $t \in J_{t_{0}}$,

$$
\begin{gathered}
\left(z-A_{1 \varepsilon}(t)\right)=\left(1+\varepsilon K_{0 \varepsilon}(t)(z-A(t))^{-1}\right)(z-A(t)) \\
\text { and }\left\|\varepsilon K_{0 \varepsilon}(t)(z-A(t))^{-1}\right\| \leq \varepsilon b_{t_{0}}\left\|(z-A(t))^{-1}\right\| \leq \varepsilon_{t_{0}}^{*} b_{t_{0}} a_{t_{0}}<\frac{1}{2}
\end{gathered}
$$

(remember the estimate for $K_{0 \varepsilon}=\left[P^{\prime}, P\right]$ from Condition [5.5, the estimate for the resolvent of $A$ from (5.3), and the definition of $\varepsilon_{t_{0}}^{*}$ in (5.4) $)$, we see that

$$
\operatorname{rg} \gamma_{t} \subset \bar{U}_{\frac{4 r_{0}}{7}}(\sigma(t)) \backslash U_{\frac{3 r_{0}}{7}}(\sigma(t)) \subset \bar{U}_{\frac{5 r_{0}}{7}}\left(\sigma\left(t_{0}\right)\right) \backslash U_{\frac{2 r_{0}}{7}}\left(\sigma\left(t_{0}\right)\right)=C_{t_{0}} \subset \rho\left(A_{1 \varepsilon}(t)\right)
$$

for all $t \in J_{t_{0}}$. And since

$$
\begin{gathered}
\mathrm{n}\left(\gamma_{t}, U_{\frac{2 r_{0}}{7}}\left(\sigma\left(t_{0}\right)\right)\right)=1=\mathrm{n}\left(\gamma_{t_{0}}, U_{\frac{2 r_{0}}{7}}\left(\sigma\left(t_{0}\right)\right)\right), \\
\mathrm{n}\left(\gamma_{t}, \mathbb{C} \backslash \bar{U}_{\frac{5 r_{0}}{7}}\left(\sigma\left(t_{0}\right)\right)\right)=0=\mathrm{n}\left(\gamma_{t_{0}}, \mathbb{C} \backslash \bar{U}_{\frac{5 r_{0}}{7}}\left(\sigma\left(t_{0}\right)\right)\right)
\end{gathered}
$$

and $C_{t_{0}} \subset \rho\left(A_{1 \varepsilon}(t)\right)$ for all $t \in J_{t_{0}}$, the cycles $\gamma_{t}$ and $\gamma_{t_{0}}$ are homologous in $\rho\left(A_{1 \varepsilon}(t)\right)$ for $t \in J_{t_{0}}$, so that

$$
\begin{aligned}
J_{t_{0}} \ni t \mapsto P_{1 \varepsilon}(t) & =\frac{1}{2 \pi i} \int_{\gamma_{t}}\left(z-A_{1 \varepsilon}(t)\right)^{-1} d z \\
& =\frac{1}{2 \pi i} \int_{\gamma_{t_{0}}}(z-A(t))^{-1}\left(1+\varepsilon K_{0 \varepsilon}(t)(z-A(t))^{-1}\right)^{-1} d z
\end{aligned}
$$

is $n^{\prime}$ times WOT-continuously differentiable. (In order to see this, use the product rule and inverses rule for WOT-continuous differentiability from the remark before Lemma 2.5 as well as Condition 5.5) Consequently, $A_{1 \varepsilon}, P_{1 \varepsilon}, K_{1 \varepsilon}$ are well-defined w.r.t. $\gamma_{t}\left(t \in J_{t_{0}}\right)$ and $t \mapsto K_{1 \varepsilon}(t)$ is $n^{\prime}-1$ times (in particular, $n_{t_{0}}^{*}\left(\varepsilon, n^{\prime}\right)-1$ times) WOT-continuously differentiable.

Choose now $k \in\left\{2, \ldots, n_{t_{0}}^{*}\left(\varepsilon, n^{\prime}\right)\right\}$ and assume that assertions (a), (b), (c) are true for $k-1$. We then have to show that they are also true for $k$. As above we have only to establish (a) since (b) and (c) can then be derived as in the proof of Proposition 2.1 of [24], as a close inspection of that proof shows. And in order to prove (a) we can proceed essentially as above: just use assertion (c) for $k-1$ to get the estimate

$$
\sup _{(t, z) \in J_{t_{0}} \times C_{t_{0}}}\left\|\varepsilon K_{k-1 \varepsilon}(t)(z-A(t))^{-1}\right\| \leq 2 b_{t_{0}} a_{t_{0}} \varepsilon_{t_{0}}^{*}<1
$$

and continue as above, thereby concluding the inductive proof of (a), (b), (c).

Choosing finitely many points $t_{1}, \ldots, t_{m} \in I$ such that $J_{t_{1}} \cup \cdots \cup J_{t_{m}}=I$, and setting

$$
\varepsilon^{*}:=\min \left\{\varepsilon_{t_{1}}^{*}, \ldots, \varepsilon_{t_{m}}^{*}\right\} \text { and } n^{*}(\varepsilon):=\min \left\{n_{t_{1}}^{*}(\varepsilon), \ldots, n_{t_{m}}^{*}(\varepsilon)\right\},
$$


we find - in virtue of the above preparations - that, for every $\varepsilon \in\left(0, \varepsilon^{*}\right]$, the following holds true: whenever Condition [5.5 is fulfilled for an $n^{\prime} \in \mathbb{N}$, then $A_{k \varepsilon}, P_{k \varepsilon}, K_{k \varepsilon}$ are well-defined w.r.t. $\gamma_{t}(t \in I)$ and

$$
\sup _{t \in I}\left\|K_{k \varepsilon}(t)-K_{k-1 \varepsilon}(t)\right\| \leq b c^{k} d^{k} \varepsilon^{k} k !
$$

for every $k \in\left\{1, \ldots, n^{*}\left(\varepsilon, n^{\prime}\right)\right\}$, where $b, c, d$ are obtained by taking the maximum of the corresponding quantities for the points $t_{1}, \ldots, t_{m}$ and $n^{*}\left(\varepsilon, n^{\prime}\right):=\min \left\{n^{*}(\varepsilon), n^{\prime}\right\}$.

Suppose now as in (i) that Condition 5.5 is satisfied for an $n \in \mathbb{N}$. Since $n^{*}(\varepsilon) \longrightarrow \infty$ as $\varepsilon \searrow 0$, we can assume w.l.o.g. that $n^{*}(\varepsilon, n)=n$ for all $\varepsilon \in\left(0, \varepsilon^{*}\right]$ and therefore assertion (i) follows from (5.6). Suppose finally as in (ii) that Condition 5.5 is satisfied for $n=\infty$. Since for every $\varepsilon \in\left(0, \varepsilon^{*}\right]$ there is $n^{\prime} \in \mathbb{N}$ such that $n^{*}\left(\varepsilon, n^{\prime}\right)=n^{*}(\varepsilon)$ and since Condition 5.5 is satisfied, in particular, for this $n^{\prime}$, assertion (ii) follows from (5.6) with the help of Stirling's formula (see, for instance, the proof of Theorem 2.1 of [24] or of Theorem $1 \mathrm{~b}$ of [40]).

After these preparations we can now prove the announced adiabatic theorem of higher order extending Theorem 2.1 of [24] where skew self-adjoint operators $A(t)$ that analytically depend on $t$ and have time-independent domains are considered.

Theorem 5.7. Suppose $A(t), \sigma(t), P(t)$ for $t \in I$ are such that Condition 5.1 is satisfied and Condition 5.5 with WOT replaced by SOT is satisfied for an $n \in \mathbb{N}$ or $n=\infty$, respectively. Then

(i) $\sup _{t \in I}\left\|P_{\varepsilon}(t)-P(t)\right\|=O(\varepsilon)$ as $\varepsilon \searrow 0$, where, for all $\varepsilon \in\left(0, \varepsilon^{*}\right]$ and $t \in I$, $P_{\varepsilon}(t):=P_{n \varepsilon}(t)$ in case $n \in \mathbb{N}$ and $P_{\varepsilon}(t):=P_{n^{*}(\varepsilon) \varepsilon}(t)$ in case $n=\infty$ (and where $\varepsilon^{*}$ and $n^{*}(\varepsilon)$ are defined as in (5.5) of the lemma above).

(ii) Whenever the evolution system $V_{\varepsilon}$ for $\frac{1}{\varepsilon} A_{n \varepsilon}+K_{n \varepsilon}$ resp. $\frac{1}{\varepsilon} A_{n^{*}(\varepsilon) \varepsilon}+K_{n^{*}(\varepsilon) \varepsilon}$ exists on $D(A(t))$ for all $\varepsilon \in\left(0, \varepsilon^{*}\right]$, then $V_{\varepsilon}$ is adiabatic w.r.t. $P_{\varepsilon}$ and for a suitable constant $g \in(0, \infty)$

$$
\sup _{t \in I}\left\|V_{\varepsilon}(t)-U_{\varepsilon}(t)\right\|=O\left(\varepsilon^{n}\right) \text { resp. } O\left(e^{-\frac{g}{\varepsilon}}\right) \quad(\varepsilon \searrow 0) .
$$

(iii) Additionally, one has - the existence of $V_{\frac{1}{\varepsilon}}$ being irrelevant here - that

$$
\begin{aligned}
\sup _{t \in I} \|(1- & \left.P_{\varepsilon}(t)\right) U_{\varepsilon}(t) P_{\varepsilon}(0) \|, \\
& \sup _{t \in I}\left\|P_{\varepsilon}(t) U_{\varepsilon}(t)\left(1-P_{\varepsilon}(0)\right)\right\|=O\left(\varepsilon^{n}\right) \text { resp. } O\left(e^{-\frac{g}{\varepsilon}}\right) \quad(\varepsilon \searrow 0) .
\end{aligned}
$$

Proof. (i) Set $A_{\varepsilon}(t):=A_{n \varepsilon}(t)$ and $K_{\varepsilon}^{-}(t):=K_{n-1 \varepsilon}(t)$ in case $n \in \mathbb{N}$ and $A_{\varepsilon}(t):=$ $A_{n^{*}(\varepsilon) \varepsilon}(t)$ and $K_{\varepsilon}^{-}(t):=K_{n^{*}(\varepsilon)-1 \varepsilon}(t)$ in case $n=\infty$ (for $t \in I$ and $\left.\varepsilon \in\left(0, \varepsilon^{*}\right]\right)$. As was shown in the proof of the above lemma, the cycles $\gamma_{t}$ and $\gamma_{t_{i}}$ are homologous in $\rho\left(A_{\varepsilon}(t)\right)$ 
for every $t \in J_{t_{i}}$ (where $t_{1}, \ldots, t_{m}$ are points of $I$ chosen as in the definition of $\varepsilon^{*}$ and $n^{*}(\varepsilon)$ in (5.5) ) and every $\varepsilon \in\left(0, \varepsilon^{*}\right]$, whence

$$
\begin{aligned}
P_{\varepsilon}(t)-P(t) & =\frac{1}{2 \pi i} \int_{\gamma_{t_{i}}}\left(z-A_{\varepsilon}(t)\right)^{-1}-(z-A(t))^{-1} d z \\
& =-\frac{1}{2 \pi i} \int_{\gamma_{t_{i}}}\left(z-A_{\varepsilon}(t)\right)^{-1} \varepsilon K_{\varepsilon}^{-}(t)(z-A(t))^{-1} d z
\end{aligned}
$$

for all $t \in J_{t_{i}}$ and $\varepsilon \in\left(0, \varepsilon^{*}\right]$. Also, it was shown in the proof of the above lemma that for all $\varepsilon \in\left(0, \varepsilon^{*}\right]$ and all $i \in\{1, \ldots, m\}$ one has $\sup _{(t, z) \in J_{t_{i}} \times \operatorname{rg} \gamma_{t_{i}}}\left\|(z-A(t))^{-1}\right\| \leq a_{t_{i}}$, $\sup _{t \in J_{t_{i}}}\left\|K_{\varepsilon}^{-}(t)\right\| \leq 2 b_{t_{i}}$, and

$$
\begin{aligned}
\left\|\left(z-A_{\varepsilon}(t)\right)^{-1}\right\| & \leq\left\|(z-A(t))^{-1}\right\|\left\|\left(1+\varepsilon K_{\varepsilon}^{-}(t)(z-A(t))^{-1}\right)^{-1}\right\| \\
& \leq a_{t_{i}} \sum_{m=0}^{\infty}\left(\varepsilon 2 b_{t_{i}} a_{t_{i}}\right)^{m} \leq \frac{a_{t_{i}}}{1-2 a_{t_{i}} b_{t_{i}} \varepsilon_{t_{i}}^{*}}<\infty
\end{aligned}
$$

for all $(t, z) \in J_{t_{i}} \times \operatorname{rg} \gamma_{t_{i}}$. Assertion (i) is now clear (notice that for this assertion Condition 5.1 is not needed - only Condition 5.5 in its original WOT version is used).

(ii) Set $K_{\varepsilon}^{+}(t):=\left[P_{\varepsilon}^{\prime}(t), P_{\varepsilon}(t)\right]$ for $t \in I$ and $\varepsilon \in\left(0, \varepsilon^{*}\right]$ and suppose that the evolution system $V_{\varepsilon}$ for $\frac{1}{\varepsilon} A_{\varepsilon}+K_{\varepsilon}^{+}$exists on $D(A(t))$. Since for every $x \in D(A(0))$ the map $[0, t] \ni s \mapsto U_{\varepsilon}(t, s) V_{\varepsilon}(s) x$ is continuous and right differentiable (by Lemma 2.6) and since the right derivative $s \mapsto U_{\varepsilon}(t, s)\left(K_{\varepsilon}^{+}(s)-K_{\varepsilon}^{-}(s)\right) V_{\varepsilon}(s) x$ is bounded, it follows from Lemma 2.7 that

$$
\begin{aligned}
V_{\varepsilon}(t) x-U_{\varepsilon}(t) x & =\left.U_{\varepsilon}(t, s) V_{\varepsilon}(s) x\right|_{s=0} ^{s=t} \\
& =\int_{0}^{t} U_{\varepsilon}(t, s)\left(K_{\varepsilon}^{+}(s)-K_{\varepsilon}^{-}(s)\right) V_{\varepsilon}(s) x d s
\end{aligned}
$$

for all $t \in I$. And from this, in turn, we conclude the desired estimates - using the estimates for $K_{\varepsilon}^{+}-K_{\varepsilon}^{-}$from Lemma 5.6 and applying Proposition 2.13 (ii). It remains to show that $V_{\varepsilon}$ is adiabatic w.r.t. $P_{\varepsilon}$. As, by assumption, Condition 5.5 is satisfied with WOT replaced by SOT (up to now, the unaltered Condition 5.5 was sufficient), $t \mapsto P_{\varepsilon}(t)$ is continuously differentiable not only w.r.t. WOT but also w.r.t. SOT. And therefore, the adiabaticity of $V_{\varepsilon}$ follows from Propostion 2.14.

(iii) Arguing as in the adiabaticity proof above, we get for every $x \in D(A(0))$ and every $t \in I$ that

$$
\begin{aligned}
P_{\varepsilon}(t) U_{\varepsilon}(t) x-U_{\varepsilon}(t) P_{\varepsilon}(0) x & =\left.U_{\varepsilon}(t, s) P_{\varepsilon}(s) U_{\varepsilon}(s) x\right|_{s=0} ^{s=t} \\
& =\int_{0}^{t} U_{\varepsilon}(t, s)\left(P_{\varepsilon}^{\prime}(s)-\frac{1}{\varepsilon}\left(A(s) P_{\varepsilon}(s)-P_{\varepsilon}(s) A(s)\right)\right) U_{\varepsilon}(s) x d s .
\end{aligned}
$$

Since $A_{\varepsilon}(s)$ commutes with $P_{\varepsilon}(s)$ for $s \in I$ and since $A=A_{\varepsilon}+\varepsilon K_{\varepsilon}^{-}$, we have

$$
\begin{aligned}
P_{\varepsilon}^{\prime}(s) & -\frac{1}{\varepsilon}\left(A(s) P_{\varepsilon}(s)-P_{\varepsilon}(s) A(s)\right) \subset P_{\varepsilon}^{\prime}(s)-\left[K_{\varepsilon}^{-}(s), P_{\varepsilon}(s)\right] \\
& =P_{\varepsilon}^{\prime}(s)-\left[K_{\varepsilon}^{+}(s), P_{\varepsilon}(s)\right]+\left[K_{\varepsilon}^{+}(s)-K_{\varepsilon}^{-}(s), P_{\varepsilon}(s)\right]=\left[K_{\varepsilon}^{+}(s)-K_{\varepsilon}^{-}(s), P_{\varepsilon}(s)\right]
\end{aligned}
$$


for every $s \in I$, and the desired conclusion follows with the help of Lemma 5.6.

It is obvious from the definition of Joye and Pfister's iterative scheme that $P_{\varepsilon}(t)=$ $P(t)$ for all $t$ in the (possibly empty) set $I \backslash \operatorname{supp} P^{\prime}$, and therefore it follows from Theorem 5.7 (iii) that

$$
\begin{aligned}
\sup _{t \in I \backslash \operatorname{supp} P^{\prime}} \| & \left\|(1-P(t)) U_{\frac{1}{\varepsilon}}(t) P(0)\right\|, \\
& \sup _{t \in I \backslash \operatorname{supp} P^{\prime}}\left\|P(t) U_{\frac{1}{\varepsilon}}(t)(1-P(0))\right\|=O\left(\varepsilon^{n}\right) \text { resp. } O\left(e^{-\frac{g}{\varepsilon}}\right) \quad(\varepsilon \searrow 0) .
\end{aligned}
$$

A result similar to Theorem 5.7 could have been proved with the help of a method developed by Nenciu in [40] - this can easily be gathered from the exposition in Section 7 of [46]. We have chosen Joye and Pfister's method since it is easier to remember and effortlessly transferred to the case of several compact isolated subsets $\sigma_{1}(t), \ldots, \sigma_{m}(t)$ of $\sigma(A(t))$ where each is uniformly isolated in $\sigma(A(t))$ and uniformly isolated from each of the others.

We finally comment on a recent theorem by Joye from [25] dealing with time-independent domains and several spectral subsets $\sigma_{i}(t)$. It allows for a generalization of Condition 5.1 at the cost of a specialization of Condition 5.5 and states the following (where we confine ourselves, for the sake of notational simplicity, to the case of only one spectral subset $\left.\sigma_{i}(t)=\sigma(t)\right)$ : if - and what follows is a special case of Condition 5.5 - there is an open neighbourhood $U_{I}$ of $I$ such that $t \mapsto A(t) x$ for every $x \in D$ extends to a holomorphic map on $U_{I}$ and if $\sigma(t)=\{\lambda(t)\}$ for every $t \in I$ for a uniformly isolated spectral value $\lambda(t)$ of $A(t)$ of finite algebraic multiplicity (hence an eigenvalue) such that $t \mapsto \lambda(t)$ is continuous, then it suffices for the conclusion of Theorem 5.7 to hold that - instead of Condition 5.1- $-\lambda(t)$ lie in the left closed complex half-plane and $A(t) \bar{P}(t)$ generate a contraction semigroup on $X$ for every $t \in I$ (where $\bar{P}:=1-P$ ). So, in the above-mentioned special case of Condition [5.5 the boundedness requirement on $U_{\varepsilon}$ from Condition 5.1 is not necessary for assertions (i), (ii) and (iii) of Theorem [5.7. It is, however, necessary for the convergences

$$
\sup _{t \in I}\left\|(1-P(t)) U_{\varepsilon}(t) P(0)\right\|, \quad \sup _{t \in I}\left\|P(t) U_{\varepsilon}(t)(1-P(0))\right\| \longrightarrow 0 \quad(\varepsilon \searrow 0)
$$

with the originally given projections $P(t)$, which we are primarily interested in here. See the example at the end of Section 1 of [25] for a proof of this necessity statement. Also, it should be remarked that the above-mentioned special requirements (analyticity and finite algebraic multiplicity) of Joye's theorem from [25] are really essential for the proof in [25]. Indeed, this proof essentially rests upon the following estimate for the evolution system $V_{0 \varepsilon}$ for $\frac{1}{\varepsilon} A_{0 \varepsilon}+K_{0 \varepsilon}=\frac{1}{\varepsilon} A+\left[P^{\prime}, P\right]$ on $D$

$$
\sup _{(s, t) \in \Delta}\left\|V_{0 \varepsilon}(t, s)\right\| \leq c e^{c / \varepsilon^{\beta}} \quad\left(\varepsilon \in\left(0, \varepsilon^{*}\right]\right)
$$


with constants $\beta \in(0,1)$ and $c \in(0, \infty)$ (Proposition 6.1 of [25]), which then - by the usual perturbation argument (Proposition 2.13) - yields the estimates

$$
\sup _{(s, t) \in \Delta}\left\|U_{\varepsilon}(t, s)\right\|, \sup _{(s, t) \in \Delta}\left\|V_{\varepsilon}(t, s)\right\| \leq c^{\prime} e^{c^{\prime} / \varepsilon^{\beta}} \quad\left(\varepsilon \in\left(0, \varepsilon^{*}\right]\right)
$$

from which, in turn, by the integral representation (5.7) and the exponential decay of $K_{\varepsilon}^{+}-K_{\varepsilon}^{-}$from Lemma 5.6 (analyticity requirement!), the conclusion of Theorem 5.7 finally follows. And the fundamental estimate (5.8) rests upon a result on the growth (in $\varepsilon)$ of the evolution system for analytic families $\frac{1}{\varepsilon} N$ of nilpotent operators $N(t)$ on finitedimensional spaces (Proposition 4.1 of [25]), which proposition (by the analyticity and finite algebraic multiplicity requirement!) can be applied to the nilpotent endomorphisms

$$
N(t):=\left.W(t)^{-1}(A(t)-\lambda(t)) W(t)\right|_{P(0) X}
$$

of the finite-dimensional space $P(0) X$ that analytically depend on $t$. $W$ denotes the evolution system for $\left[P^{\prime}, P\right]$ on $X$ exactly intertwining the subspaces $P(t) X$.

\subsection{An example with time-dependent domains}

We confine ourselves to an example illustrating the adiabatic theorem without spectral gap condition. In this example, a differential operator of the simplest kind occurs, namely $B: W^{1, p}(\mathbb{R}) \subset L^{p}(\mathbb{R}) \rightarrow L^{p}(\mathbb{R})$ with $B f:=\partial f$ (weak derivative). Since $B$ is the generator of the (left) translation group $T$ on $L^{p}(\mathbb{R})$ (which is given by $T(t) f:=f(.+t)$ for $t \in \mathbb{R}$ ), one has $\sigma(B) \subset i \mathbb{R}$, and since for every $\lambda \in i \mathbb{R}$ the function $g$, defined by

$$
g(t):=\frac{e^{\lambda t}}{t^{\alpha}} \chi_{[1, \infty)}(t) \quad(t \in \mathbb{R})
$$

with arbitrary $\alpha \in\left(\frac{1}{p}, 1+\frac{1}{p}\right.$ ], belongs to $L^{p}(\mathbb{R})$ but not to the range of $B-\lambda$, one even has $\sigma(B)=i \mathbb{R}$ for $p \in[1, \infty)$. Additionally, since $B_{q}^{*}=-B_{q^{*}}$ for every $q \in[1, \infty)$ with dual exponent $q^{*}$ and since $\sigma_{p}\left(B_{q}\right)=\emptyset$ for $q \in[1, \infty)$ and $\sigma_{p}\left(B_{q}\right)=i \mathbb{R}$ for $q=\infty$, one obtains the following fine structure of the spectrum of $B$ :

$$
\begin{gathered}
\sigma_{p}(B)=\emptyset, \quad \sigma_{c}(B)=\emptyset, \quad \sigma_{r}(B)=i \mathbb{R} \quad(p=1) \\
\sigma_{p}(B)=\emptyset, \quad \sigma_{c}(B)=i \mathbb{R}, \quad \sigma_{r}(B)=\emptyset \quad(p \in(1, \infty)) .
\end{gathered}
$$

Example 5.8. Suppose $A, \lambda, P$ with $A(t)=R(t)^{-1} A_{0}(t) R(t), P(t)=R(t)^{-1} P_{0} R(t)$, and $R(t)=e^{C t}$ are given as follows in $X:=\ell^{p}\left(I_{d}\right) \times L^{p}(\mathbb{R})$ (where $p \in(1, \infty)$ and $d \in \mathbb{N}$ ):

$$
A_{0}(t):=\left(\begin{array}{cc}
\lambda(t)+\alpha(t) N & 0 \\
0 & B
\end{array}\right) \quad \text { and } \quad P_{0}:=\left(\begin{array}{ll}
1 & 0 \\
0 & 0
\end{array}\right)
$$

where $\lambda(t) \in\{\operatorname{Re} z \leq 0\}, \alpha(t), N$ are such that Condition 2.16 is satisfied and where $B$ is the differentiation operator on $L^{p}(\mathbb{R})$ defined above, so that, in particular,

$$
D\left(A_{0}(t)\right)=D:=\ell^{p}\left(I_{d}\right) \times W^{1, p}(\mathbb{R}) .
$$


Additionally, suppose $t \mapsto \lambda(t), \alpha(t)$ are continuously differentiable and $C$ is the bounded linear map in $\ell^{p}\left(I_{d}\right) \times L^{p}(\mathbb{R})$ given by

$$
C:=\left(\begin{array}{cc}
0 & 0 \\
C_{0} & 0
\end{array}\right) \quad \text { with } \quad C_{0}\left(x_{1}, \ldots, x_{d}\right):=x_{d} f_{0}
$$

for an arbitrary fixed $0 \neq f_{0} \in L^{p}(\mathbb{R})$. Since (by $p \neq 1$ ) the spectrum of $\left.A_{0}(t)\right|_{\left(1-P_{0}\right) D}=$ $B$ is purely continuous for every $t \in I, P_{0}$ is weakly associated with $A_{0}(t)$ and $P_{0}$, and hence the same is true for $A(t)$ and $P(t)$ instead of $A_{0}(t), P_{0}$. Since, moreover, $B$ generates a contraction group (not only a semigroup) in $L^{p}(\mathbb{R})$, the resolvent estimates of Theorem 5.4 are satisfied with $\vartheta(t):=\pi$.

It follows in the same way as after Example 4.7 that $A, \lambda, P$ of the above example cannot be reduced to a finite-dimensional subspace and that our adiabatic theorem without spectral gap condition does not apply if $p=1$ in the example above. Also, it should be noticed that the domains $D(A(t))=e^{-C t} D$ of the above $A(t)$ really are time-dependent - more precisely, one has $D\left(A\left(t_{1}\right)\right) \neq D\left(A\left(t_{2}\right)\right)$ for $t_{1} \neq t_{2}$ - if only $f_{0}$ is chosen to lie not in $W^{1, p}(\mathbb{R})$. Indeed, if under this condition on $f_{0}$ one has the twofold representation

$$
\left(x, f-t_{1} x_{d} f_{0}\right)=e^{-C t_{1}}(x, f)=e^{-C t_{2}}(y, g)=\left(y, g-t_{2} y_{d} f_{0}\right)
$$

for some $(x, f),(y, g) \in \ell^{p}\left(I_{d}\right) \times W^{1, p}(\mathbb{R})=D$ with $x_{d} \neq 0$, then $t_{1}$ must be equal to $t_{2}$.

\section{Adiabatic theorems for operators defined by symmetric sesquilinear forms}

After having established general adiabatic theorems for time-dependent domains in Section 5, we now apply these theorems to obtain - as simple corollaries - adiabatic theorems for operators $A(t)=i A_{a(t)}$ defined by densely defined closed symmetric sesquilinear forms $a(t)$ with time-independent form domain - such as, for instance, Schrödinger operators $A(t)$ with time-dependent potentials $V(t)$ belonging to the Rollnik class. In particular, the theorem of Section 6.3 contains the adiabatic theorem of Bornemann from [13] as a special case.

\subsection{Some notation and preliminaries}

We start by recording the basic conditions (depending on a regularity parameter $n \in$ $\mathbb{N} \cup\{\infty\})$ that shall be imposed on the sesquilinear forms $a(t)$ in the adiabatic theorems of this section.

Condition 6.1. $a(t)$ for every $t \in I$ is a symmetric sesquilinear form on the Hilbert space $H^{+}$(with norm $\|.\|^{+}$and scalar product $\langle., . .\rangle^{+}$) which is densely and continuously embedded into $H$ (with norm $\|$.$\| and scalar product \langle., .$.$\rangle ). There is a number m \in$ $(0, \infty)$ such that

$$
\langle., . .\rangle_{t}^{+}:=a(t)(., . .)+m\langle., . .\rangle
$$


is a scalar product on $H^{+}$and such that the induced norm $\|\cdot\|_{t}^{+}$is equivalent to $\|\cdot\|^{+}$ for every $t \in I$. And finally, $t \mapsto a(t)(x, y)$ is $n$ times continuously differentiable for all $x, y \in H^{+}$.

In Condition 6.1, the requirement that $\langle., . .\rangle_{t}^{+}$be a scalar product on $H^{+}$whose norm $\|\cdot\|_{t}^{+}$is equivalent to $\|\cdot\|^{+}$for every $t \in I$ could be reformulated in an equivalent way by saying that there is $m \in(0, \infty)$ such that $a(t)(., .)+.m\langle., .$.$\rangle is \|\cdot\|^{+}$-bounded and $\|\cdot\|^{+}$-coercive. It is well-known that under Condition 6.1 there is, for every $t \in I$, a unique self-adjoint operator $A_{a(t)}: D\left(A_{a(t)}\right) \subset H \rightarrow H$ such that

$$
D\left(A_{a(t)}\right) \subset H^{+} \quad \text { and } \quad\left\langle x, A_{a(t)} y\right\rangle=a(t)(x, y)
$$

for every $x \in H^{+}$and $y \in D\left(A_{a(t)}\right)$ (Theorem VI.2.1 and Theorem VI.2.6 of [31] or Theorem 10.1.2 of [10]). As usual, we denote - in the situation of Condition 6.1 - by $H^{-}$ the space of $\|\cdot\|^{+}$-continuous conjugate linear functionals $H^{+} \rightarrow \mathbb{C}$, which obviously is a Hilbert space w.r.t. the norms

$$
f \mapsto\|f\|^{-}:=\sup _{\|x\|^{+}=1}|f(x)| \text { and } f \mapsto\|f\|_{t}^{-}:=\sup _{\|x\|_{t}^{+}=1}|f(x)| \quad(t \in I) .
$$

And by $j: H \rightarrow H^{-}$we denote the injective continuous linear map with $j(x):=\langle., x\rangle \in$ $H^{-}$for $x \in H$.

We continue by citing the fundamental theorem of Kisyński (Theorem 8.1 of [34]) giving sufficient conditions for the well-posedness of the initial value problems corresponding to $A$ on $D(A(t))$, where $A(t)=i A_{a(t)}$ with symmetric sesquilinear forms $a(t)$ with constant domain. Similar theorems on well-posedness can be proved for the case of operators $A(t)=-A_{a(t)}$ defined by sectorial sesquilinear forms $a(t)$ with time-independent form domain. See, for instance, Fujie and Tanabe's article [21] (Theorem 3.1) or Kato and Tanabe's article [28] (Theorem 7.3).

Theorem 6.2 (Kisyński). Suppose a $(t)$ for every $t \in I$ is a sesquilinear form such that Condition 6.1 is satisfied with $n=2$ and set $A(t):=i A_{a(t)}$ for $t \in I$. Then there is a unique evolution system $U$ for $A$ on $D(A(t))$ and $U(t, s)$ is unitary in $H$ for every $(s, t) \in \Delta$.

In particular, this theorem guarantees that the basic Condition 5.1 of the general adiabatic theorems for time-dependent domains is satisfied if Condition 6.1 is with $n=$ 2. When it comes to verifying the other conditions of the general adiabatic theorems discussed in Section 5, the following lemma will be important.

Lemma 6.3. Suppose that Condition 6.1 is satisfied for a certain $n \in \mathbb{N}$ and, for every $t \in I$, denote by $\tilde{A}_{0}(t)$ the bounded linear map $H^{+} \rightarrow H^{-}$extending $A_{0}(t):=A_{a(t)}$, that is, $\tilde{A}_{0}(t) x:=a(t)(., x)$ for $x \in H^{+}$. Then the following holds true:

(i) $t \mapsto \tilde{A}_{0}(t)$ is $n$ times WOT-continuously differentiable. 
(ii) If for a certain $z \in \mathbb{C}$ the operator $A_{0}(t)-z: D\left(A_{0}(t)\right) \subset H \rightarrow H$ is bijective for all $t \in J_{0}$ (a non-trivial subinterval of $I$ ), then so is $\tilde{A}_{0}(t)-z j: H^{+} \rightarrow H^{-}$and

$$
\left(A_{0}(t)-z\right)^{-1} x=\left(\tilde{A}_{0}(t)-z j\right)^{-1} j(x)
$$

for all $t \in J_{0}$ and $x \in H$. In particular, $J_{0} \ni t \mapsto\left(A_{0}(t)-z\right)^{-1}$ is $n$ times WOT-continuously differentiable.

Proof. (i) We have only to show that $t \mapsto F\left(\tilde{A}_{0}(t) x\right)$ is $n$ times continuously differentiable for every $x \in H^{+}$and every $F \in\left(H^{-}\right)^{*}$. Since the canonical conjugate linear map

$$
H^{+} \ni y \mapsto i(y) \in\left(H^{-}\right)^{*} \text { with } i(y)(f):=f(y) \text { for } f \in H^{-}
$$

is surjective by the reflexivity of $H^{+}$, the claim is obvious from the continuous differentiability requirement in Condition 6.1.

(ii) We fix $t \in I$ and show that

$$
\rho\left(A_{0}(t)\right) \subset \rho\left(A_{0}^{-}(t)\right)
$$

where $A_{0}^{-}(t): j\left(H^{+}\right) \subset H^{-} \rightarrow H^{-}$is defined by $A_{0}^{-}(t) j(x)=\tilde{A}_{0}(t) x$ for $x \in H^{+}$. Since $A_{0}^{-}(t)$ is self-adjoint in $\left(H^{-},\|\cdot\|_{t}^{-}\right)$, it follows that $\mathbb{C} \backslash \mathbb{R} \subset \rho\left(A_{0}^{-}(t)\right)$ and that

$$
\left(A_{0}^{-}(t)-z\right)^{-1} j(x)=j\left(\left(A_{0}(t)-z\right)^{-1} x\right)
$$

for $z \in \mathbb{C} \backslash \mathbb{R}$ and $x \in H$. It therefore remains to prove that $\rho\left(A_{0}(t)\right) \cap \mathbb{R} \subset \rho\left(A_{0}^{-}(t)\right)$. So let $z \in \rho\left(A_{0}(t)\right) \cap \mathbb{R}$. Then there is $\delta>0$ such that $(z-2 \delta, z+2 \delta) \subset \rho\left(A_{0}(t)\right)$, from which it follows by Stone's formula (applied to both $A_{0}(t)$ and $A_{0}^{-}(t)$ ) and by (6.2) that

$$
0=j\left(P_{(z-\delta, z+\delta)} x+\frac{1}{2} P_{\{z-\delta, z+\delta\}} x\right)=\left(P_{(z-\delta, z+\delta)}^{-}+\frac{1}{2} P_{\{z-\delta, z+\delta\}}^{-}\right) j(x)
$$

for all $x \in H$, where $P$ and $P^{-}$denote the spectral measure of $A_{0}(t)$ and $A_{0}^{-}(t)$, respectively. It follows (by the density of $j(H)$ in $H^{-}$) that $P_{(z-\delta, z+\delta)}^{-}=0$ and hence $z \in \rho\left(A_{0}^{-}(t)\right)$. So (6.1) is established and the desired conclusion ensues.

\subsection{Adiabatic theorems with spectral gap condition}

We will need the following condition depending on a parameter $m \in\{0\} \cup \mathbb{N} \cup\{\infty\}$ for the adiabatic theorem with spectral gap condition below.

Condition 6.4. $A(t)=i A_{a(t)}$ for $t \in I$, where the sesquilinear forms a $(t)$ satisfy Condition 6.1 with $n=2 . \sigma(t)$ for every $t \in I$ is a compact subset of $\sigma(A(t)), \sigma($.$) falls$ into $\sigma(A().) \backslash \sigma($.$) at exactly m$ points that accumulate at only finitely many points, and $I \backslash N \ni t \mapsto \sigma(t)$ is continuous, where $N$ denotes the set of those $m$ points at which $\sigma($. falls into $\sigma(A().) \backslash \sigma($.$) . P(t)$ for every $t \in I \backslash N$ is the projection associated with $A(t)$ and $\sigma(t)$ and $I \backslash N \ni t \mapsto P(t)$ extends to a twice SOT-continuously differentiable map (again denoted by $P$ ) on the whole of $I$. 
In view of Lemma 6.3 it is now very easy to derive the following adiabatic theorem with uniform $(m=0)$ or non-uniform $(m \in \mathbb{N} \cup\{\infty\})$ spectral gap condition from the corresponding general adiabatic theorem with spectral gap condition (Theorem 5.3).

Theorem 6.5. Suppose $A(t), \sigma(t), P(t)$ for $t \in I$ are as in Condition 6.4 with $m=0$ or $m \in \mathbb{N} \cup\{\infty\}$, respectively. Then

$$
\sup _{t \in I}\left\|U_{\varepsilon}(t)-V_{\varepsilon}(t)\right\|=O(\varepsilon) \text { resp. o(1) } \quad(\varepsilon \searrow 0),
$$

whenever the evolution system $V_{\varepsilon}$ for $\frac{1}{\varepsilon} A+\left[P^{\prime}, P\right]$ exists on $D(A(t))$ for every $\varepsilon \in(0, \infty)$.

Proof. Choose, for every $t_{0} \in I \backslash N$, non-trivial closed intervals $J_{t_{0}}$ and cycles $\gamma_{t_{0}}$ as in Condition 5.2 (which is possible by the relative openness of $I \backslash N$ in $I$ ). In virtue of Lemma 6.3 it is then clear that Condition 5.2 is fulfilled, and the assertion follows from Theorem 5.3 .

If the existence of the evolution $V_{\varepsilon}$ for $\frac{1}{\varepsilon} A+\left[P^{\prime}, P\right]$ cannot be ensured, one still has the remark after Theorem 5.3. In the case of uniform spectral gap, the existence of $V_{\varepsilon}$ is guaranteed if, for instance, Condition 6.1 is fulfilled with $n=3$, since then $I \ni$ $t \mapsto P(t)$ is thrice WOT-continuously differentiable (by Lemma 6.3 (ii)) so that the symmetric sesquilinear forms $\frac{1}{\varepsilon} a(t)+b(t)=\frac{1}{\varepsilon} a(t)-i\left\langle.,\left[P^{\prime}(t), P(t)\right] ..\right\rangle$ corresponding to $\frac{1}{\varepsilon} A(t)+\left[P^{\prime}(t), P(t)\right]$ satisfy Condition 6.1 with $n=2$ and Theorem 6.2 can be applied.

We finally note conditions under which the general adiabatic theorem of higher order (Theorem 5.7) can be applied to the case of operators $A(t)$ defined by symmetric sesquilinear forms.

Condition 6.6. Suppose that $A(t)=i A_{a(t)}$ for $t \in I$ where the sesquilinear forms $a(t)$ satisfy Condition 6.1 with a certain $n \in \mathbb{N} \backslash\{1\}$ or with $n=\infty$, respectively. In the latter case suppose further that there is an open neighbourhood $U_{I}$ of $I$ in $\mathbb{C}$ and for each $w \in U_{I}$ there is a $\|\cdot\|^{+}$-bounded sesquilinear form $\tilde{a}(w)$ on $H^{+}$such that $\tilde{a}(t)=a(t)$ for $t \in I$ and that $U_{I} \ni w \mapsto \tilde{a}(w)(x, y)$ is holomorphic for every $x, y \in H^{+}$. Suppose moreover that $\sigma(t)$ for every $t \in I$ is an isolated compact subset of $\sigma(A(t))$, that $\sigma($.$) at$ no point falls into $\sigma(A().) \backslash \sigma($.$) , and that t \mapsto \sigma(t)$ is continuous. And finally, suppose $P(t)$ for every $t \in I$ is the projection associated with $A(t)$ and $\sigma(t)$ and $t \mapsto P(t)$ is $n+1$ times times SOT-continuously differentiable.

It is not difficult (albeit a bit technical) to show that under Condition 6.6 the hypotheses of Theorem 5.7 are really satisfied. (In the case $n=\infty$ define $\tilde{A}_{0}(w)$ by $\tilde{A}_{0}(w) x:=\tilde{a}(w)(., x)$ for $x \in H^{+}$. Then $\tilde{A}_{0}(w)$ is a bounded linear map $H^{+} \rightarrow H^{-}$and $U_{I} \ni w \mapsto \tilde{A}_{0}(w) \in L\left(H^{+}, H^{-}\right)$is WOT-holomorphic and hence NOT-holomorphic. A simple perturbation argument and Cauchy's inequality (in conjunction with the formula in Lemma 6.3 (ii)) then yield estimates of the desired kind.)

\subsection{An adiabatic theorem without spectral gap condition}

In the adiabtic theorem without spectral gap condition below, the following condition will be used. 
Condition 6.7. $A(t)=i A_{a(t)}$ for $t \in I$ where the sesquilinear forms a $(t)$ satisfy Condition 6.1] with $n=2$. $\lambda(t)$ for every $t \in I$ is an eigenvalue of $A(t)$ such that $t \mapsto \lambda(t)$ is continuous. And $P(t)$ for every $t \in I$ is an orthogonal projection in $H$ such that $P(t)$ is weakly associated with $A(t)$ and $\lambda(t)$ for almost every $t \in I, \operatorname{rk} P(0)<\infty$ and $t \mapsto P(t)$ is SOT-continuously differentiable.

While in the case with spectral gap Lemma 6.3 was sufficient, we need another well-expected - lemma in the case without spectral gap.

Lemma 6.8. Suppose that Condition 6.7 is satisfied and that, in addition, $t \mapsto \lambda(t)$ is continuously differentiable. Then $t \mapsto(\lambda(t)+\delta-A(t))^{-1}$ is SOT-continuously differentiable for every $\delta \in(0, \infty)$ and there is an $M_{0}^{\prime} \in(0, \infty)$ such that

$$
\left\|\frac{d}{d t}(\lambda(t)+\delta-A(t))^{-1}\right\| \leq \frac{M_{0}^{\prime}}{\delta^{2}}
$$

for all $t \in I$ and $\delta \in(0,1]$.

Proof. Set $A_{0}(t):=A_{a(t)}=-i A(t)$ and $\lambda_{0}(t):=-i \lambda(t)$ and let $\tilde{A}_{0}(t): H^{+} \rightarrow H^{-}$ be the bounded extension of $A_{0}(t)$. Since by Lemma 6.3 $t \mapsto \tilde{A}_{0}(t)$ is twice WOTand, in particular, once SOT-continuously differentiable and $t \mapsto \lambda_{0}(t)$ is continuously differentiable, it follows that

$$
t \mapsto\left(A_{0}(t)-\left(\lambda_{0}(t)-i \delta\right)\right)^{-1}=\left(\tilde{A}_{0}(t)-\left(\lambda_{0}(t)-i \delta\right) j\right)^{-1} j
$$

is SOT-continuously differentiable for every $\delta \in(0, \infty)$ and that

$$
\begin{aligned}
& \frac{d}{d t}\left(A_{0}(t)-\left(\lambda_{0}(t)-i \delta\right)\right)^{-1} \\
& \quad=\left(\tilde{A}_{0}(t)-\left(\lambda_{0}(t)-i \delta\right) j\right)^{-1}\left(\lambda_{0}^{\prime}(t) j-\tilde{A}_{0}^{\prime}(t)\right)\left(\tilde{A}_{0}(t)-\left(\lambda_{0}(t)-i \delta\right) j\right)^{-1} j
\end{aligned}
$$

for $t \in I$ and $\delta \in(0, \infty)$. We therefore show that there is a constant $c_{0}^{\prime} \in(0, \infty)$ such that

$$
\left\|\left(\tilde{A}_{0}(t)-\left(\lambda_{0}(t)-i \delta\right) j\right) x\right\|_{t}^{-} \geq \frac{\delta}{c_{0}^{\prime}}\|x\|_{t}^{+}
$$

for all $x \in H^{+}, t \in I$ and $\delta \in(0,1]$. In order to do so we observe the following simple fact: if instead of $j$ the natural isometric isomorphism

$$
j_{t}^{+}:\left(H^{+},\|\cdot\|_{t}^{+}\right) \rightarrow\left(H^{-},\|\cdot\|_{t}^{-}\right) \text {with } j_{t}^{+}(x):=\langle., x\rangle_{t}^{+} \text {for } x \in H^{+}
$$

occurred in (6.4), this assertion would be trivial. We are therefore led to express $j$ in terms of $j_{t}^{+}$: by the definition of the scalar product $\langle., . .\rangle_{t}^{+}$in Condition 6.1, we have

$$
j=\frac{1}{m}\left(\tilde{A}_{0}(t)-j_{t}^{+}\right)
$$


for all $t \in I$, so that

$$
\tilde{A}_{0}(t)-\left(\lambda_{0}(t)-i \delta\right) j=\frac{m+\lambda_{0}(t)-i \delta}{m}\left(\tilde{A}_{0}(t)-\frac{\lambda_{0}(t)-i \delta}{m+\lambda_{0}(t)-i \delta} j_{t}^{+}\right) .
$$

Since for all $x \in H^{+}$with $\|x\|_{t}^{+}=1$

$$
\begin{aligned}
\left\|\left(\tilde{A}_{0}(t)-\frac{\lambda_{0}(t)-i \delta}{m+\lambda_{0}(t)-i \delta} j_{t}^{+}\right) x\right\|_{t}^{-} & \geq\left|a(t)(x, x)-\frac{\lambda_{0}(t)-i \delta}{m+\lambda_{0}(t)-i \delta}\left(j_{t}^{+}(x)\right)(x)\right| \\
& \geq\left|\operatorname{Im}\left(\frac{\lambda_{0}(t)-i \delta}{m+\lambda_{0}(t)-i \delta}\right)\right|=\frac{m \delta}{\left|m+\lambda_{0}(t)-i \delta\right|^{2}},
\end{aligned}
$$

it follows that

$$
\left\|\left(\tilde{A}_{0}(t)-\left(\lambda_{0}(t)-i \delta\right) j\right) x\right\|_{t}^{-} \geq\left|\frac{m+\lambda_{0}(t)-i \delta}{m}\right| \frac{m \delta}{\left|m+\lambda_{0}(t)-i \delta\right|^{2}}\|x\|_{t}^{+} \geq \frac{\delta}{c_{0}^{\prime}}\|x\|_{t}^{+}
$$

for all $x \in H^{+}$and all $t \in I, \delta \in(0,1]$, where $c_{0}^{\prime}:=m+\|\lambda\|_{\infty}+1$. So (6.4) is proven and it follows that

$$
\left\|\left(\tilde{A}_{0}(t)-\left(\lambda_{0}(t)-i \delta\right) j\right)^{-1}\right\|_{H^{-} \rightarrow H^{+}} \leq \frac{c_{0}^{\prime}}{\delta}
$$

for all $t \in I$ and $\delta \in(0,1]$, because the equivalence of the norms $\|\cdot\|_{t}^{+}$with $\|\cdot\|$ required in Condition 6.1 is uniform w.r.t. $t$ by Lemma 7.3 of [34. In view of (6.3) and (6.5) the asserted estimate is now clear.

With this lemma at hand, it is now simple to derive the following adiabatic theorem without spectral gap condition which generalizes an adiabatic theorem of Bornemann (Theorem IV.1 of [13]).

Theorem 6.9. Suppose $A(t), \lambda(t), P(t)$ for $t \in I$ are such that Condition 6.7 is satisfied. Then

$$
\sup _{t \in I}\left\|\left(U_{\varepsilon}(t)-V_{0 \varepsilon}(t)\right) P(0)\right\| \longrightarrow 0 \quad \text { and } \quad \sup _{t \in I}\left\|P(t)\left(U_{\varepsilon}(t)-V_{0 \varepsilon}(t)\right)\right\| \longrightarrow 0
$$

as $\varepsilon \searrow 0$, where $V_{0 \varepsilon}$ denotes the evolution system for $\frac{1}{\varepsilon} A P+\left[P^{\prime}, P\right]=\frac{1}{\varepsilon} \lambda P+\left[P^{\prime}, P\right]$ for every $\varepsilon \in(0, \infty)$. If, in addition, $t \mapsto P(t)$ is thrice WOT-continuously differentiable, then the evolution system $V_{\varepsilon}$ for $\frac{1}{\varepsilon} A+\left[P^{\prime}, P\right]$ exists on $D(A(t))$ for every $\varepsilon \in(0, \infty)$ and

$$
\sup _{t \in I}\left\|U_{\varepsilon}(t)-V_{\varepsilon}(t)\right\| \longrightarrow 0 \quad(\varepsilon \searrow 0) .
$$

Proof. We have to verify the hypotheses of the general adiabatic theorem without spectral gap condition for time-dependent domains (Theorem 5.4) with $m_{0}=1$. In view of Lemma 6.8 it remains to establish two small things, namely the continuous differentiability of $t \mapsto \lambda(t)$ (from Theorem 5.4 and from Lemma 6.8) and the inclusion 
$P(t) H \subset \operatorname{ker}(A(t)-\lambda(t))$ for every $t \in I$ (from Theorem 5.4). We know by assumption that $P(t) H=\operatorname{ker}(A(t)-\lambda(t))=\operatorname{ker}\left(A_{0}(t)-\lambda_{0}(t)\right)$ for almost every $t \in I$ so that $P(t) H \subset D\left(A_{0}(t)\right) \subset H^{+}$and

$$
0=j\left(\left(A_{0}(t)-\lambda_{0}(t)\right) P(t) x\right)=\left(A_{0}^{-}(t)-\lambda_{0}(t)\right) j(P(t) x)
$$

for all $x \in H$ and almost every $t \in I$ (where $A_{0}(t), \lambda_{0}(t)$ are defined as in the proof of Lemma 6.8 and where $A_{0}^{-}(t)$ is the self-adjoint operator in $\left(H^{-},\|\cdot\|_{t}^{-}\right)$from the proof of Lemma 6.3). Applying the closedness argument after Theorem 3.2 to the closed operator $i A_{0}^{-}(t): j\left(H^{+}\right) \subset H^{-} \rightarrow H^{-}$(with time-independent domain!), we see that $j(P(t) H) \subset j\left(H^{+}\right)$and

$$
0=\left(A_{0}^{-}(t)-\lambda_{0}(t)\right) j(P(t) x)=a(t)(., P(t) x)-\lambda_{0}(t)\langle., P(t) x\rangle
$$

for all $x \in H$ and every (not only almost every) $t \in I$. In particular, for every $t \in I$,

$$
0=a(t)(y, P(t) x)-\lambda_{0}(t)\langle y, P(t) x\rangle=\left\langle\left(A_{0}(t)-\lambda_{0}(t)\right) y, P(t) x\right\rangle
$$

for $y \in D\left(A_{0}(t)\right)$ and $x \in H$, so that

$$
P(t) H \subset \operatorname{ker}\left(A_{0}(t)-\lambda_{0}(t)\right)^{*}=\operatorname{ker}(A(t)-\lambda(t))
$$

for every $t \in I$, as desired. Since for every $t_{0} \in I$ there is a neighbourhood $J_{t_{0}} \subset I$ and an $x_{0} \in H$ such that $P(t) x_{0} \neq 0$ for $t \in J_{t_{0}}$, it follows from (6.6) that

$$
\frac{1}{\lambda(t)-1}=\frac{\left\langle P(t) x_{0},(A(t)-1)^{-1} P(t) x_{0}\right\rangle}{\left\langle P(t) x_{0}, P(t) x_{0}\right\rangle}
$$

for every $t \in J_{t_{0}}$, from which in turn it follows (by Lemma 6.3) that $t \mapsto \lambda(t)$ is continuously differentiable, as desired.

According to what has been said at the beginning of the proof, it is now clear that Lemma 6.8 can be applied and that the hypotheses of the first part of Theorem 5.4 are satisfied. Since the evolution system $U_{\varepsilon}$ is unitary (by Theorem 6.2) and $V_{0 \varepsilon}$ is unitary as well, we see by obviously modifying the proof of Theorem 5.4 that

$$
\sup _{(s, t) \in I^{2}}\left\|\left(U_{\varepsilon}(t, s)-V_{0 \varepsilon}(t, s)\right) P(s)\right\| \longrightarrow 0 \quad(\varepsilon \searrow 0)
$$

where $U_{\varepsilon}(t, s):=U_{\varepsilon}(s, t)^{-1}=U_{\varepsilon}(s, t)^{*}$ and $V_{0 \varepsilon}(t, s):=V_{0 \varepsilon}(s, t)^{-1}=V_{0 \varepsilon}(s, t)^{*}$ for $(s, t) \in I^{2}$ with $s>t$. Since

$$
\left\|P(t)\left(U_{\varepsilon}(t)-V_{0 \varepsilon}(t)\right)\right\|=\left\|\left(U_{\varepsilon}(0, t)-V_{0 \varepsilon}(0, t)\right) P(t)\right\|
$$

for $t \in I$ (take adjoints), the first two of the asserted convergences follow from (6.7).

Suppose finally that $t \mapsto P(t)$ is thrice WOT-continuously differentiable. Then the symmetric sesquilinear forms $\frac{1}{\varepsilon} a(t)+b(t)=\frac{1}{\varepsilon} a(t)-i\left\langle.,\left[P^{\prime}(t), P(t)\right] ..\right\rangle$ corresponding to the operators $\frac{1}{\varepsilon} A(t)+\left[P^{\prime}(t), P(t)\right]$ satisfy Condition 6.1 with $n=2$ and therefore the evolution system $V_{\varepsilon}$ for $\frac{1}{\varepsilon} A+[P, P]$ exists on $D(A(t))$ for every $\varepsilon \in(0, \infty)$ by Theorem 6.2 . Also, $t \mapsto P(t)$ is obviously norm continuously differentiable and so the hypotheses of the second part of Theorem 5.4 are satisfied, which gives the last convergence. 
What are the differences between the above theorem and Bornemann's adiabatic theorem of [13]? While in Theorem IV.1 of [13] $\lambda(t)$ is required to belong to the discrete spectrum of $A(t)$ (and hence to be an isolated eigenvalue) for every $t \in I$, in the above theorem it is only required that $\lambda(t)$ has finite multiplicity for almost every $t \in I$ : the eigenvalues $\lambda(t)$ are allowed to have infinite multiplicity on a set of measure zero and, moreover, they are allowed to be non-isolated in $\sigma(A(t))$ for every $t \in I$. Also, the regularity conditions on $A$ and $P$ of the above theorem are slightly weaker than those of Theorem IV.1: for instance, $t \mapsto \tilde{A}_{0}(t)$ is required to be twice NOT-continuously differentiable in [13] whereas above it is only required that $t \mapsto a(t)(x, y)$ be twice continuously differentiable for $x, y \in H^{+}$(or equivalently (Lemma 6.3), that $t \mapsto \tilde{A}_{0}(t)$ be twice WOT-continuously differentiable). And finally, the statement of the theorem above is a bit more general than the conclusion of Theorem IV.1 in [13] which says that, for all $x \in H^{+}$(and hence for all $x \in H$ ) and uniformly in $t \in I$,

$$
\begin{aligned}
\left\langle U_{\varepsilon}(t) x, P(t) U_{\varepsilon}(t) x\right\rangle & =\left\langle U_{\varepsilon}(t) x, P(t) U_{\varepsilon}(t) x-U_{\varepsilon}(t) P(0) x\right\rangle+\langle x, P(0) x\rangle \\
& \longrightarrow\langle x, P(0) x\rangle \quad(\varepsilon \searrow 0) .
\end{aligned}
$$

Acknowledgement: I would like to thank Professor Marcel Griesemer very much for useful discussions and for having directed my interest towards adiabatic theory in the first place.

\section{References}

[1] W. Abou Salem, J. Fröhlich: Adiabatic theorems and reversible isothermal processes. Lett. Math. Phys. 72 (2005), 153-163.

[2] W. Abou Salem: On the quasi-static evolution of nonequilibrium steady states. Ann. Henri Poincaré 8 (2007), 569-596.

[3] W. Abou Salem, J. Fröhlich: Adiabatic theorems for quantum resonances. Comm. Math. Phys. 237 (2007), 651-675.

[4] P. Acquistapace, B. Terreni: A unified approach to abstract linear nonautonomous parabolic equations. Rend. Sem. Mat. Univ. Padova 78 (1987), 47-107.

[5] H. Amann: Linear and quasilinear parabolic problems I. Abstract linear theory. Birkhäuser, 1995. 
[6] W. Arendt, C. Batty, M. Hieber, F. Neubrander: Vector-valued Laplace transforms and Cauchy problems. Birkhäuser, 2001.

[7] J.E. Avron, A. Elgart: Adiabatic theorem without a gap condition. Commun. Math. Phys. 203 (1999), 445-463.

[8] J.E. Avron, M. Fraas, G.M. Graf, P. Grech: Adiabatic theorems for generators of contracting evolutions. ArXiv preprint, 2011.

[9] J.E. Avron, R. Seiler, L.G. Yaffe: Adiabatic theorems and applications to the quantum Hall effect. Commun. Math. Phys. 110 (1987), 33-49. (In conjunction with the corresponding erratum of 1993.)

[10] M.S. Birman, M.Z. Solomjak: Spectral theory of self-adjoint operators in Hilbert space. Kluwer, 1987.

[11] J. Blank, P. Exner, M. Havlíček: Hilbert space operators in quantum physics. American Institute of Physics Press, 1994.

[12] M. Born, V. Fock: Beweis des Adiabatensatzes. Z. Phys. 51 (1928), 165-180.

[13] F. Bornemann: Homogenization in time of singularly perturbed mechanical systems. Lecture Notes in Mathematics 1687, Springer 1998.

[14] C. Brouder, G. Panati, G. Stoltz: Gell-Mann and Low formula for degenerate unperturbed states. Ann. Henri Poincaré 10 (2010), 1285-1309.

[15] J. B. Conway: A Course in Functional Analysis. 2nd edition. Springer, 1990.

[16] J. L. Daleckii, S. G. Krein: On differential equations in Hilbert space. Ukrain. Mat. Z. 2 (1950), 71-91.

[17] N. Dunford: A survey of the theory of spectral operators. Bull. Amer. Math. Soc. 64 (1958), 217-274.

[18] N. Dunford, J. T. Schwartz: Linear operators I-III. Wiley, 1958, 1963, 1971.

[19] A. Elgart, G. A. Hagedorn: An adiabatic theorem for resonances. Comm. Pure Appl. Math. 64 (2011), 1029-1058.

[20] K.-J. Engel, R. Nagel: One-parameter semigroups for linear evolution equations. Springer, 2000.

[21] Y. Fujie, H. Tanabe: On some parabolic equations of evolution in Hilbert space. Osaka J. Math. 10 (1973), 115-130.

[22] F. Gesztesy, V. Tkachenko: A criterion for Hill operators to be spectral operators of scalar type. J. Anal. Math. 107 (2009), 287-353. 
[23] I. Gohberg, S. Goldberg, M. A. Kaashoek: Classes of linear operators I-II. Birkhäuser, 1990, 1993.

[24] A. Joye, C.-E. Pfister: Superadiabatic evolution and adiabatic transition probability between two non-degenerate levels isolated in the spectrum. J. Math. Phys. 34 (1993), 454-479.

[25] A. Joye: General adiabatic evolution with a gap condition. Commun. Math. Phys. 275 (2007), 139-162.

[26] T. Kato: On the Adiabatic Theorem of Quantum Mechanics. J. Phys. Soc. Japan 5 (1950), 435-439.

[27] T. Kato: On linear differential equations in Banach spaces. Comm. Pure Appl. Math. 9 (1956), 479-486.

[28] T. Kato, H. Tanabe: On the abstract evolution equation. Osaka Math. J. 14 (1962), 107-133.

[29] T. Kato: Linear evolution equations of "hyperbolic" type. J. Fac. Sci. Univ. Tokyo 17 (1970), 241-258.

[30] T. Kato: Linear evolution equations of "hyperbolic" type II. J. Math. Soc. Japan 25 (1973), 648-666.

[31] T. Kato: Perturbation theory for linear operators. 2nd edition. Springer, 1980.

[32] T. Kato: Abstract differential equations and nonlinear mixed problems. Lezioni Fermiane, Accademia Nazionale dei Lincei, Scuola Normale Superiore, Pisa (1985), $1-89$.

[33] J. v. Keler, S. Teufel: Non-adiabatic transitins in a massless scalar field. ArXiv preprint, 2012.

[34] J. Kisyński: Sur les opérateurs de Green des problèmes de Cauchy abstraits. Stud. Math. 23 (1963), 285-328.

[35] S. G. Krein: Linear differential equations in Banach space. Transl. Math. Monographs, American Mathematical Society, 1971.

[36] D. C. Lay: Spectral analysis using ascent, descent, nullity and defect. Math. Ann. 184 (1970), 197-214.

[37] G. Lumer, R. S. Phillips: Dissipative operators in a Banach space. Pacific J. Math. 11 (1961) 679-698.

[38] G. Nenciu: On the adiabatic theorem of quantum mechanics. J. Phys. A: Math. Gen. 13 (1980), 15-18. 
[39] G. Nenciu, G. Rasche: On the adiabatic theorem for non-self-adjoint Hamiltonians. J. Phys. A: Math. Gen. 25 (1992), 5741-5751.

[40] G. Nenciu: Linear adiabatic theory. Exponential estimates. Commun. Math. Phys. 152 (1993), 479-496.

[41] G. Nickel, R. Schnaubelt: An extension of Kato's stability condition for nonautonomous Cauchy problems. Taiw. J. Math. 2 (1998), 483-496.

[42] G. Nickel: Evolution semigroups and product formulas for nonautonomous Cauchy problems. Math Nachr. 212 (2000), 101-115.

[43] A. Pazy: Semigroups of linear operators and applications to partial differential equations. Springer, 1983.

[44] R. S. Phillips: Perturbation theory for semi-groups of linear operators. Trans. Amer. Math. Soc. 74 (1953), 199-221.

[45] M. Reed, B. Simon: Methods of modern mathematical physics I-IV. Academic Press, 1980, 1975, 1979, 1978.

[46] J. Schmid: Adiabatensätze mit und ohne Spektrallückenbedingung. Diplomarbeit Universität Stuttgart. 2010. (Available on arXiv.)

[47] J. Schmid: A note on the well-posedness of non-autonomous linear evolution equations. ArXiv preprint, 2012.

[48] R. Schnaubelt: Well-posedness and asymptotic behaviour of non-autonomous linear evolution equations. Progr. Nonlinear Differential Equations Appl. 50 (2002), 311338.

[49] H. Tanabe: On the equation of evolution in a Banach space. Osaka Math. J. 12 (1960), 363-376.

[50] A. E. Taylor: Introduction to functional analysis. Wiley and Sons, 1958.

[51] A. E. Taylor, D. C. Lay: Introduction to functional analysis. 2nd edition. Wiley, 1980.

[52] S. Teufel: A note on the adiabatic theorem without gap condition. Lett. Math. Phys. 58 (2001), 261-266.

[53] S. Teufel: Adiabatic perturbation theory in quantum dynamics. Lecture Notes in Mathematics 1821. Springer, 2003.

[54] K. Yosida: Functional analysis. 6th edition. Springer, 1980. 